\title{
Regioselective Activation of Oxo Ligands in Functionalized Dawson Polyoxotungstates
}

\author{
Cécile Boglio, Kévin Micoine, Étienne Derat, René Thouvenot, Bernold Hasenknopf, Serge Thorimbert, Emmanuel \\ Lacôte, Max Malacria
}

\section{Supporting information}

General remarks: Reagents and chemicals were purchased from commercial sources and used as received. Functionnalized polyoxometalates $\mathrm{TBA}_{6} \mathrm{H}\left[\alpha_{1}-\mathrm{P}_{2} \mathrm{~W}_{17} \mathrm{O}_{61}\left\{\mathrm{SnCH}_{2} \mathrm{CH}_{2} \mathrm{CO}_{2} \mathrm{H}\right\}\right]$ (1) and $\mathrm{TBA}_{6} \mathrm{~K}\left[\alpha_{2^{-}}\right.$ $\left.\mathrm{P}_{2} \mathrm{~W}_{17} \mathrm{O}_{61}\left\{\mathrm{SnCH}_{2} \mathrm{CH}_{2} \mathrm{CO}_{2} \mathrm{H}\right\}\right]$ (2) were prepared following methods reported in the literature (see Ref. 1). Unless otherwise noted, reactions were carried out under air atmosphere with magnetic stirring. $\mathrm{CH}_{3} \mathrm{CN}$ was dried and distilled from $\mathrm{CaH}_{2}$. Thin-layer chromatography (TLC) was performed on Merck $60 \mathrm{~F}_{254}$ silica gel. IR spectra were recorded from a Bruker Tensor 27 ATR diamond PIKE spectrophotometer. ${ }^{1} \mathrm{H}$ NMR $\left[{ }^{13} \mathrm{C}\right.$ NMR] spectra were recorded at room temperature with a $400 \mathrm{MHz}$ [100 MHz] Bruker AVANCE 400 spectrometer or a $200 \mathrm{MHz}$ [50 MHz] Bruker AVANCE 200. Chemical shifts are given in ppm, referenced to TMS $(\delta=0 \mathrm{ppm})$ using the solvent signals $\delta=1.94 \mathrm{ppm}$ for $\mathrm{CHD}_{2} \mathrm{CN}\left[\delta=118.3 \mathrm{ppm}\right.$ for $\mathrm{CD}_{3} \mathrm{CN}$ ].Coupling constants $(J)$ are given in Hertz $(\mathrm{Hz}) .{ }^{31} \mathrm{P}$ NMR spectra were obtained at $298 \mathrm{~K}$ in $5 \mathrm{~mm}$ o.d. tube at $162 \mathrm{MHz}$, on a Bruker AVANCE 400 spectrometer at a concentration of $100 \mathrm{mg} / 0.5 \mathrm{~mL}$. External $85 \% \mathrm{H}_{3} \mathrm{PO}_{4}$ in coaxial tube was used as reference. ${ }^{183} \mathrm{~W}$ NMR spectra $(300 \mathrm{~K}$ ) were recorded in $10 \mathrm{~mm}$ o.d. tubes (sample volume $2.5 \mathrm{~mL}$ ) at $12.5 \mathrm{MHz}$ on a Bruker Avance II 300 equipped with a low-frequency special VSP probehead, and at $20.8 \mathrm{MHz}$ on a Bruker DRX500 spectrometer with a standard tunable BBO probehead. Chemical shifts are referenced to $\mathrm{WO}_{4}{ }^{2-}(\delta=0 \mathrm{ppm})$. They were measured by the substitution method, using a saturated aqueous solution (in $\left.\mathrm{D}_{2} \mathrm{O}\right)$ of dodecatungstosilicic acid $\left(\mathrm{H}_{4} \mathrm{SiW}_{12} \mathrm{O}_{40}\right)$ as a secondary standard $(\delta=$ $-103.8 \mathrm{ppm}) .{ }^{119} \mathrm{Sn}$ NMR spectra $(300 \mathrm{~K})$ were recorded in $10 \mathrm{~mm}$ o.d. tubes at $111.9 \mathrm{MHz}$ on a Bruker Avance II 300 equipped with a standard tunable BBO probehead. Chemical shifts are referenced to $\left(\mathrm{CH}_{3}\right)_{4} \mathrm{Sn}$ and were measured by the substitution method. Mass spectrometry experiments have been carried out on an electrospray-ion trap instrument (Bruker, Esquire 3000). The $50 \mu \mathrm{mol} \cdot \mathrm{L}^{-1}$ solutions of POMs were infused using a syringe pump $\left(160 \mu \mathrm{L} \cdot \mathrm{h}^{-1}\right)$. The negative ion mode was used with capillary high voltage $3500 \mathrm{~V}$. The orifice/skimmer voltage difference was set to $45 \mathrm{~V}$ to avoid decomposition of the POMs. The low-mass-cutoff (LMCO) of the ion trap was set to $80 \mathrm{Th} .(\mathrm{K}),\left(\alpha_{1}\right),\left(\alpha_{2}\right)$ represent respectively the formulas of lacunary Keggin polyanion $\left(\left[\mathrm{PW}_{11} \mathrm{O}_{39}\right]^{7-}\right)$, lacunary Dawson polyanions $\left(\left[\mathrm{P}_{2} \mathrm{~W}_{17} \mathrm{O}_{61}\right]^{10-}, \alpha_{1}\right.$ and $\alpha_{2}$ isomers). Elemental analyses were carried out by the "Service Central d'Analyse", CNRS, Vernaison, France or by the ICSN, CNRS, Gif, France.

\section{General procedure for amide formation (GPA).}

To a solution of $\mathrm{TBA}_{6} \mathrm{H}\left[\mathrm{P}_{2} \mathrm{~W}_{17} \mathrm{O}_{61}\left\{\mathrm{SnCH}_{2} \mathrm{CH}_{2} \mathrm{C}(=\mathrm{O})\right](\mathbf{2}\right.$ or $\mathbf{4}, 0.026 \mathrm{mmol}, 150 \mathrm{mg})$, in acetonitrile $(3 \mathrm{~mL})$ was added the amine $(0.052 \mathrm{mmol}, 2$ equiv) and triethylamine $(0.031 \mathrm{mmol}, 1.2$ equiv). The mixture was stirred at rt for 4 hours. A cation-exchange resin (Amberlyst 15, 16-50 mesh, $\mathrm{TBA}^{+}$form) was added, followed by acetone $(10 \mathrm{~mL})$ and the mixture was stirred for 1 hour. The resin was filtered off and the filtrate was concentrated in vacuo. The oil which was obtained was dissolved in acetone $(2 \mathrm{~mL})$, and precipitated upon addition of $\mathrm{EtOH} / \mathrm{Et}_{2} \mathrm{O}(2 \mathrm{~mL} / 30 \mathrm{~mL})$. The solid was isolated by centrifugation, washed with $\mathrm{Et}_{2} \mathrm{O}$ and dried in vacuo to afford the desired POM.

\section{General procedure for thioester formation (GPB).}

To a solution of $\mathrm{TBA}_{6}\left[\mathrm{P}_{2} \mathrm{~W}_{17} \mathrm{O}_{61}\left\{\mathrm{SnCH}_{2} \mathrm{CH}_{2} \mathrm{C}(=\mathrm{O})\right](\mathbf{2}\right.$ or $\mathbf{4}, 0.041 \mathrm{mmol}, 200 \mathrm{mg})$, in acetonitrile $(3 \mathrm{~mL})$ was added the thiol $(0.068 \mathrm{mmol}, 2$ equiv), triethylamine $(0.034 \mathrm{mmol}, 1.2$ equiv) and dimethylaminopyridine ( $0.017 \mathrm{mmol}, 0.5$ equiv). The mixture was stirred at $\mathrm{rt}$ for 4 hours. A cation-exchange resin (Amberlyst 15, $16-50$ mesh, $\mathrm{TBA}^{+}$form) was added, followed by acetone $(10 \mathrm{~mL})$ and the mixture was stirred for 1 hour. The resin was filtered off and the filtrate was concentrated in vacuo. The oil which was obtained was dissolved in acetone $(2 \mathrm{~mL})$, and precipitated upon addition of $\mathrm{EtOH} / \mathrm{Et}_{2} \mathrm{O}(2 \mathrm{~mL} / 30 \mathrm{~mL})$. The solid was isolated by centrifugation, washed with $\mathrm{Et}_{2} \mathrm{O}$ and dried in vacuo to afford the desired $\mathrm{POM}$. 
2: $\mathrm{TBA}_{6}\left[\alpha_{1}-\mathrm{P}_{2} \mathrm{~W}_{17} \mathrm{O}_{61}\left[\mathrm{SnCH}_{2} \mathrm{CH}_{2} \mathrm{C}(=\mathrm{O})\right]\right.$. ESI $/ \mathrm{MS}$ :

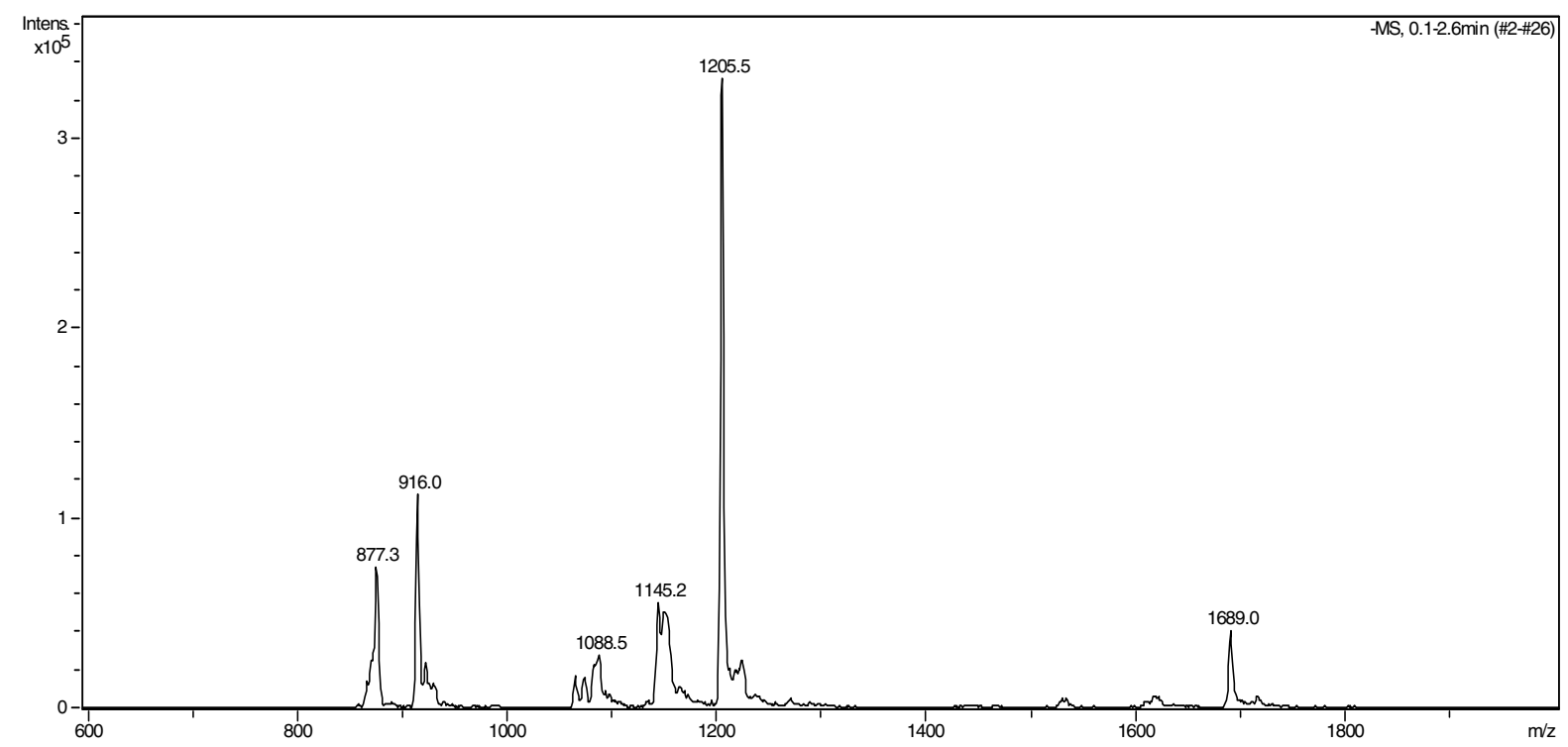

\begin{tabular}{cccccc}
\hline Entry & Charge & $\begin{array}{c}\text { Simulated } \\
\mathrm{m} / \mathrm{z}\end{array}$ & $\begin{array}{c}\text { Observed } \\
\mathrm{m} / \mathrm{z}\end{array}$ & $\begin{array}{c}\text { Relative } \\
\text { intensity }\end{array}$ & Composition $^{[\mathrm{a}]}$ \\
\hline 1 & $3-$ & 1688.9 & 1689.0 & 12 & $\mathrm{TBA}_{3} \mathrm{H}\left[\left(\alpha_{1}\right) \mathrm{Sn}\left(\mathrm{CH}_{2}\right)_{2} \mathrm{CO}\right]$ \\
2 & $4-$ & 1205.8 & 1205.5 & 100 & $\mathrm{TBA}_{2} \mathrm{H}\left[\left(\alpha_{1}\right) \mathrm{Sn}\left(\mathrm{CH}_{2}\right)_{2} \mathrm{CO}\right]$ \\
3 & $4-$ & 1145.5 & 1145.2 & 17 & $\mathrm{TBAH}_{2}\left[\left(\alpha_{1}\right) \mathrm{Sn}\left(\mathrm{CH}_{2}\right)_{2} \mathrm{CO}\right]$ \\
4 & $4-$ & 1085.2 & 1084.7 & 7 & $\mathrm{H}_{3}\left[\left(\alpha_{1}\right) \mathrm{Sn}\left(\mathrm{CH}_{2}\right)_{2} \mathrm{CO}\right]$ \\
5 & $5-$ & 916.2 & 916.0 & 34 & $\left.\mathrm{TBAH}_{(}\left(\alpha_{1}\right) \mathrm{Sn}\left(\mathrm{CH}_{2}\right)_{2} \mathrm{CO}\right]$ \\
6 & $5-$ & 867.7 & 877.3 & 23 & $\mathrm{H}_{2}\left[\left(\alpha_{1}\right) \mathrm{Sn}\left(\mathrm{CH}_{2}\right)_{2} \mathrm{CO}\right]$ \\
\hline
\end{tabular}

[a] $\left(\alpha_{1}\right)=\alpha_{1}-\mathrm{P}_{2} \mathrm{~W}_{17} \mathrm{O}_{61}$

\section{4: $\mathrm{TBA}_{6}\left[\alpha_{2}-\mathrm{P}_{2} \mathrm{~W}_{17} \mathrm{O}_{61}\left[\mathrm{SnCH}_{2} \mathrm{CH}_{2} \mathrm{C}(=\mathrm{O})\right]\right.$. ESI $/ \mathrm{MS}$ :}

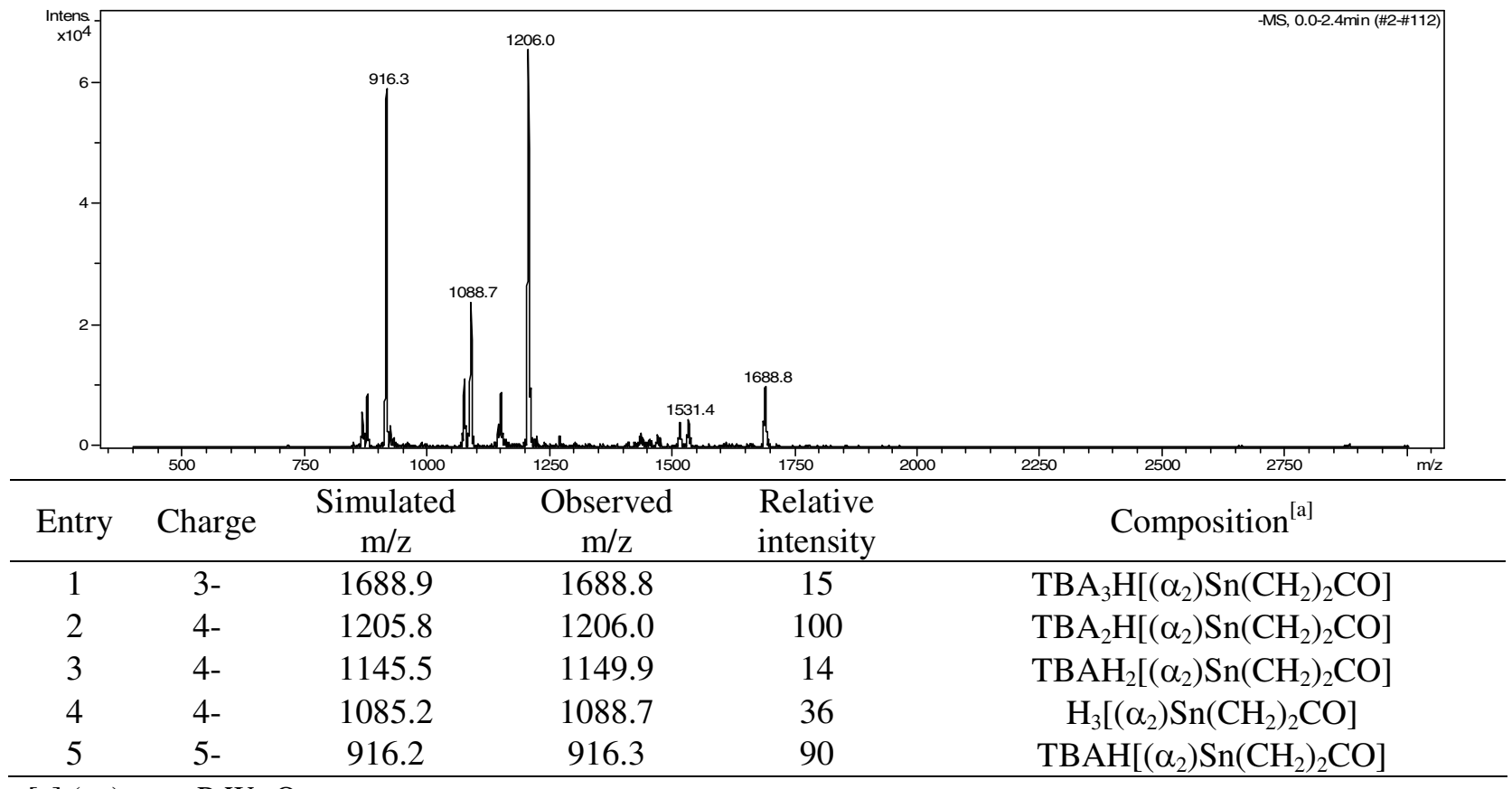

$[\mathrm{a}]\left(\alpha_{2}\right)=\alpha_{1}-\mathrm{P}_{2} \mathrm{~W}_{17} \mathrm{O}_{61}$ 


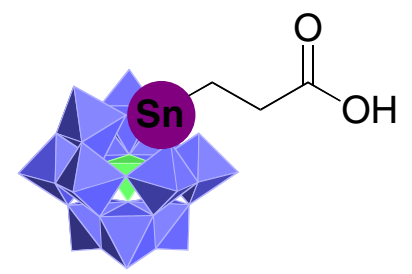

5: $\mathbf{T B A}_{4}\left[\boldsymbol{\alpha}-\mathbf{P W}_{11} \mathbf{O}_{39} \mathbf{S n C H}_{2} \mathbf{C H}_{2} \mathbf{C O}_{2} \mathbf{H}\right]$. TBABr (4.0 mmol, 4 equiv., $1.33 \mathrm{~g}$ ) was added to a suspension of lacunary $\mathrm{K}_{7}\left[\alpha-\mathrm{PW}_{11} \mathrm{O}_{39}\right](1.0 \mathrm{mmol}, 3.0 \mathrm{~g})$ in acetonitrile $(50 \mathrm{~mL})$. Then $\mathrm{Cl}_{3}\left(\mathrm{CH}_{2}\right)_{2} \mathrm{COOH}(2.0 \mathrm{mmol}, 2$ equiv., $596 \mathrm{mg}$ ) were added, and the mixture was stirred at room temperature for 2 or 3 hours under an argon atmosphere. The remaining solid was filtred off and the filtrate was concentrated under vacuo. The white powder obtained was solubilized in acetone $(5 \mathrm{~mL})$ and precipited by adding $\mathrm{EtOH} / \mathrm{Et}_{2} \mathrm{O}(5 \mathrm{~mL} / 100 \mathrm{~mL})$ to afford the desired substituted POM 5 (0.80 mmol, $3.06 \mathrm{~g}, 80 \%$ yield). IR: $\tilde{v}=2961(\mathrm{~m}), 2935(\mathrm{w}), 2873(\mathrm{~m})$, 1727 (w), 1483 (m), 1380 (w), 1153 (vw), 1064 (s), 959 (s), 881 (s), 791 (vs) cm ${ }^{-1}$; ${ }^{1} \mathrm{H}$ NMR (400 MHz, $\left.\mathrm{CD}_{3} \mathrm{CN}\right): \delta$ 0.94-1.03 (m, $\left.48 \mathrm{H}, \mathrm{N}\left(\mathrm{CH}_{2} \mathrm{CH}_{2} \mathrm{CH}_{2} \mathrm{Me}\right)_{4}\right), 1.30-1.48\left(\mathrm{~m}, 34 \mathrm{H}, \mathrm{N}\left(\mathrm{CH}_{2} \mathrm{CH}_{2} \mathrm{CH}_{2} \mathrm{Me}\right)_{4}+\mathrm{SnCH}_{2}\right)$, 1.55-1.69 (m, $\left.32 \mathrm{H}, \mathrm{N}\left(\mathrm{CH}_{2} \mathrm{CH}_{2} \mathrm{CH}_{2} \mathrm{CH}_{3}\right)_{4}\right), 2.62\left(\mathrm{dd}, J=8.6\right.$ and $\left.7.1 \mathrm{~Hz}, 2 \mathrm{H}, \mathrm{CH}_{2} \mathrm{C}=\mathrm{O}\right), 3.12-3.20(\mathrm{~m}, 32 \mathrm{H}$,

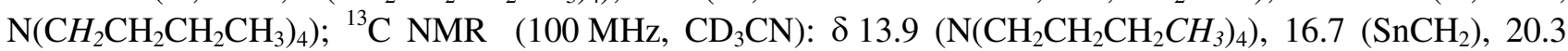

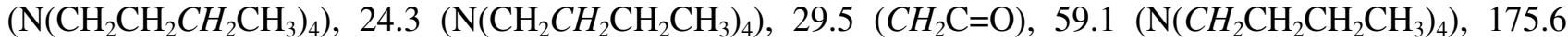
$(\mathrm{C}=\mathrm{O}) ;{ }^{31} \mathrm{P}$ NMR $\left(162 \mathrm{MHz}, \mathrm{CD}_{3} \mathrm{CN}\right): \delta-10,90\left(\mathrm{~s}+\mathrm{d}, 1 \mathrm{P}, J_{S n P}=19.8 \mathrm{~Hz}\right)$.

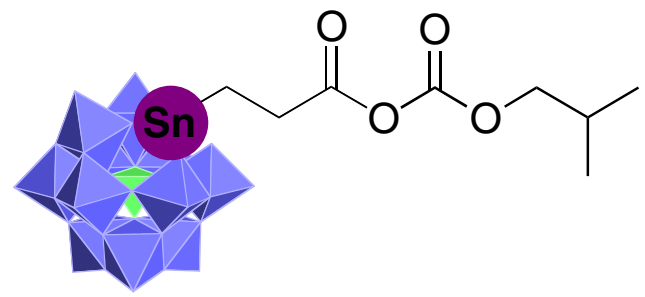

6: $\mathbf{T B A}_{4}\left[\boldsymbol{\alpha}-\mathbf{P W}_{11} \mathbf{O}_{39} \mathrm{SnCH}_{2} \mathrm{CH}_{2} \mathrm{CO}_{2} \mathrm{CO}_{2}{ }^{\mathrm{i}} \mathrm{Bu}\right] . \mathrm{NEt}_{3}(0.143 \mathrm{mmol}, 1.1$ equiv., $20 \mu \mathrm{L})$ was added to a solution of $\mathrm{TBA}_{4}\left[(\mathrm{~K}) \mathrm{SnCH}_{2} \mathrm{CH}_{2} \mathrm{CO}_{2} \mathrm{H}\right] \mathbf{5}(0.13 \mathrm{mmol}, 500 \mathrm{mg})$ in acetonitrile $(10 \mathrm{~mL})$. After $5 \mathrm{~min}$, isobutylchloroformiate $(0.156 \mathrm{mmol}, 1.5$ equiv., $20 \mu \mathrm{L})$ was added, and the mixture was stirred at room temperature for 45 min. under an argon atmosphere. A cation-exchange resin (Amberlyst 15, 16-50 mesh, $\mathrm{TBA}^{+}$form) was added, followed by acetone $(10 \mathrm{~mL})$ and the mixture was stirred for 1 hour. The resin was filtered off and the filtrate was concentrated in vacuo. The oil which was obtained was dissolved in acetone $(3 \mathrm{~mL})$, and precipitated upon addition of $\mathrm{CH}_{2} \mathrm{Cl}_{2} / \mathrm{Et}_{2} \mathrm{O}(3 \mathrm{~mL} / 50 \mathrm{~mL})$. The solid was isolated by filtration, washed with $\mathrm{Et}_{2} \mathrm{O}$ and dried in vacuo to afford the desired POM 6 as a white powder $(0.127 \mathrm{mmol}, 502 \mathrm{mg}, 98 \%$ yield $)$. IR: $\tilde{v}=2960(\mathrm{~m}), 2934(\mathrm{w}), 2873(\mathrm{w}), 1725(\mathrm{w}), 1482(\mathrm{~m}), 1381(\mathrm{w}), 1152(\mathrm{~m}), 1064(\mathrm{~s}), 959(\mathrm{~s}), 881(\mathrm{~s})$, 790 (vs) cm ${ }^{-1} ;{ }^{1} \mathrm{H}$ NMR (400 MHz, $\mathrm{CD}_{3} \mathrm{CN}$ ): $\delta 0.93$ (d, $J=6.5 \mathrm{~Hz}, 6 \mathrm{H}, \mathrm{CHMe}$ ), $0.98(\mathrm{t}, J=7.3 \mathrm{~Hz}, 48 \mathrm{H}$, $\left.\mathrm{N}\left(\mathrm{CH}_{2} \mathrm{CH}_{2} \mathrm{CH}_{2} \mathrm{Me}\right)_{4}\right), \quad 1.34-1.47\left(\mathrm{~m}, 43 \mathrm{H}, \quad \mathrm{N}\left(\mathrm{CH}_{2} \mathrm{CH}_{2} \mathrm{CH}_{2} \mathrm{Me}\right)_{4}+\mathrm{SnCH}_{2}\right), \quad 1.61-1.71 \quad(\mathrm{~m}, \quad 32 \mathrm{H}$, $\left.\mathrm{N}\left(\mathrm{CH}_{2} \mathrm{CH}_{2} \mathrm{CH}_{2} \mathrm{Me}\right)_{4}\right), 1.93-2.03\left(\mathrm{~m}, 1 \mathrm{H}, \mathrm{CH}_{2} \mathrm{CHMe}\right.$ ), $2.81\left(\mathrm{t}, J=8.6 \mathrm{~Hz}, 2 \mathrm{H}, \mathrm{CH}_{2} \mathrm{C}=\mathrm{O}\right), 3.09-3.26(\mathrm{~m}, 32$ $\left.\mathrm{H}, \mathrm{N}\left(\mathrm{CH}_{2} \mathrm{CH}_{2} \mathrm{CH}_{2} \mathrm{Me}\right)_{4}\right), 4.00\left(\mathrm{~d}, J=6.8 \mathrm{~Hz}, 2 \mathrm{H}, \mathrm{OCH}_{2} \mathrm{CH}\right) ;{ }^{13} \mathrm{C} \mathrm{NMR}\left(100 \mathrm{MHz}, \mathrm{CD}_{3} \mathrm{CN}\right): \delta 14.0$

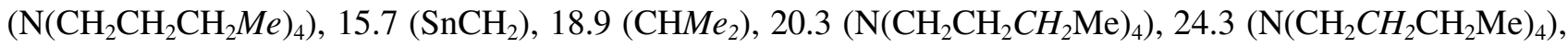
$28.3\left(\mathrm{CH}_{2} \mathrm{CHMe}\right), 30.2\left(\mathrm{CH}_{2} \mathrm{C}=\mathrm{O}\right), 59.1\left(\mathrm{~N}\left(\mathrm{CH}_{2} \mathrm{CH}_{2} \mathrm{CH}_{2} \mathrm{Me}\right)_{4}\right), 75.9\left(\mathrm{OCH}_{2} \mathrm{CH}\right), 150.0(\mathrm{OC}(=\mathrm{O}) \mathrm{O}), 170.1$ $\left(\mathrm{CH}_{2} \mathrm{C}(=\mathrm{O}) \mathrm{O}\right) ;{ }^{31} \mathrm{P} \mathrm{NMR}\left(162 \mathrm{MHz}, \mathrm{CD}_{3} \mathrm{CN}\right): \delta-10.89\left(\mathrm{~s}+\mathrm{d}, 1 \mathrm{P}, J_{S n P}=19.8 \mathrm{~Hz}\right)$. 


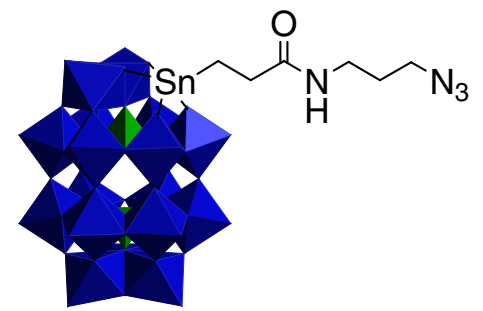

8a: $\mathbf{T B A}_{7}\left[\alpha_{2}-\mathbf{P}_{2} \mathbf{W}_{17} \mathbf{O}_{61} \mathbf{S n C H}_{2} \mathbf{C H}_{2} \mathbf{C}(=\mathbf{O}) \mathbf{N H}\left(\mathbf{C H}_{2}\right)_{3} \mathbf{N}_{3}\right]$. Following GPA, 8a was obtained in $88 \%$ yield. IR: $\tilde{v}=2961(\mathrm{~m}), 2934(\mathrm{w}), 2873(\mathrm{~m}), 2095(\mathrm{~m}), 1634(\mathrm{w}), 1482(\mathrm{~m}), 1379(\mathrm{w}), 1086(\mathrm{~s}), 945(\mathrm{~s}), 898(\mathrm{~s}), 764$ (vs) $\mathrm{cm}^{-1} ;{ }^{1} \mathrm{H}$ NMR $\left(400 \mathrm{MHz}, \mathrm{CD}_{3} \mathrm{CN}+5 \% \mathrm{D}_{2} \mathrm{O}\right): \delta 0.98\left(\mathrm{t}, J=7.1 \mathrm{~Hz}, 81 \mathrm{H}, \mathrm{N}\left[\left(\mathrm{CH}_{2}\right)_{3} M e\right]_{4}\right), 1.21(\mathrm{t}$, $\left.J=8.0 \mathrm{~Hz}, \quad 2 \mathrm{H}, \quad \mathrm{SnCH}_{2}\right), \quad 1.38-1.44 \quad\left(\mathrm{~m}, \quad 54 \quad \mathrm{H}, \quad \mathrm{N}\left(\mathrm{CH}_{2} \mathrm{CH}_{2} \mathrm{CH}_{2} \mathrm{Me}\right)_{4}\right), \quad 1.55-1.70 \quad(\mathrm{~m}, \quad 54 \mathrm{H}$, $\left.\mathrm{N}\left(\mathrm{CH}_{2} \mathrm{CH}_{2} \mathrm{CH}_{2} \mathrm{Me}\right)_{4}\right), 1.72\left(\mathrm{~m}, 2 \mathrm{H}, \mathrm{CH}_{2} \mathrm{CH}_{2} \mathrm{CH}_{2}\right), 2.49\left(\mathrm{t}, J=8.0 \mathrm{~Hz}, 2 \mathrm{H}, \mathrm{CH}_{2} \mathrm{C}=\mathrm{O}\right), 3.08-3.25(\mathrm{~m}, 56 \mathrm{H}$, $\left.\mathrm{N}\left(\mathrm{CH}_{2} \mathrm{CH}_{2} \mathrm{CH}_{2} \mathrm{Me}\right)_{4}+\mathrm{NHCH}_{2}\right), 3.34\left(\mathrm{t}, J=6.8 \mathrm{~Hz}, 2 \mathrm{H}, \mathrm{CH}_{2} \mathrm{~N}_{3}\right) ;{ }^{13} \mathrm{C} \mathrm{NMR}\left(50 \mathrm{MHz}, \mathrm{CD}_{3} \mathrm{CN}+5 \% \mathrm{D}_{2} \mathrm{O}\right): \delta$ $\left.13.4 \quad\left(\mathrm{~N}^{2} \mathrm{CH}_{2}\right]_{3} \mathrm{Me}\right), \quad 18.5 \quad\left(\mathrm{SnCH}_{2}\right), \quad 19.8 \quad\left(\mathrm{~N}\left(\mathrm{CH}_{2} \mathrm{CH}_{2} \mathrm{CH}_{2} \mathrm{Me}\right)_{4}\right), \quad 23.8 \quad\left(\mathrm{~N}\left(\mathrm{CH}_{2} \mathrm{CH}_{2} \mathrm{CH}_{2} \mathrm{Me}\right)_{4}\right), \quad 28.6$ $\left(\mathrm{CH}_{2} \mathrm{CH}_{2} \mathrm{CH}_{2}\right), 31.3\left(\mathrm{CH}_{2} \mathrm{C}=\mathrm{O}\right), 36.9\left(\mathrm{NHCH}_{2}\right), 49.3\left(\mathrm{CH}_{2} \mathrm{~N}_{3}\right), 58.6\left(\mathrm{~N}\left(\mathrm{CH}_{2} \mathrm{Pr}\right)_{4}\right), 176.1(\mathrm{C}=\mathrm{O}) ;{ }^{31} \mathrm{P}$ NMR $\left(162 \mathrm{MHz}, \mathrm{CD}_{3} \mathrm{CN}+5 \% \mathrm{D}_{2} \mathrm{O}\right): \delta-9.5\left(\mathrm{~s}+\mathrm{d}, 1 \mathrm{P}, J_{S n P}=22.7 \mathrm{~Hz}\right),-12.2(\mathrm{~s}, 1 \mathrm{P})$.

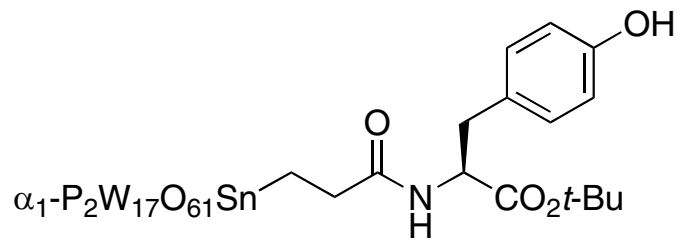

8b: $\mathrm{TBA}_{7}\left[\alpha_{1}-\mathrm{P}_{2} \mathrm{~W}_{17} \mathrm{O}_{61}\left\{\mathrm{SnCH}_{2} \mathrm{CH}_{2} \mathrm{CONHCH}\left(\mathrm{CH}_{2} \mathrm{p}-\mathrm{C}_{6} \mathrm{H}_{4} \mathrm{OH}\right) \mathrm{CO}_{2}{ }^{\mathrm{t} B u}\right\}\right]$. Following GPA, 8b was obtained in $86 \%$ yield. Spectral data corresponded to those described in the literature ${ }^{1}$; ESI/MS: see below for full details.

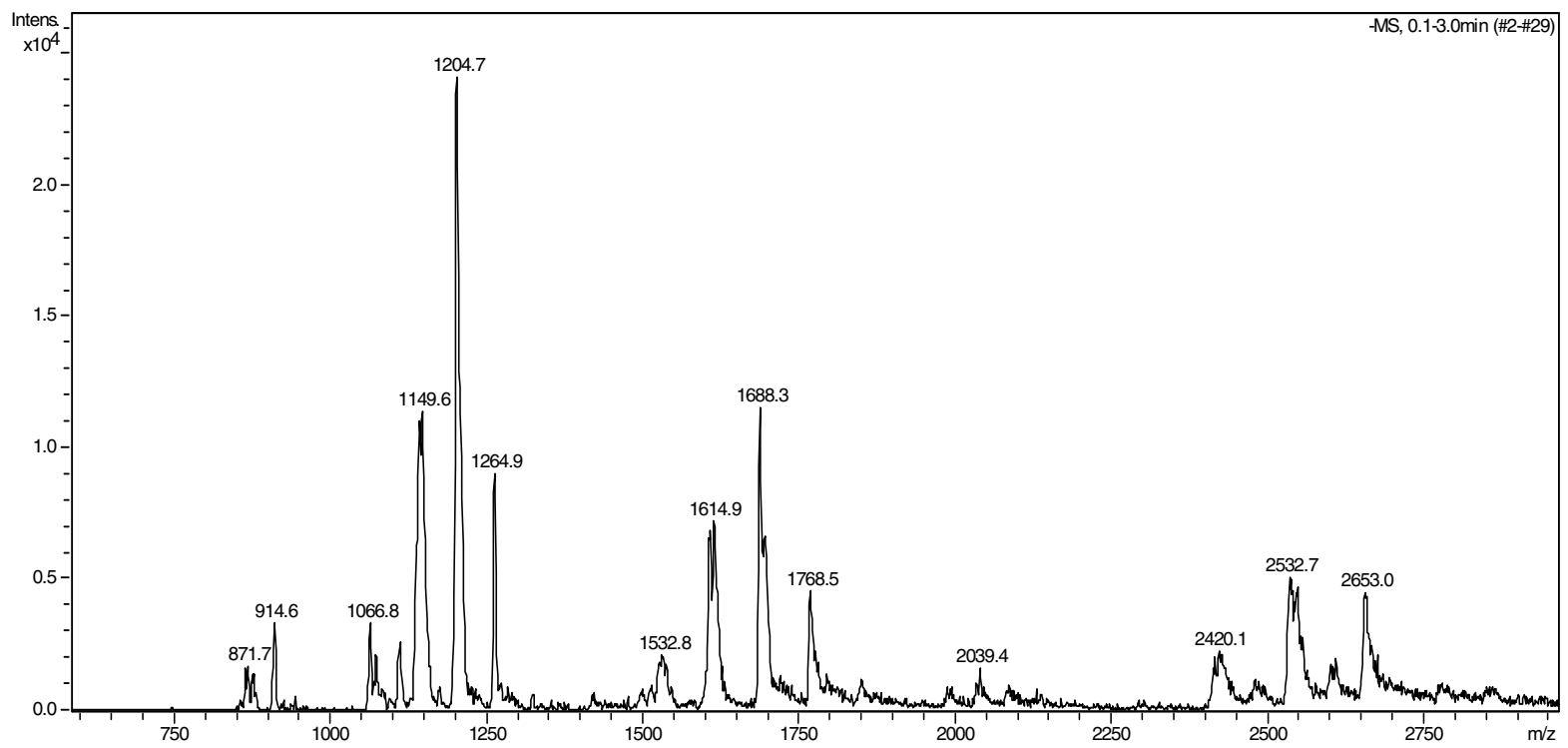

\footnotetext{
${ }^{1}$ Bareyt, S.; Piligkos, S.; Hasenknopf, B.; Gouzerh, P.; Lacôte, E.; Thorimbert, S.; Malacria, M. J. Am. Chem. Soc. 2005, 127, 6788-6794.
} 


\begin{tabular}{cccccc}
\hline Entry & Charge & $\begin{array}{c}\text { Simulated } \\
\mathrm{m} / \mathrm{z}\end{array}$ & $\begin{array}{c}\text { Observed } \\
\mathrm{m} / \mathrm{z}\end{array}$ & $\begin{array}{c}\text { Relative } \\
\text { intensity }\end{array}$ & Composition \\
\hline 1 & $2-$ & 2651.9 & 2653.5 & 18 & $\left(n \mathrm{Bu}_{4} \mathrm{~N}\right)_{3} \mathrm{H}_{2}\left[\left(\alpha_{1}\right) \mathrm{Sn}\left(\mathrm{CH}_{2}\right)_{2} \mathrm{CO}_{2} \mathrm{R}\right]$ \\
2 & $2-$ & 2531.2 & 2533.4 & 21 & $\left(n \mathrm{Bu}_{4} \mathrm{~N}\right)_{2} \mathrm{H}_{3}\left[\left(\alpha_{1}\right) \mathrm{Sn}\left(\mathrm{CH}_{2}\right)_{2} \mathrm{CO}_{2} \mathrm{R}\right]$ \\
3 & $2-$ & 2410.1 & 2420.2 & 9 & $\left(n \mathrm{Bu}_{4} \mathrm{~N}^{2} \mathrm{H}_{4}\left[\left(\alpha_{1}\right) \mathrm{Sn}\left(\mathrm{CH}_{2}\right)_{2} \mathrm{CO}_{2} \mathrm{R}\right]+1 \mathrm{H}_{2} \mathrm{O}\right.$ \\
4 & $3-$ & 1767.6 & 1768.4 & 7 & $\left(n \mathrm{Bu}_{4} \mathrm{~N}\right)_{3} \mathrm{H}\left[\left(\alpha_{1}\right) \mathrm{Sn}\left(\mathrm{CH}_{2}\right)_{2} \mathrm{CO}_{2} \mathrm{R}\right]$ \\
5 & $3-$ & 1687.2 & 1688.5 & 48 & $\left(n \mathrm{Bu}_{4} \mathrm{~N}\right)_{2} \mathrm{H}_{2}\left[\left(\alpha_{1}\right) \mathrm{Sn}\left(\mathrm{CH}_{2}\right)_{2} \mathrm{CO}_{2} \mathrm{R}\right]$ \\
6 & $3-$ & 1606.4 & 1614.9 & 29 & $\left(n \mathrm{Bu}_{4} \mathrm{~N}_{3} \mathrm{H}_{3}\left[\left(\alpha_{1}\right) \mathrm{Sn}\left(\mathrm{CH}_{2}\right)_{2} \mathrm{CO}_{2} \mathrm{R}\right]+1 \mathrm{H}_{2} \mathrm{O}\right.$ \\
7 & $3-$ & 1606.4 & 1608.2 & 28 & $\left(n \mathrm{Bu}_{4} \mathrm{~N}\right) \mathrm{H}_{3}\left[\left(\alpha_{1}\right) \mathrm{Sn}\left(\mathrm{CH}_{2}\right)_{2} \mathrm{CO}_{2} \mathrm{R}\right]$ \\
8 & $4-$ & 1265.1 & 1265.0 & 38 & $\left(n \mathrm{Bu}_{4} \mathrm{~N}\right)_{2} \mathrm{H}\left[\left(\alpha_{1}\right) \mathrm{Sn}\left(\mathrm{CH}_{2}\right)_{2} \mathrm{CO}_{2} \mathrm{R}\right]$ \\
9 & $4-$ & 1204.5 & 1204.9 & 100 & $\left(n \mathrm{Bu}_{4} \mathrm{~N}\right) \mathrm{H}_{2}\left[\left(\alpha_{1}\right) \mathrm{Sn}\left(\mathrm{CH}_{2}\right)_{2} \mathrm{CO}_{2} \mathrm{R}\right]$ \\
10 & $4-$ & 1144.2 & 1149.6 & 47 & $\mathrm{H}_{3}\left[\left(\alpha_{1}\right) \mathrm{Sn}\left(\mathrm{CH}_{2}\right)_{2} \mathrm{CO}_{2} \mathrm{R}_{+}+1 \mathrm{H}_{2} \mathrm{O}\right.$ \\
11 & $4-$ & 1144.2 & 1145.0 & 44 & $\mathrm{H}_{3}\left[\left(\alpha_{1}\right) \mathrm{Sn}\left(\mathrm{CH}_{2}\right)_{2} \mathrm{CO}_{2} \mathrm{R}\right]$ \\
12 & $5-$ & 915.2 & 914.5 & 13 & $\mathrm{H}_{2}\left[\left(\alpha_{1}\right) \mathrm{Sn}\left(\mathrm{CH}_{2}\right)_{2} \mathrm{CO}_{2} \mathrm{R}\right]$ \\
\hline
\end{tabular}

$\mathrm{R}=\mathrm{L}-\mathrm{tyr}-\mathrm{OtBu}$<smiles></smiles>

8c: $\mathbf{T B A}_{7}\left[\alpha_{1}-\mathbf{P}_{2} \mathbf{W}_{17} \mathbf{O}_{61}\left\{\mathbf{S n C H}_{2} \mathbf{C H}_{2} \mathbf{C O N H C H}(\mathbf{P h})-\mathbf{C H}(\mathbf{P h}) \mathbf{N H}_{2}\right\}\right]$. Following GPA, 8c was obtained in 94\% yield. Spectral data corresponded to those described in the literature ${ }^{1}$; ESI/MS: see below for full details.

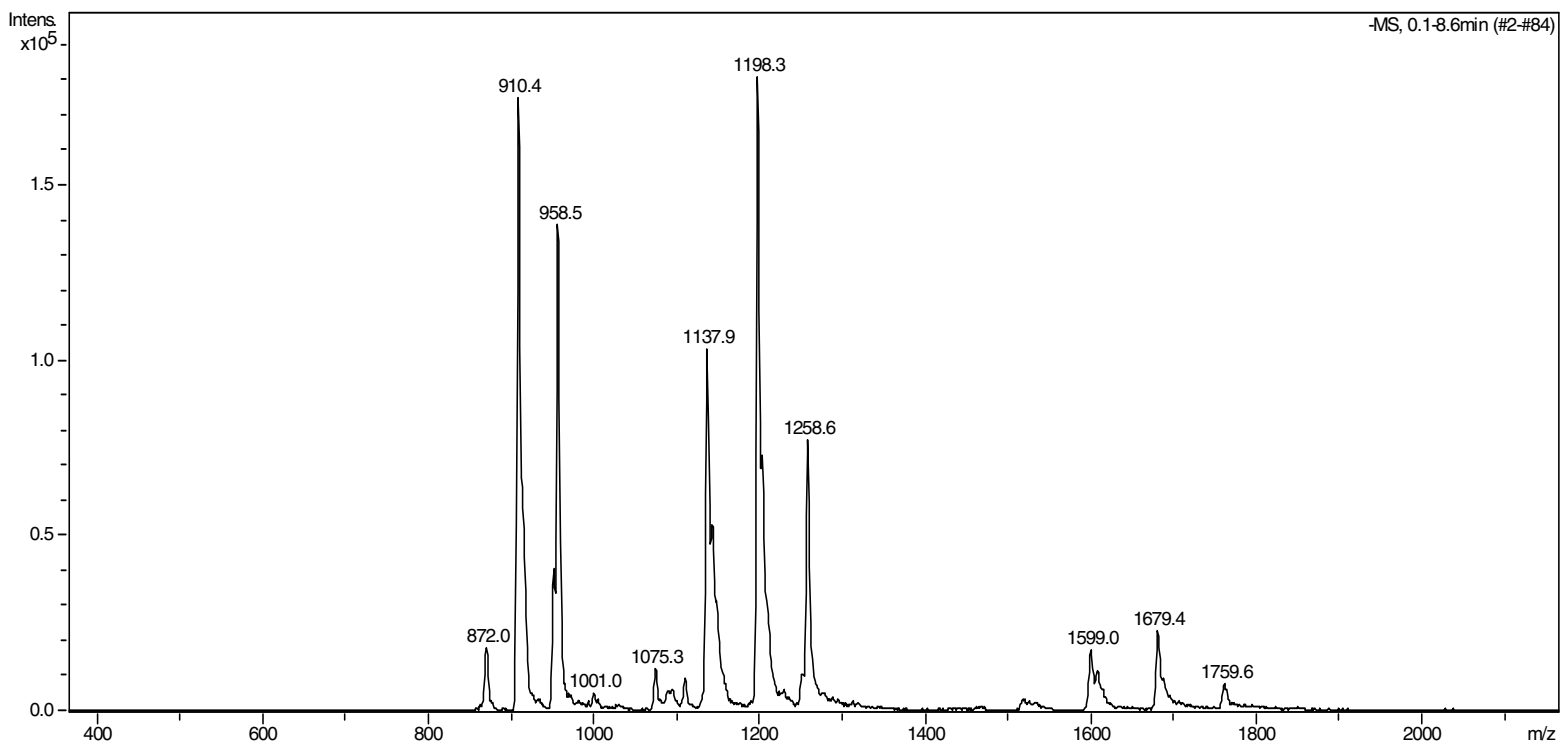

\footnotetext{
${ }^{1}$ Bareyt, S.; Piligkos, S.; Hasenknopf, B.; Gouzerh, P.; Lacôte, E.; Thorimbert, S.; Malacria, M. J. Am. Chem. Soc. 2005, 127, 6788-6794.
} 


\begin{tabular}{cccccc}
\hline Entry & Charge & $\begin{array}{c}\text { Simulated } \\
\mathrm{m} / \mathrm{z}\end{array}$ & $\begin{array}{c}\text { Observed } \\
\mathrm{m} / \mathrm{z}\end{array}$ & $\begin{array}{c}\text { Relative } \\
\text { intensity }\end{array}$ & Composition \\
\hline 1 & $3-$ & 1759.2 & 1759.8 & 4 & $\left(n \mathrm{Bu}_{4} \mathrm{~N}\right)_{3} \mathrm{H}\left[\left(\alpha_{1}\right) \mathrm{Sn}\left(\mathrm{CH}_{2}\right)_{2} \mathrm{CO}_{2} \mathrm{R}\right]$ \\
2 & $3-$ & 1678.8 & 1679.4 & 13 & $\left(n \mathrm{Bu}_{4} \mathrm{~N}\right)_{2} \mathrm{H}_{2}\left[\left(\alpha_{1}\right) \mathrm{Sn}\left(\mathrm{CH}_{2}\right)_{2} \mathrm{CO}_{2} \mathrm{R}\right]$ \\
3 & $3-$ & 1598.1 & 1598.9 & 10 & $\left(n \mathrm{Bu}_{4} \mathrm{~N}\right) \mathrm{H}_{3}\left[\left(\alpha_{1}\right) \mathrm{Sn}\left(\mathrm{CH}_{2}\right)_{2} \mathrm{CO}_{2} \mathrm{R}\right]$ \\
4 & $3-$ & 1517.6 & 1518.2 & 2 & $\mathrm{H}_{4}\left[\left(\alpha_{1}\right) \mathrm{Sn}\left(\mathrm{CH}_{2}\right)_{2} \mathrm{CO}_{2} \mathrm{R}\right]$ \\
5 & $4-$ & 1258.9 & 1258.6 & 42 & $\left(n \mathrm{Bu}_{4} \mathrm{~N}\right)_{2} \mathrm{H}\left[\left(\alpha_{1}\right) \mathrm{Sn}\left(\mathrm{CH}_{2}\right)_{2} \mathrm{CO}_{2} \mathrm{R}\right]$ \\
6 & $4-$ & 1198.3 & 1198.3 & 100 & $\left(n \mathrm{Bu}_{4} \mathrm{~N}\right) \mathrm{H}_{2}\left[\left(\alpha_{1}\right) \mathrm{Sn}\left(\mathrm{CH}_{2}\right)_{2} \mathrm{CO}_{2} \mathrm{R}\right]$ \\
7 & $4-$ & 1138.0 & 1137.9 & 57 & $\mathrm{H}_{3}\left[\left(\alpha_{1}\right) \mathrm{Sn}\left(\mathrm{CH}_{2}\right)_{2} \mathrm{CO}_{2} \mathrm{R}\right]$ \\
8 & $5-$ & 958.4 & 958.4 & 73 & $\left(n \mathrm{Bu}_{4} \mathrm{~N}\right) \mathrm{H}\left[\left(\alpha_{1}\right) \mathrm{Sn}\left(\mathrm{CH}_{2}\right)_{2} \mathrm{CO}_{2} \mathrm{R}\right]$ \\
9 & $5-$ & 910.2 & 910.3 & 94 & $\mathrm{H}_{2}\left[\left(\alpha_{1}\right) \mathrm{Sn}\left(\mathrm{CH}_{2}\right)_{2} \mathrm{CO}_{2} \mathrm{R}\right]$ \\
\hline
\end{tabular}

$\mathrm{R}=\mathrm{CHPhCHPhNH}$

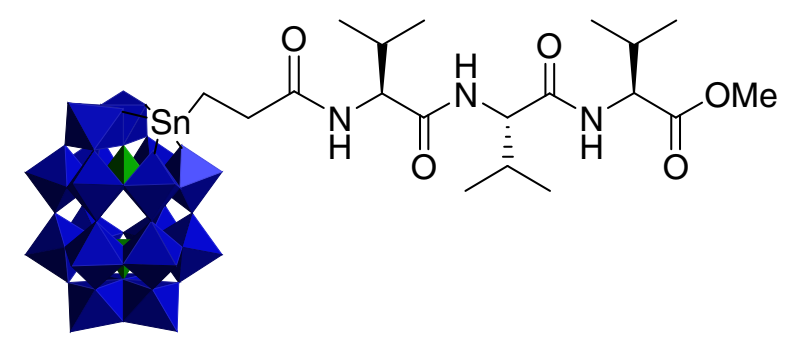

8d: $\quad \mathbf{T B A}_{7}\left[\alpha_{2}-\mathbf{P}_{2} \mathbf{W}_{17} \mathbf{O}_{61}\left\{\mathbf{S n C H}_{2} \mathbf{C H}_{2} \mathbf{C}(=\mathbf{O})\right.\right.$ Val-Val-Val-OMe $] . \quad$ To a solution of $\mathrm{TBA}_{6} \mathrm{H}\left[\alpha_{2}-\right.$ $\mathrm{P}_{2} \mathrm{~W}_{17} \mathrm{O}_{61}\left\{\mathrm{SnCH}_{2} \mathrm{CH}_{2} \mathrm{C}(=\mathrm{O})\right](\mathbf{4}, 0.034 \mathrm{mmol}, 200 \mathrm{mg})$, in acetonitrile $(3 \mathrm{~mL})$ was added the trifluoroacetic acid salt of the tri-valine $(0.068 \mathrm{mmol}, 30 \mathrm{mg}, 2$ equiv), triethylamine $(0.136 \mathrm{mmol}, 4$ equiv) and dimethylaminopyridine $(0.017 \mathrm{mmol}, 2 \mathrm{mg}, 0.5$ equiv.). The mixture was stirred at $\mathrm{rt}$ for 30 hours. A cationexchange resin (Amberlyst 15, 16-50 mesh, $\mathrm{TBA}^{+}$form) was added, followed by acetone $(10 \mathrm{~mL})$ and the mixture was stirred for 1 hour. The resin was filtered off and the filtrate was concentrated in vacuo. The oil which was obtained was dissolved in acetone $(2 \mathrm{~mL})$, and precipitated upon addition of $\mathrm{EtOH} / \mathrm{Et}_{2} \mathrm{O}$ $(2 \mathrm{~mL} / 30 \mathrm{~mL})$. The solid was isolated by centrifugation, washed with $\mathrm{Et}_{2} \mathrm{O}$ and dried in vacuo to afford the desired POM 8d as a white powder (183 mg, 84\%). IR: $\tilde{v}=2961(\mathrm{~m}), 2934(\mathrm{w}), 2872(\mathrm{~m}), 1648(\mathrm{w}), 1484$ (m), 1379 (w), 1088 (s), 1016 (w), 945 (s), 898 (s), 777 (vs) cm ${ }^{-1}$; ${ }^{1} \mathrm{H}$ NMR (400 MHz, CD ${ }_{3} \mathrm{CN}+10 \% \mathrm{D}_{2} \mathrm{O}$ ): $\delta 0.97\left(\mathrm{t}, J=7.2 \mathrm{~Hz}, 84 \mathrm{H}, \mathrm{N}\left[\left(\mathrm{CH}_{2}\right)_{3} \mathrm{Me}\right]_{4}\right), 1.08\left(\mathrm{~m}, 2 \mathrm{H}, \mathrm{SnCH}_{2}\right), 1.38-1.48\left(\mathrm{~m}, 56 \mathrm{H}, \mathrm{N}\left(\mathrm{CH}_{2} \mathrm{CH}_{2} \mathrm{CH}_{2} \mathrm{Me}\right)_{4}\right)$, 1.61-1.68 (m, $\left.56 \mathrm{H}, \mathrm{N}\left(\mathrm{CH}_{2} \mathrm{CH}_{2} \mathrm{CH}_{2} \mathrm{Me}\right)_{4}\right), 2.06\left(\mathrm{~m}, 3 \mathrm{H}, \mathrm{Me}_{2} \mathrm{CH}\right), 2.50$ (ddd, $J=2.2 \mathrm{~Hz}, J=6.7 \mathrm{~Hz}, J=11.1$ $\left.\mathrm{Hz}, 2 \mathrm{H}, \mathrm{CH}_{2} \mathrm{C}=\mathrm{O}\right), 3.14-3.20\left(\mathrm{~m}, 56 \mathrm{H}, \mathrm{N}\left(\mathrm{CH}_{2} \mathrm{Pr}\right)_{4}\right), 3.62(\mathrm{~s}, 3 \mathrm{H}, \mathrm{OMe}), 4.04(\mathrm{~m}, 1 \mathrm{H}, \mathrm{NHCH}), 4.14(\mathrm{~m}, 1 \mathrm{H}$, $\mathrm{NHCH}), 4.20(\mathrm{~m}, 1 \mathrm{H}, \mathrm{NHCH}), 7.37(\mathrm{~d}, J=8.6 \mathrm{~Hz}, 1 \mathrm{H}, \mathrm{NH}), 7.46(\mathrm{~d}, J=8.1 \mathrm{~Hz}, 1 \mathrm{H}, \mathrm{NH}), 7.59(\mathrm{~d}, J=8.1$ $\left.\mathrm{Hz}, 1 \mathrm{H}, \mathrm{NH}) ;{ }^{13} \mathrm{C} \mathrm{NMR}\left(50 \mathrm{MHz}, \mathrm{CD}_{3} \mathrm{CN}\right): \delta 14.0\left(\mathrm{~N}^{2} \mathrm{CH}_{2}\right]_{3} \mathrm{Me}\right), 18.5(\mathrm{MeCH}), 18.9(\mathrm{MeCH}), 19.2(\mathrm{MeCH})$,

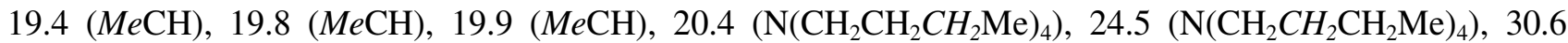
$\left(\mathrm{Me}_{2} \mathrm{CH}\right), 30.9\left(\mathrm{Me}_{2} \mathrm{CH}\right), 31.1\left(\mathrm{Me}_{2} \mathrm{CH}\right), 32.7\left(\mathrm{CH}_{2} \mathrm{C}=\mathrm{O}\right), 52.3(\mathrm{OMe}), 58.9(\mathrm{NHCH}), 59.2\left(\mathrm{~N}\left(\mathrm{CH}_{2} \mathrm{Pr}\right)_{4}\right), 59.5$ $(\mathrm{NHCH}), 61.0(\mathrm{NHCH}), 172.3(\mathrm{C}=\mathrm{O}), 172.7(\mathrm{C}=\mathrm{O}), 172.9(\mathrm{C}=\mathrm{O}), 176.7(\mathrm{C}=\mathrm{O}) ;{ }^{31} \mathrm{P}$ NMR $(162 \mathrm{MHz}$, $\left.\mathrm{CD}_{3} \mathrm{CN}\right): \delta-12.8(\mathrm{~s}, 1 \mathrm{P}),-9.5\left(\mathrm{~s}+\mathrm{d}, 1 \mathrm{P}, J_{S n P}=27.3 \mathrm{~Hz}\right) ;[\alpha]_{\mathrm{D}}{ }^{20}+1.1$ (c 1, Acetone); elemental analysis (\%) for $\mathrm{C}_{131} \mathrm{H}_{286} \mathrm{~N}_{10} \mathrm{O}_{66} \mathrm{P}_{2} \mathrm{SnW}_{17}\left(6363.8 \mathrm{~g} \cdot \mathrm{mol}^{-1}\right)$ : calc. C 24.72, H 4.53, N 2.20; found C 24.64, H 4.41, N 2.08. 


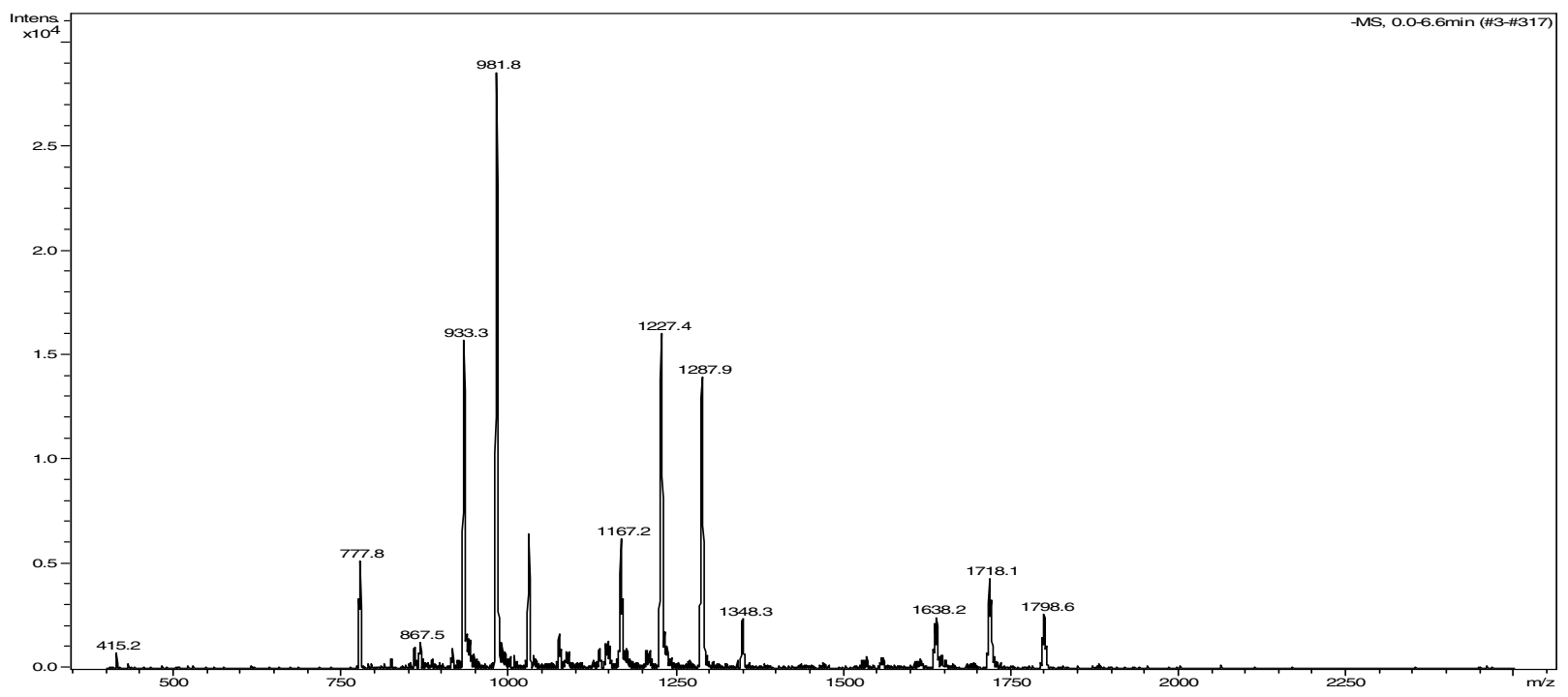

\begin{tabular}{cccccc}
\hline Entry & Charge & $\begin{array}{c}\text { Simulated } \\
\mathrm{m} / \mathrm{z}\end{array}$ & $\begin{array}{c}\text { Observed } \\
\mathrm{m} / \mathrm{z}\end{array}$ & $\begin{array}{c}\text { Relative } \\
\text { intensity }\end{array}$ & Composition \\
\hline 1 & $3-$ & 1798.3 & 1798.6 & 9.1 & $\left(n \mathrm{Bu}_{4} \mathrm{~N}\right)_{3} \mathrm{H}\left[\left(\alpha_{2}\right) \mathrm{Sn}\left(\mathrm{CH}_{2}\right)_{2} \mathrm{CO}_{2} \mathrm{R}\right]$ \\
2 & $3-$ & 1717.8 & 1718.1 & 14 & $\left(n \mathrm{Bu}_{4} \mathrm{~N}\right)_{2} \mathrm{H}_{2}\left[\left(\alpha_{2}\right) \mathrm{Sn}\left(\mathrm{CH}_{2}\right)_{2} \mathrm{CO}_{2} \mathrm{R}\right]$ \\
3 & $3-$ & 1637.4 & 1638.2 & 8.6 & $\left(n \mathrm{Bu}_{4} \mathrm{~N}\right) \mathrm{H}_{3}\left[\left(\alpha_{2}\right) \mathrm{Sn}\left(\mathrm{CH}_{2}\right)_{2} \mathrm{CO}_{2} \mathrm{R}\right]$ \\
4 & $4-$ & 1348.5 & 1348.3 & 7.4 & $\left(n \mathrm{Bu}_{4} \mathrm{~N}\right)_{3}\left[\left(\alpha_{2}\right) \mathrm{Sn}\left(\mathrm{CH}_{2}\right)_{2} \mathrm{CO}_{2} \mathrm{R}\right]$ \\
5 & $4-$ & 1288.1 & 1287.9 & 49 & $\left(n \mathrm{Bu}_{4} \mathrm{~N}\right)_{2} \mathrm{H}\left[\left(\alpha_{2}\right) \mathrm{Sn}\left(\mathrm{CH}_{2}\right)_{2} \mathrm{CO}_{2} \mathrm{R}\right]$ \\
6 & $4-$ & 1227.8 & 1227.4 & 56 & $\left(n \mathrm{Bu}_{4} \mathrm{~N}\right) \mathrm{H}_{2}\left[\left(\alpha_{2}\right) \mathrm{Sn}\left(\mathrm{CH}_{2}\right)_{2} \mathrm{CO}_{2} \mathrm{R}\right]$ \\
7 & $4-$ & 1167.2 & 1167.2 & 22 & $\mathrm{H}_{3}\left[\left(\alpha_{2}\right) \mathrm{Sn}\left(\mathrm{CH}_{2}\right)_{2} \mathrm{CO}_{2} \mathrm{R}\right]$ \\
8 & $5-$ & 1030.3 & 1030.7 & 23 & $\left(n \mathrm{Bu}_{4} \mathrm{~N}\right)_{2}\left[\left(\alpha_{2}\right) \mathrm{Sn}\left(\mathrm{CH}_{2}\right)_{2} \mathrm{CO}_{2} \mathrm{R}\right]$ \\
9 & $5-$ & 982.1 & 981.8 & 100 & $\left(n \mathrm{Bu}_{4} \mathrm{~N}\right) \mathrm{H}\left[\left(\alpha_{2}\right) \mathrm{Sn}\left(\mathrm{CH}_{2}\right)_{2} \mathrm{CO}_{2} \mathrm{R}\right]$ \\
10 & $5-$ & 933.6 & 933.3 & 55 & $\mathrm{H}_{2}\left[\left(\alpha_{2}\right) \mathrm{Sn}\left(\mathrm{CH}_{2}\right)_{2} \mathrm{CO}_{2} \mathrm{R}\right]$ \\
11 & $6-$ & 777.8 & 777.8 & 18 & $\mathrm{H}\left[\left(\alpha_{2}\right) \mathrm{Sn}\left(\mathrm{CH}_{2}\right)_{2} \mathrm{CO}_{2} \mathrm{R}\right]$ \\
\hline
\end{tabular}

$\mathrm{R}=\mathrm{Val}-\mathrm{Val}-\mathrm{Val}-\mathrm{OMe}$

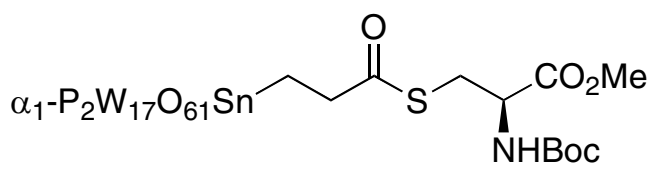

8f: $\mathrm{TBA}_{6.7} \mathrm{H}_{0.3}\left[\alpha_{1}-\mathbf{P}_{\mathbf{2}} \mathrm{W}_{\mathbf{1 7}} \mathrm{O}_{\mathbf{6 1}}\left[\mathrm{SnCH}_{\mathbf{2}} \mathrm{CH}_{\mathbf{2}} \mathrm{C}(=\mathbf{O}) \mathrm{SCH}_{\mathbf{2}} \mathrm{CH}(\mathrm{NHBoc}) \mathrm{CO}_{2} \mathrm{Me}\right]\right.$. Following GPB, 8f was isolated as a white powder $(200 \mathrm{mg}, 73 \%)$. IR: $\tilde{v}=2960(\mathrm{~m}), 2935(\mathrm{w}), 2872(\mathrm{~m}), 1708(\mathrm{w}), 1638(\mathrm{w}), 1483(\mathrm{~m})$, 1379 (w), 1158 (w), 1085 (s), 1010 (w), 946 (s), 906 (s), 780 (vs) cm ${ }^{-1} ;{ }^{1} \mathrm{H}$ NMR (400 MHz, CD ${ }_{3} \mathrm{CN}$ ): $\delta 0.96$ (t, $\left.J=7.2 \mathrm{~Hz}, 84 \mathrm{H}, \mathrm{N}\left[\left(\mathrm{CH}_{2}\right)_{3} \mathrm{Me}\right]_{4}\right), 1.26\left(\mathrm{~m}, 2 \mathrm{H}, \mathrm{SnCH}_{2}\right), 1.38-1.48\left(\mathrm{~m}, 67 \mathrm{H}, \mathrm{N}\left(\mathrm{CH}_{2} \mathrm{CH}_{2} \mathrm{CH}_{2} \mathrm{Me}\right)_{4}+\mathrm{O} t \mathrm{Bu}\right)$, 1.61-1.68 (m, $\left.56 \mathrm{H}, \mathrm{N}\left(\mathrm{CH}_{2} \mathrm{CH}_{2} \mathrm{CH}_{2} \mathrm{Me}\right)_{4}\right), 2.91$ (m, $\left.2 \mathrm{H}, \mathrm{CH}_{2} \mathrm{C}=\mathrm{O}\right), 3.14-3.20$ (m, $\left.56 \mathrm{H}, \mathrm{N}\left(\mathrm{CH}_{2} \mathrm{Pr}\right)_{4}\right), 3.31$ (dd, $J=4.6 \mathrm{~Hz}, J=13.9 \mathrm{~Hz}, 1 \mathrm{H}, \mathrm{SCH}), 3.66(\mathrm{~s}, 3 \mathrm{H}, \mathrm{OMe}), 4.24(\mathrm{~m}, 1 \mathrm{H}, \mathrm{CH}), 5.83(\mathrm{~d}, J=7.8 \mathrm{~Hz}, 1 \mathrm{H}, \mathrm{NH}) ;{ }^{13} \mathrm{C}$

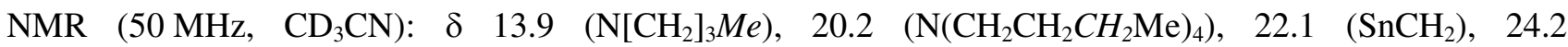
$\left(\mathrm{N}\left(\mathrm{CH}_{2} \mathrm{CH}_{2} \mathrm{CH}_{2} \mathrm{Me}\right)_{4}\right), 28.3\left(\mathrm{OCMe}_{3}\right), 30.7 \quad\left(\mathrm{CH}_{2} \mathrm{C}=\mathrm{O}\right), 41.1 \quad\left(\mathrm{SCH}_{2}\right), 52.8(\mathrm{OMe}), 54.2(\mathrm{CH}), 58.9$ $\left(\mathrm{N}\left(\mathrm{CH}_{2} \mathrm{Pr}\right)_{4}\right), 80.0\left(\mathrm{OCMe}_{3}\right), 156.1\left(\mathrm{NHCO}_{2}\right), 171.9\left(\mathrm{CO}_{2} \mathrm{Me}\right), 202.6\left(\mathrm{CH}_{2} \mathrm{C}=\mathrm{O}\right) ;{ }^{31} \mathrm{P} \mathrm{NMR}(162 \mathrm{MHz}$, $\left.\mathrm{CD}_{3} \mathrm{CN}\right): \delta-12.1(\mathrm{~s}, 1 \mathrm{P}),-6.7\left(\mathrm{~s}+\mathrm{d}, 1 \mathrm{P}, J_{S n P}=43 \mathrm{~Hz}\right)$; elemental analysis $(\%)$ for $\mathrm{C}_{119.2} \mathrm{H}_{261.5} \mathrm{~N}_{7.7} \mathrm{O}_{66} \mathrm{P}_{2} \mathrm{SnW}_{17}$ $\left(6197.3 \mathrm{~g} \cdot \mathrm{mol}^{-1}\right)$ : calc. C $23.10, \mathrm{H} 4.25, \mathrm{~N} 1.74$; found C 23.21, H 4.24, N 1.68 . 
<smiles></smiles>

8e: $\mathrm{TBA}_{6.8} \mathrm{H}_{0.2}\left[\alpha_{2}-\mathrm{P}_{2} \mathrm{~W}_{17} \mathrm{O}_{61}\left\{\mathrm{SnCH}_{2} \mathrm{CH}_{2} \mathrm{C}(=\mathbf{O}) \mathrm{SCH}_{2} \mathrm{CH}(\mathrm{NHBoc}) \mathrm{CO}_{2} \mathrm{Me}\right]\right.$. Following GPB, 8e was isolated as a white powder $(228 \mathrm{mg}, 84 \%)$. IR: $\tilde{v}=2960(\mathrm{~m}), 2934(\mathrm{w}), 2872(\mathrm{~m}), 1709(\mathrm{w}), 1483(\mathrm{~m}), 1379(\mathrm{w})$, 1166 (w), 1088 (s), 1016 (w), 944 (s), 900 (s), 778 (vs) cm ${ }^{-1}$; ${ }^{1} \mathrm{H}$ NMR (400 MHz, CD ${ }_{3} \mathrm{CN}$ ): $\delta 0.97$ (t, $\left.J=7.2 \mathrm{~Hz}, 84 \mathrm{H}, \mathrm{N}\left[\left(\mathrm{CH}_{2}\right)_{3} \mathrm{Me}\right]_{4}\right), 1.38-1.48\left(\mathrm{~m}, 67 \mathrm{H}, \mathrm{N}\left(\mathrm{CH}_{2} \mathrm{CH}_{2} \mathrm{CH}_{2} \mathrm{Me}\right)_{4}+\mathrm{O} t B u+\mathrm{SnCH}_{2}\right), 1.61-1.68(\mathrm{~m}$, $\left.56 \mathrm{H}, \mathrm{N}\left(\mathrm{CH}_{2} \mathrm{CH}_{2} \mathrm{CH}_{2} \mathrm{Me}\right)_{4}\right), 2.77\left(\mathrm{t}, J=8.6 \mathrm{~Hz}, 2 \mathrm{H}, \mathrm{CH}_{2} \mathrm{C}=\mathrm{O}\right), 3.14-3.20\left(\mathrm{~m}, 56 \mathrm{H}, \mathrm{N}\left(\mathrm{CH}_{2} \mathrm{Pr}\right)_{4}\right), 3.35(\mathrm{dd}, J=$ $4.8 \mathrm{~Hz}, J=13.9 \mathrm{~Hz}, 1 \mathrm{H}, \mathrm{SCH}), 3.66(\mathrm{~s}, 3 \mathrm{H}, \mathrm{OMe}), 4.25(\mathrm{~m}, 1 \mathrm{H}, \mathrm{CH}), 5.74(\mathrm{~d}, J=8.1 \mathrm{~Hz}, 1 \mathrm{H}, \mathrm{NH}) ;{ }^{13} \mathrm{C}$

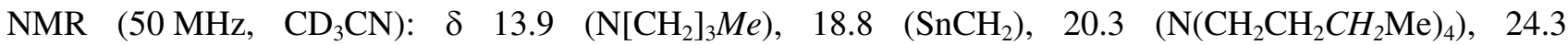
$\left(\mathrm{N}\left(\mathrm{CH}_{2} \mathrm{CH}_{2} \mathrm{CH}_{2} \mathrm{Me}\right)_{4}\right), 28.4\left(\mathrm{OCMe}_{3}\right), 30.8\left(\mathrm{CH}_{2} \mathrm{C}=\mathrm{O}\right), 40.3\left(\mathrm{SCH}_{2}\right), 52.9(\mathrm{OMe}), 54.2(\mathrm{CH}), 59.0$ $\left(\mathrm{N}\left(\mathrm{CH}_{2} \mathrm{Pr}\right)_{4}\right), 80.0\left(\mathrm{OCMe}_{3}\right), 156.1\left(\mathrm{NHCO}_{2}\right), 171.9\left(\mathrm{CO}_{2} \mathrm{Me}\right), 201.5\left(\mathrm{CH}_{2} \mathrm{C}=\mathrm{O}\right) ;{ }^{31} \mathrm{P}$ NMR $(162 \mathrm{MHz}$, $\left.\mathrm{CD}_{3} \mathrm{CN}\right): \delta-12.4(\mathrm{~s}, 1 \mathrm{P}),-9.4\left(\mathrm{~s}+\mathrm{d}, 1 \mathrm{P}, J_{S n P}=25 \mathrm{~Hz}\right) ;[\alpha]_{\mathrm{D}}{ }^{20}-1.0$ (c 1, Acetone); elemental analysis (\%) for $\mathrm{C}_{120.8} \mathrm{H}_{265} \mathrm{~N}_{7.8} \mathrm{O}_{66} \mathrm{P}_{2} \mathrm{SnW}_{17}\left(6221.4 \mathrm{~g} \cdot \mathrm{mol}^{-1}\right)$ : calc. C 23.32, H 4.29, N 1.76; found C 23.33, H 4.12, N 1.75.

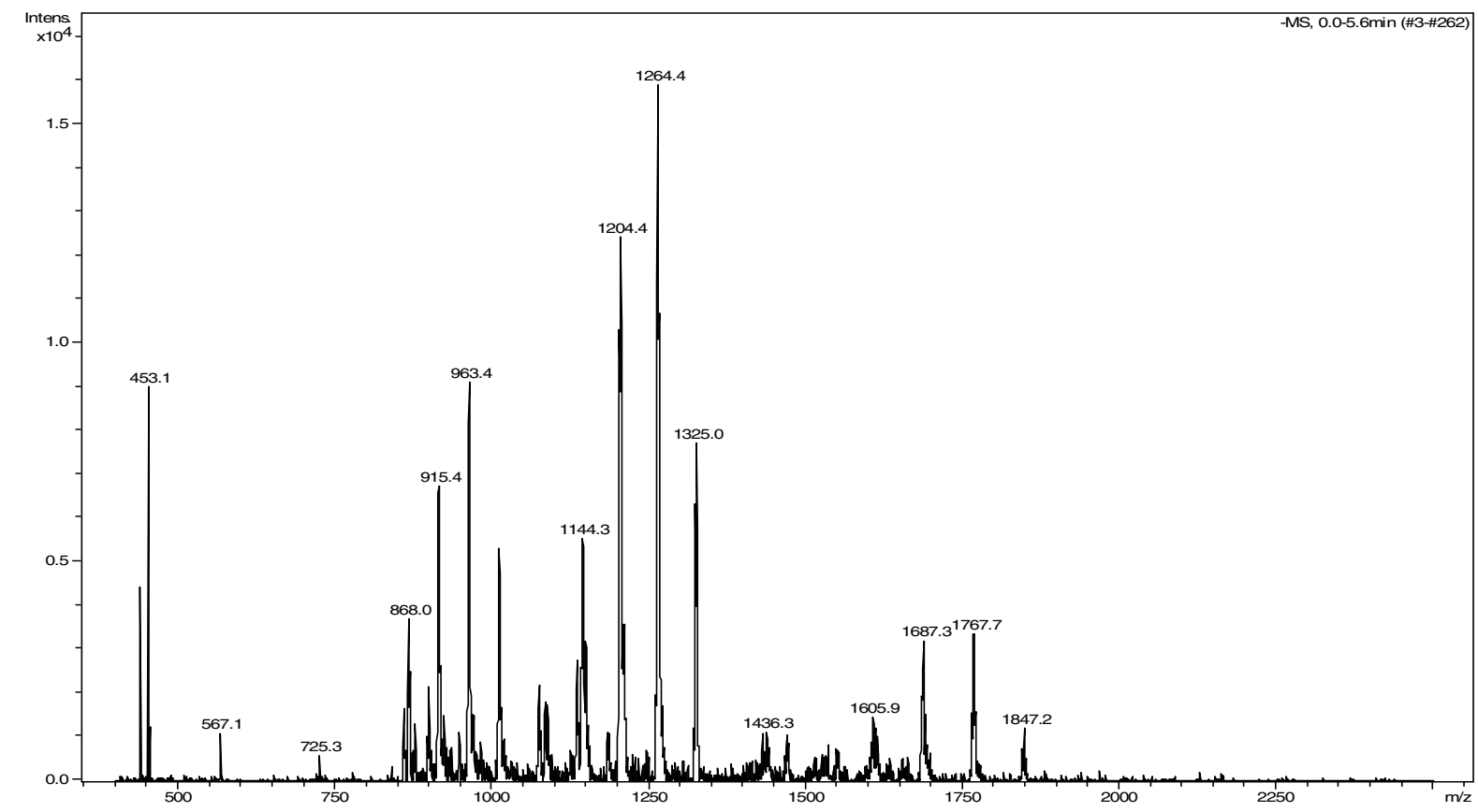

\begin{tabular}{cccccc}
\hline Entry & Charge & $\begin{array}{c}\text { Simulated } \\
\mathrm{m} / \mathrm{z}\end{array}$ & $\begin{array}{c}\text { Observed } \\
\mathrm{m} / \mathrm{z}\end{array}$ & $\begin{array}{c}\text { Relative } \\
\text { intensity }\end{array}$ & Composition \\
\hline 1 & $3-$ & 1847.3 & 1847.3 & 7.1 & $\left(n \mathrm{Bu}_{4} \mathrm{~N}\right)_{4}\left[\left(\alpha_{2}\right) \mathrm{Sn}\left(\mathrm{CH}_{2}\right)_{2} \mathrm{CO}_{2} \mathrm{R}\right]$ \\
2 & $3-$ & 1766.9 & 1767.7 & 21 & $\left(n \mathrm{Bu}_{4} \mathrm{~N}\right)_{3} \mathrm{H}\left[\left(\alpha_{2}\right) \mathrm{Sn}\left(\mathrm{CH}_{2}\right)_{2} \mathrm{CO}_{2} \mathrm{R}\right]$ \\
3 & $3-$ & 1686.5 & 1687.3 & 20 & $\left(n \mathrm{Bu}_{4} \mathrm{~N}\right)_{2} \mathrm{H}_{2}\left[\left(\alpha_{2}\right) \mathrm{Sn}\left(\mathrm{CH}_{2}\right)_{2} \mathrm{CO}_{2} \mathrm{R}\right]$ \\
4 & $3-$ & 1606.0 & 1605.9 & 9.3 & $\left(n \mathrm{Bu}_{4} \mathrm{~N}\right) \mathrm{H}_{3}\left[\left(\alpha_{2}\right) \mathrm{Sn}\left(\mathrm{CH}_{2}\right)_{2} \mathrm{CO}_{2} \mathrm{R}\right]$ \\
5 & $4-$ & 1324.9 & 1325.0 & 49 & $\left(n \mathrm{Bu}_{4} \mathrm{~N}\right)_{3}\left[\left(\alpha_{2}\right) \mathrm{Sn}\left(\mathrm{CH}_{2}\right)_{2} \mathrm{CO}_{2} \mathrm{R}\right]$ \\
6 & $4-$ & 1264.6 & 1264.4 & 100 & $\left(n \mathrm{Bu}_{4} \mathrm{~N}\right)_{2} \mathrm{H}\left[\left(\alpha_{2}\right) \mathrm{Sn}\left(\mathrm{CH}_{2}\right)_{2} \mathrm{CO}_{2} \mathrm{R}\right]$ \\
7 & $4-$ & 1204.3 & 1204.4 & 78 & $\left(n \mathrm{Bu}_{4} \mathrm{~N}\right) \mathrm{H}_{2}\left[\left(\alpha_{2}\right) \mathrm{Sn}\left(\mathrm{CH}_{2}\right)_{2} \mathrm{CO}_{2} \mathrm{R}\right]$ \\
8 & $4-$ & 1143.7 & 1144.3 & 35 & $\mathrm{H}_{3}\left[\left(\alpha_{2}\right) \mathrm{Sn}\left(\mathrm{CH}_{2}\right)_{2} \mathrm{CO}_{2} \mathrm{R}\right]$ \\
9 & $5-$ & 1011.5 & 1011.5 & 33 & $\left(n \mathrm{Bu}_{4} \mathrm{~N}\right)_{2}\left[\left(\alpha_{2}\right) \mathrm{Sn}\left(\mathrm{CH}_{2}\right)_{2} \mathrm{CO}_{2} \mathrm{R}\right]$ \\
10 & $5-$ & 963.2 & 963.4 & 56 & $\left(n \mathrm{Bu}_{4} \mathrm{~N}\right) \mathrm{H}\left[\left(\alpha_{2}\right) \mathrm{Sn}\left(\mathrm{CH}_{2}\right)_{2} \mathrm{CO}_{2} \mathrm{R}\right]$ \\
11 & $5-$ & 914.8 & 915.4 & 42 & $\mathrm{H}_{2}\left[\left(\alpha_{2}\right) \mathrm{Sn}\left(\mathrm{CH}_{2}\right)_{2} \mathrm{CO}_{2} \mathrm{R}\right]$ \\
\hline
\end{tabular}




\section{Cartesian Data for all the calculated polyoxometalates}

\subsection{Models of Sn-Substituted $\alpha_{2-}$ Dawson Heteropolyanion $\underline{4}$}

\subsubsection{Conf A}

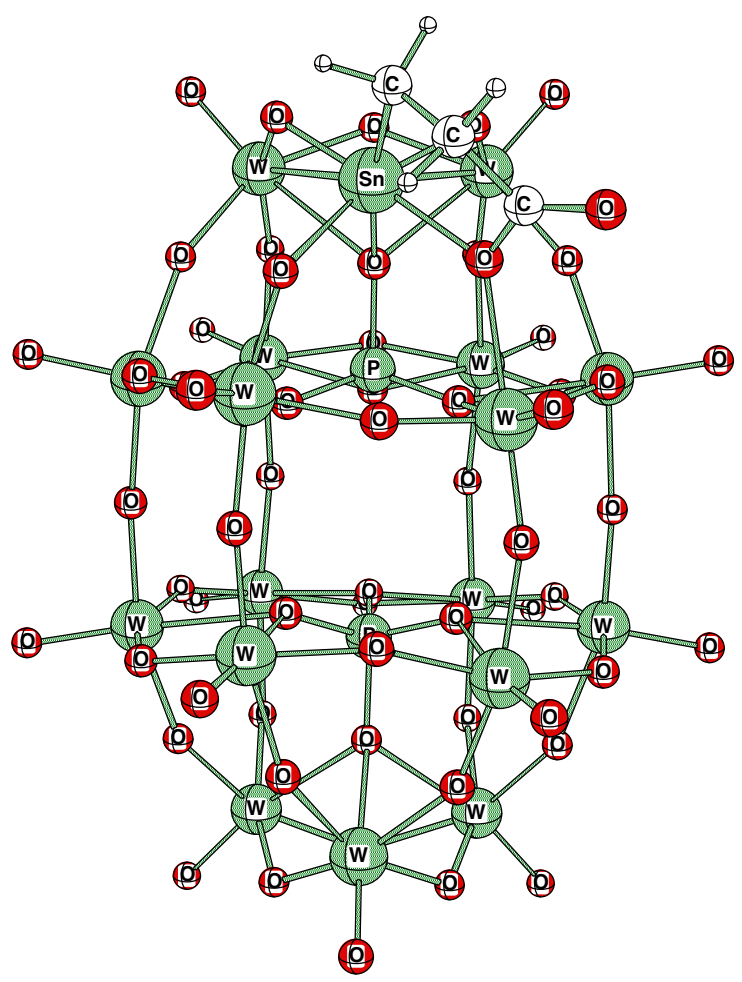

89

\begin{tabular}{lrrr}
\multicolumn{4}{l}{ Energy $=-6609.8828847760$} \\
W & -1.732681 & 2.235306 & -3.466873 \\
W & 1.757721 & 2.127335 & -3.465714 \\
W & 1.648605 & -1.688110 & -3.986160 \\
W & 3.629395 & 1.809418 & -0.238190 \\
W & 1.977005 & 1.119461 & 2.751328 \\
W & -1.820129 & -1.570847 & -3.955688 \\
W & -1.845947 & 1.493607 & 2.788177 \\
W & 1.899582 & -2.679672 & 2.202225 \\
W & -1.843887 & -2.349152 & 2.280991 \\
W & -3.578568 & 1.818542 & -0.213272 \\
W & -3.627701 & -2.005371 & -0.677139 \\
W & 1.668259 & -5.006302 & -2.172272 \\
$W$ & 1.783508 & 5.062134 & -1.018036 \\
$W$ & 3.580673 & -2.020401 & -0.761749 \\
W & -1.754715 & 5.096527 & -0.967896 \\
W & -0.184723 & -5.449493 & 0.751312 \\
W & -1.834656 & -4.954880 & -2.255890 \\
$O$ & 1.678741 & 3.850006 & -2.551056 \\
$O$ & -2.008286 & -0.471710 & 2.518677 \\
$O$ & 3.018219 & 3.624390 & -0.355057 \\
$O$ & 0.104019 & 1.108765 & 3.025070 \\
$O$ & -3.024475 & -1.472839 & -2.446259 \\
$O$ & 0.029175 & 1.684540 & -1.778778
\end{tabular}

$\begin{array}{llll}\mathrm{O} & 1.570221 & 0.247391 & -3.917755\end{array}$

$\begin{array}{llll}O & -2.959412 & 1.734238 & -2.043839\end{array}$

$\begin{array}{llll}O & -3.467209 & -2.465744 & 1.212952\end{array}$

$\begin{array}{llll}0 & -1.637268 & 0.331680 & -3.909973\end{array}$

$\begin{array}{llll}O & -1.309158 & -1.782684 & 0.002594\end{array}$

$\begin{array}{llll}O & -1.763443 & -3.507858 & -3.503342\end{array}$

$\begin{array}{llll}\mathrm{O} & 3.516052 & 1.701660 & 1.707764\end{array}$

$\begin{array}{llll}0 & -0.126511 & -5.633316 & -2.841721\end{array}$

$\begin{array}{llll}O & 0.012039 & 2.490555 & -4.269775\end{array}$

O $3.694733 \quad-0.195251 \quad-0.269119$

$\begin{array}{llll}\mathrm{O} & -3.701752 & -0.107239 & -0.226746\end{array}$

$\begin{array}{llll}0 & -3.452332 & 1.795486 & 1.753464\end{array}$

$\begin{array}{llll}\text { O } & -0.095383 & -1.765656 & -4.842529\end{array}$

$\begin{array}{llll}\text { O } & -0.040970 & -1.530746 & -2.259842\end{array}$

$\begin{array}{llll}O & -1.290894 & 1.429886 & 0.448578\end{array}$

\begin{tabular}{llll}
\hline & 3.486160 & -2.636353 & 1.105057
\end{tabular}

$\begin{array}{llll}\text { O } & 1.279282 & -4.345291 & 1.575958\end{array}$

$\begin{array}{llll}\text { O } & 2.920624 & -1.579422 & -2.510391\end{array}$

$\begin{array}{llll}\text { O } & 1.294266 & -1.865814 & -0.028152\end{array}$

$\begin{array}{llll}0 & -1.507950 & -5.944824 & -0.544754\end{array}$

$\begin{array}{llll}O & -2.925034 & 3.691452 & -0.303177\end{array}$

$\begin{array}{llll}\mathrm{O} & -3.007797 & -3.777756 & -1.213760\end{array}$

$\begin{array}{llll}\text { O } & 1.200867 & -5.986786 & -0.537582\end{array}$

$\begin{array}{lllll}\text { O } & 1.545044 & -3.583847 & -3.498993\end{array}$

$\begin{array}{lllll}\mathrm{O} & 0.035767 & 3.574432 & -0.015366\end{array}$

$\begin{array}{llll}O & 2.890701 & -3.876663 & -1.262207\end{array}$

$\begin{array}{llll}P & 0.020325 & 1.939484 & -0.240082\end{array}$

$\begin{array}{llll}\text { P } & -0.030045 & -2.171265 & -0.824677\end{array}$

$\begin{array}{llll}\text { O } & 1.554932 & 5.600113 & 0.788498\end{array}$

$\begin{array}{llll}O & 1.323639 & 1.434616 & 0.487305\end{array}$

$\begin{array}{llll}O & -2.786484 & 2.741028 & -4.768372\end{array}$

$\begin{array}{llll}\text { O } & 2.687057 & -1.868408 & -5.384064\end{array}$

$\begin{array}{llll}\text { O } & -0.077774 & -3.782089 & -1.032181\end{array}$

$\begin{array}{llll}\mathrm{O} & 2.985931 & 1.622986 & -2.058731\end{array}$

$\begin{array}{llll}O & 1.649094 & 3.449936 & 2.955063\end{array}$

$\begin{array}{llll}O & -1.324188 & 3.302139 & 2.683701\end{array}$

$\begin{array}{llll}O & -2.901001 & -1.716095 & -5.324432\end{array}$

$\begin{array}{llll}\text { O } & 2.209702 & -0.674042 & 2.470032\end{array}$

$\begin{array}{llll}\text { O } & -1.613724 & 3.924225 & -2.539856\end{array}$

$\begin{array}{llll}0 & -2.836105 & 6.348999 & -1.551804\end{array}$

$\begin{array}{llll}\text { O } & -1.326706 & -4.183838 & 1.605133\end{array}$

$\begin{array}{llll}O & -2.954926 & -6.070435 & -3.019272\end{array}$

$\begin{array}{llll}O & 0.035385 & 5.778839 & -1.490570\end{array}$

$\begin{array}{llll}O & 0.067154 & -2.237930 & 2.625778\end{array}$

O $\quad 5.269348 \quad-2.354385-1.079910$

$\begin{array}{llll}\text { O } & 2.581940 & 1.239822 & 4.387192\end{array}$

$\begin{array}{llll}\text { O } & -2.489914 & 1.511993 & 4.419189\end{array}$

$\begin{array}{llll}\mathrm{O} & -5.278671 & 2.191757 & -0.388382\end{array}$

$\begin{array}{llll}\text { O } & 2.869370 & 6.311478 & -1.601669\end{array}$

$\begin{array}{llll}\mathrm{O} & 2.822861 & 2.606461 & -4.768372\end{array}$

$\begin{array}{llll}O & -1.439423 & 5.721298 & 0.805649\end{array}$

$\begin{array}{llll}O & -5.341980 & -2.269882 & -0.910461\end{array}$

Sn $\quad 0.007416 \quad 4.699753 \quad 1.911835$

$\begin{array}{lllll}\text { O } & 2.711670 & -6.185287 & -2.950684\end{array}$

O $5.341965 \quad 2.095520 \quad-0.442123$

$\begin{array}{llll}\text { O } & -2.469360 & -2.901321 & 3.820129\end{array}$

$\begin{array}{llll}\text { O } & -0.224060 & -6.839005 & 1.824493\end{array}$ 


$\begin{array}{rrrr}\text { O } & 2.570953 & -3.185974 & 3.737595 \\ \mathrm{C} & 0.299759 & 5.923187 & 3.698578 \\ \mathrm{C} & 1.162338 & 5.093079 & 4.655960 \\ \mathrm{C} & 2.184479 & 4.163086 & 3.954193 \\ \mathrm{O} & 3.340363 & 4.089050 & 4.345825 \\ \mathrm{H} & -0.687897 & 6.200287 & 4.125595 \\ \mathrm{H} & 0.822891 & 6.838989 & 3.347351 \\ \mathrm{H} & 1.739578 & 5.711517 & 5.384064 \\ \mathrm{H} & 0.505096 & 4.408432 & 5.241730\end{array}$

\subsubsection{Conf B}

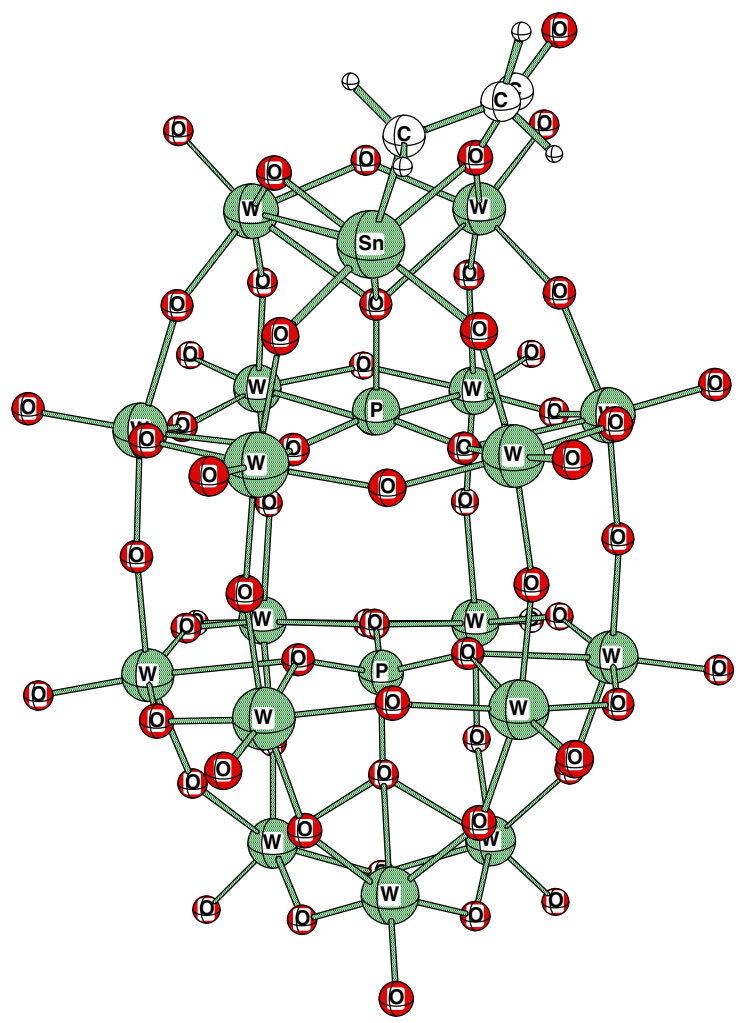

89

Energy $=-6609.8918413450$

$\begin{array}{cccc}\text { O } & 1.464798 & 5.362793 & 1.677795 \\ \text { Sn } & -0.088028 & 3.984863 & 2.738419 \\ \text { C } & 0.390976 & 5.435608 & 4.306412 \\ \text { C } & 1.786880 & 5.973465 & 3.975372 \\ \text { C } & 2.026367 & 6.265244 & 2.473206 \\ \text { O } & 1.479156 & 2.694107 & 3.228119 \\ \text { W } & -1.927658 & 2.054947 & -2.785019 \\ \text { W } & 1.547089 & 2.010849 & -3.079437 \\ \text { W } & 1.598145 & -1.750946 & -3.841782 \\ \text { W } & 3.534836 & 1.550507 & 0.145493 \\ \text { W } & 1.993652 & 0.877319 & 3.208878 \\ \text { W } & -1.864120 & -1.705643 & -3.607880 \\ \text { W } & -1.767288 & 0.741257 & 3.425919 \\ \text { W } & 2.100998 & -2.870560 & 2.313171 \\ \text { W } & -1.669495 & -2.992020 & 2.531052 \\ \text { W } & -3.622360 & 1.296387 & 0.501007 \\ \text { W } & -3.555542 & -2.465546 & -0.329895 \\ \text { W } & 1.812363 & -5.174515 & -2.260910 \\ \text { W } & 1.586884 & 4.750610 & -0.490524 \\ \text { W } & 3.644165 & -2.204636 & -0.724930\end{array}$

\begin{tabular}{|c|c|c|c|}
\hline & 1 & & \\
\hline & 0.195419 & -5.761246 & 0.760788 \\
\hline & -1.668533 & -5.208603 & \\
\hline & 1.451538 & 3.830963 & -2.07020 \\
\hline & -1.950806 & -1.147217 & 2.89465 \\
\hline & 2.788773 & 3.420364 & 0.21485 \\
\hline & 0.142593 & 0.404388 & 3.583557 \\
\hline & -3.031143 & -1.776169 & $-2.0688 \mathrm{~s}$ \\
\hline & -0.089874 & 1.502808 & $-1.28617 \mathrm{c}$ \\
\hline & 1.431107 & 0.234085 & -3.639359 \\
\hline & -3.054642 & 1.399658 & -1.3692 \\
\hline & -3.320114 & -3.087234 & 1.49275 \\
\hline & -1.752975 & 0.198929 & -3.406296 \\
\hline & -1.219055 & -2.199356 & 0.2557 \\
\hline & -1.696594 & -3.665634 & -3.30423 \\
\hline & 3.542847 & 1.356949 & 2.05522 \\
\hline & 0.061844 & -5.763046 & -2.909439 \\
\hline & -0.236237 & 2.451599 & -3.693375 \\
\hline & 3.719086 & -0.351517 & -0.130341 \\
\hline & -3.684341 & -0.620773 & 0.281998 \\
\hline & -3.417282 & 1.115128 & 2.414749 \\
\hline & -0.175934 & -1.760986 & -4.609375 \\
\hline & -0.039429 & -1.714233 & -2.031174 \\
\hline & -1.278824 & 1.010605 & 0.998901 \\
\hline & 3.641846 & 1064 & 9131 \\
\hline & 1.534302 & -4.617310 & 1.536865 \\
\hline & 2.899216 & -1.599884 & -2.407211 \\
\hline & 1.385925 & -2.103683 & 0.116180 \\
\hline & -1.161255 & -6.270935 & -0.568710 \\
\hline & -3.050415 & 3.208328 & 0.508911 \\
\hline & -2.879166 & -4.178177 & 4307 \\
\hline & 1.525803 & -6.214050 & -0.674683 \\
\hline & 1.609131 & -3.627060 & -3.498413 \\
\hline & -0.017365 & 3.221069 & 0.643265 \\
\hline & 3.03 & -3.984756 & 2697 \\
\hline & -0.020737 & 1.591675 & 0.274307 \\
\hline & 0.049698 & -2.444901 & -0.639420 \\
\hline & 1.318954 & 3091 & 0.852890 \\
\hline & -3.070450 & 479 & -3.9 \\
\hline & 2.579758 & -1.738251 & -5.291870 \\
\hline & 0.089874 & -4.036926 & -0.959229 \\
\hline & 2.799927 & 1.621490 & -1.683167 \\
\hline & -1.403275 & 2.607788 & 1776 \\
\hline & -2.992325 & -1.781326 & -4.943924 \\
\hline & 2.279709 & -1.009750 & 2.686554 \\
\hline & -1.806152 & 3.6 & -1.741104 \\
\hline & -3.082779 & 5.963257 & -0.465134 \\
\hline & -1.094757 & -4.714920 & 1.695938 \\
\hline & -2.744217 & -6.335999 & -2.952164 \\
\hline & -0.184982 & 5.511658 & -0.560669 \\
\hline & 0.227570 & -2.757889 & 2.816605 \\
\hline & 5.338257 & -2.416824 & -1.109924 \\
\hline & 2.770157 & 0.798737 & 4.777939 \\
\hline & -2.380219 & 0.558548 & 5.055710 \\
\hline & -5.338272 & 1.627029 & 0.414490 \\
\hline & 2.600922 & 6.104767 & -0.938080 \\
\hline & 2.540985 & 2.666107 & -4.36285 \\
\hline & -1.549667 & 5.129944 & 1.753296 \\
\hline & -5.263199 & -2.788818 & -0.537369 \\
\hline & 2.884064 & -6.228180 & \\
\hline
\end{tabular}




$\begin{array}{rrrr}\mathrm{O} & 5.202530 & 2.018005 & -0.102600 \\ \mathrm{O} & -2.219589 & -3.677795 & 4.044174 \\ \mathrm{O} & 0.285889 & -7.225021 & 1.725571 \\ \mathrm{O} & 2.856308 & -3.522552 & 3.751068 \\ \mathrm{O} & 2.700760 & 7.225021 & 2.112778 \\ \mathrm{H} & 0.351761 & 4.924271 & 5.291855 \\ \mathrm{H} & -0.388763 & 6.227722 & 4.263245 \\ \mathrm{H} & 2.050674 & 6.909225 & 4.524704 \\ \mathrm{H} & 2.543427 & 5.201080 & 4.250458\end{array}$

\subsection{Models of Sn-Substituted $\alpha_{1^{-}}$ Dawson Heteropolyanion 1}

\subsection{1 $\underline{\text { Conf } 1}$}

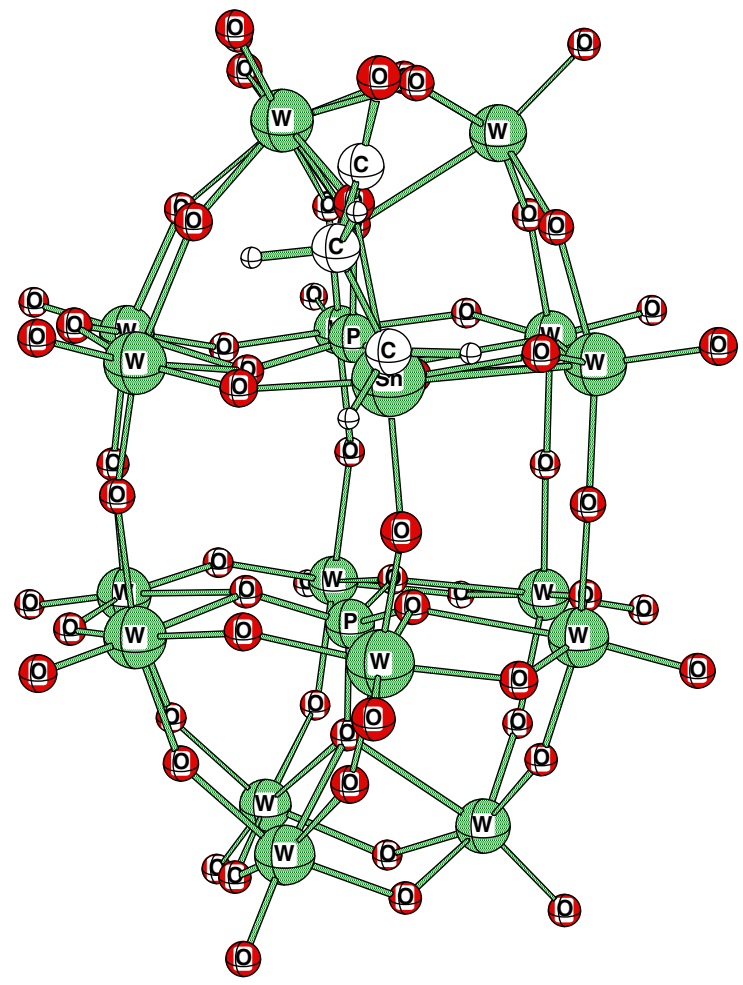

89

\begin{tabular}{lrrr}
\multicolumn{4}{l}{ Energy $=-6609.8904982780$} \\
W & -3.246948 & 1.726196 & 1.272873 \\
W & -3.357468 & 2.156525 & -2.195221 \\
W & -3.386841 & -1.676743 & -2.584152 \\
W & -0.168594 & 2.232132 & -4.252502 \\
W & 2.881546 & 2.134537 & -2.538300 \\
W & -3.250595 & -2.098083 & 0.865265 \\
W & 3.133820 & 1.732437 & 1.223618 \\
W & 2.849792 & -1.688751 & -2.975922 \\
W & 2.982727 & -2.104279 & 0.774353 \\
Sn & 0.150436 & 1.511246 & 2.923553 \\
W & 0.068054 & -2.333420 & 2.663956 \\
W & -1.280594 & -4.825455 & -3.000992 \\
W & -1.107208 & 5.212265 & -2.133270 \\
W & -0.216370 & -1.580383 & -4.605820 \\
W & -1.238586 & 5.080750 & 1.322845 \\
W & 1.821701 & -4.982056 & -1.410217
\end{tabular}

\begin{tabular}{|c|c|c|c|}
\hline & 031 & 85 & 23 \\
\hline & -2.586136 & 3.962692 & -2.010208 \\
\hline & 3.048706 & -0.208984 & \\
\hline & -0.368530 & 4.058228 & -3.42482 \\
\hline & 3.287262 & 1.642563 & -0.7254 \\
\hline & -1.770996 & -1.948975 & 2.081741 \\
\hline & -1.643188 & 1.612564 & -0.506439 \\
\hline & -3.564484 & 0.232773 & -2.26030 \\
\hline & -1.858246 & 1.388550 & 2.475723 \\
\hline & 1.985382 & -2.579422 & 2.370239 \\
\hline & -3.503769 & -0.129974 & 0.944992 \\
\hline & 0.655807 & -1.708038 & 0.322083 \\
\hline & -2.597260 & -3.899857 & 0.521500 \\
\hline & 1.744385 & 2.491547 & -4.085052 \\
\hline & -1.778290 & -5.715195 & -1.353348 \\
\hline & -4.112869 & 2.250137 & -0.429367 \\
\hline & 0.035934 & 0.373322 & -4.542343 \\
\hline & 0.331635 & -0.535950 & 3.051956 \\
\hline & 2.220398 & 1.877533 & 2.863037 \\
\hline & -4.171997 & -2.094315 & -0.862869 \\
\hline & -1.654678 & -1.556793 & -0.878174 \\
\hline & 0.629181 & 1.573883 & 0.782135 \\
\hline & 1.719757 & -1.773712 & -4.564560 \\
\hline & 2.427979 & -3.592331 & -2.601440 \\
\hline & -1.970184 & -1.268463 & -3.852448 \\
\hline & 0.546600 & -1.444565 & -2.282578 \\
\hline & 0.613113 & -5.87 & -0.118408 \\
\hline & -0.336365 & 2395 & 3.021545 \\
\hline & -0.284149 & -4.072571 & 1.741180 \\
\hline & 0.511139 & -5.562027 & -2.776245 \\
\hline & -2.685791 & -3.514343 & -2.735184 \\
\hline & -0.146347 & 705 & -0.393036 \\
\hline & -0.489212 & -3.429276 & -4.134003 \\
\hline & -0.154160 & 2.092300 & -0.523102 \\
\hline & -0.153061 & -2.0 & 3317 \\
\hline & 0.624893 & 5.8 & -1.588882 \\
\hline & 0.595093 & 1.720474 & -1.840347 \\
\hline & -4.596878 & 2.054779 & 2.341949 \\
\hline & -4.786514 & 5950 & 1450 \\
\hline & -0.178528 & -3.632507 & -1.169525 \\
\hline & -1.921814 & 1.952866 & -3.517624 \\
\hline & 2.470779 & 3508 & -1.792252 \\
\hline & 2.632645 & 3.6 & 0.806656 \\
\hline & -4.580719 & -2.531631 & 1.917740 \\
\hline & 2.857849 & 0.237915 & -2.992722 \\
\hline & -2.465027 & 322 & 1.308197 \\
\hline & -1.933380 & 6.2 & 2.4 \\
\hline & 2.527954 & -3.879974 & 0.001343 \\
\hline & -1.730667 & -6.531021 & 1.400787 \\
\hline & -1.721542 & 5.876724 & -0.30317 \\
\hline & 3.254257 & -1.621719 & -1.081985 \\
\hline & -0.533081 & -1.716431 & -6.32189 \\
\hline & 4.448685 & 2.535660 & -3.207336 \\
\hline & 4.786514 & 1.996185 & 1.732498 \\
\hline & 0.012985 & 1.887146 & 5.07212 \\
\hline & -1.736084 & 6.531006 & -3.10333 \\
\hline & -4.740128 & 2.605301 & $-3.1687 €$ \\
\hline & 0.630188 & 5.622925 & 1.089905 \\
\hline & -0.116394 & -2.960220 & 4.2912 \\
\hline & & 70 & \\
\hline
\end{tabular}




$\begin{array}{rrrr}\mathrm{O} & -1.965576 & -5.919678 & -4.190369 \\ \mathrm{O} & -0.468491 & 2.747864 & -5.895645 \\ \mathrm{O} & 4.595322 & -2.527328 & 1.307541 \\ \mathrm{O} & 3.046844 & -6.227264 & -1.596710 \\ \mathrm{O} & 4.417969 & -1.963852 & -3.704483 \\ \mathrm{O} & 3.110565 & 6.266251 & -0.220764 \\ \mathrm{C} & -0.673615 & 3.230484 & 5.310242 \\ \mathrm{C} & -0.309769 & 4.326035 & 4.286057 \\ \mathrm{O} & -0.087585 & 5.477829 & 4.641464 \\ \mathrm{H} & 1.067398 & 1.880112 & 5.423691 \\ \mathrm{H} & -0.529190 & 1.030350 & 5.524246 \\ \mathrm{H} & -1.777466 & 3.098404 & 5.213150 \\ \mathrm{H} & -0.477280 & 3.660294 & 6.321899\end{array}$

\subsection{2 $\underline{\text { Conf2 }}$}

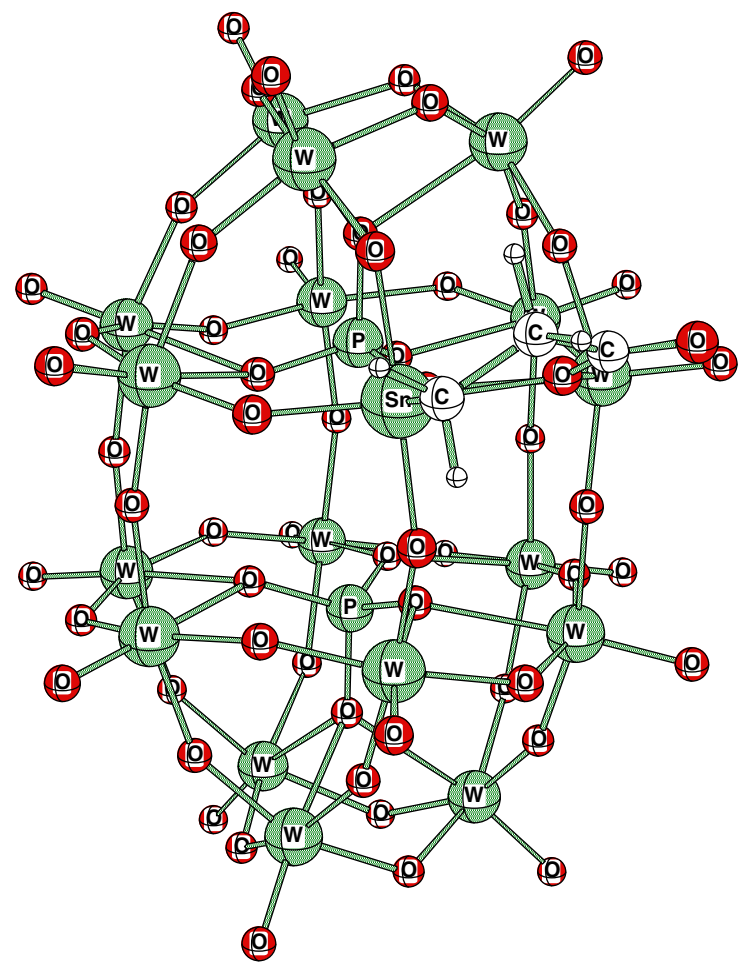

89

\begin{tabular}{|c|c|c|c|}
\hline \multicolumn{4}{|c|}{ Energy $=-6609.8913414360$} \\
\hline Sn & -0.184110 & & 0.0230 \\
\hline & 927 & 8691 & \\
\hline & 1.646217 & 0.925114 & 43306 \\
\hline & 2.677 & 0.1 & \\
\hline b & -0.499286 & 2. & \\
\hline N & -3.7 & 070 & \\
\hline & -4.2 & 072 & \\
\hline N & -4.2 & -2 & -5 \\
\hline$N$ & -1.324288 & 1.727667 & -6.8 \\
\hline$N$ & 1. & 18 & \\
\hline N & -3.7 & -3.2 & \\
\hline N & 487 & 4890 & -2.1 \\
\hline$N$ & 1.900608 & 9045 & -6.4 \\
\hline$N$ & 2.43 & -3.2 & \\
\hline$N$ & -0.2 & & \\
\hline W & -2.2 & -5.4 & -6. \\
\hline & & & \\
\hline
\end{tabular}

\begin{tabular}{|c|c|c|c|}
\hline & & & \\
\hline & -1.511637 & 3.495377 & -0.699821 \\
\hline & 1.013415 & -5.841624 & \\
\hline & -1.695995 & -6.280011 & $-3.1181 \varepsilon$ \\
\hline & -3.387703 & 3.023818 & -4.080388 \\
\hline & 2.551194 & -1.426917 & -2.1294 \\
\hline & -1.366667 & 3.320658 & -5.6824 \\
\hline & 2.744676 & 0.618628 & -3.98179 \\
\hline & -2.133005 & -3.323331 & -1.01167 \\
\hline & -2.226218 & 0.503473 & -3.027457 \\
\hline & -4.417803 & -0.615078 & $-4.76107 \varepsilon$ \\
\hline & -2.224215 & -0.124732 & -0.075223 \\
\hline & 1.637613 & -3.938404 & -1.219931 \\
\hline & -3.986961 & -1.397212 & -1.663770 \\
\hline & 0.081273 & -2.832350 & -2.980965 \\
\hline & -3.125030 & -5.054069 & -2.71693 \\
\hline & 0.632751 & 1.979604 & -6.920672 \\
\hline & -2.505070 & -6.586376 & -4.908807 \\
\hline & -4.752295 & 1.087465 & -2.617305 \\
\hline & -1.095636 & -0.085866 & -7.397946 \\
\hline & 0.074684 & $-1.9 s$ & 4790 \\
\hline & 2.112346 & 0.409490 & -0.057313 \\
\hline & -4.854527 & -3.113826 & -3.660 \\
\hline & -2.352886 & -2.539136 & -3.882576 \\
\hline & 0.230448 & 0.277110 & 2875 \\
\hline & 0.601223 & -2.203140 & -7.903771 \\
\hline & 1.516953 & -4.257487 & -6.286395 \\
\hline & -2.994312 & -1.852143 & -6.721210 \\
\hline & -0.317954 & -2.199728 & -5.48992 \\
\hline & -0.001929 & -6.871162 & -3.96836 \\
\hline & -0.682164 & -5.362571 & -1.788599 \\
\hline & -0.404384 & -6.2 & -6 \\
\hline & -3.601697 & -4.247666 & -5.86282 \\
\hline $\mathrm{O}$ & -0.708704 & 2.555075 & -2.824018 \\
\hline & -1.550399 & -3.9 & 5846 \\
\hline & -0.748819 & 0.983680 & -3.194667 \\
\hline & -0.874124 & -2.956194 & -4.226146 \\
\hline & -0.059508 & 4.8 & 302 \\
\hline & -0.17 & 0.8 & -4.6 \\
\hline & -4.961426 & 0.539503 & 0.16069 \\
\hline & -5.765060 & -2.531291 & -6.285008 \\
\hline & -0.921245 & -4.5 & 172 \\
\hline & -2.960888 & 1.2 & -5 \\
\hline & 1.707938 & 2.954316 & -4.501002 \\
\hline & 2.162514 & 2.332670 & -2.010622 \\
\hline & -4.937577 & -3.91 & \\
\hline & 1.900166 & $-0.4 C$ & -6.2 \\
\hline & -2.997166 & 2.307093 & -0.88124 \\
\hline & -1.949884 & 4.52 & 905 \\
\hline & 1.922162 & -4.905121 & $-3.8055 t$ \\
\hline & -2.183248 & -7.758441 & -2.303 \\
\hline & -2.190292 & 4.611429 & -2.211086 \\
\hline & 2.506352 & -2.476249 & -4.617066 \\
\hline & -1.815556 & -1.931275 & -9.37567 \\
\hline & 3.399385 & 1.963705 & -6.49209 \\
\hline & 4.457427 & 0.637758 & -1.795348 \\
\hline & -2.630402 & 5.756610 & -4.78032 \\
\hline & -5.694669 & 1.858018 & -5.161608 \\
\hline & 0.285632 & 4.319112 & -1.23405 \\
\hline & -0.250120 & -4.550742 & 0.8 \\
\hline
\end{tabular}




$\begin{array}{rrrr}\text { W } & 1.287987 & 3.904155 & -2.799688 \\ \mathrm{O} & -3.038620 & -6.394741 & -7.708893 \\ \mathrm{O} & -1.842119 & 2.456748 & -8.321435 \\ \mathrm{O} & 4.107727 & -3.722140 & -2.551953 \\ \mathrm{O} & 2.212166 & -7.022086 & -5.775446 \\ \mathrm{O} & 3.381440 & -2.494439 & -7.373710 \\ \mathrm{O} & 2.559152 & 5.110950 & -2.710817 \\ \mathrm{O} & 3.878606 & 0.848304 & 1.306571 \\ \mathrm{H} & 0.575318 & -1.000696 & 2.414956 \\ \mathrm{H} & -0.456326 & 0.464963 & 2.727808 \\ \mathrm{H} & 1.351182 & 1.998084 & 2.161050 \\ \mathrm{H} & 2.204405 & 0.807140 & 3.203714\end{array}$

\subsection{3 $\underline{\text { Conf3 }}$}

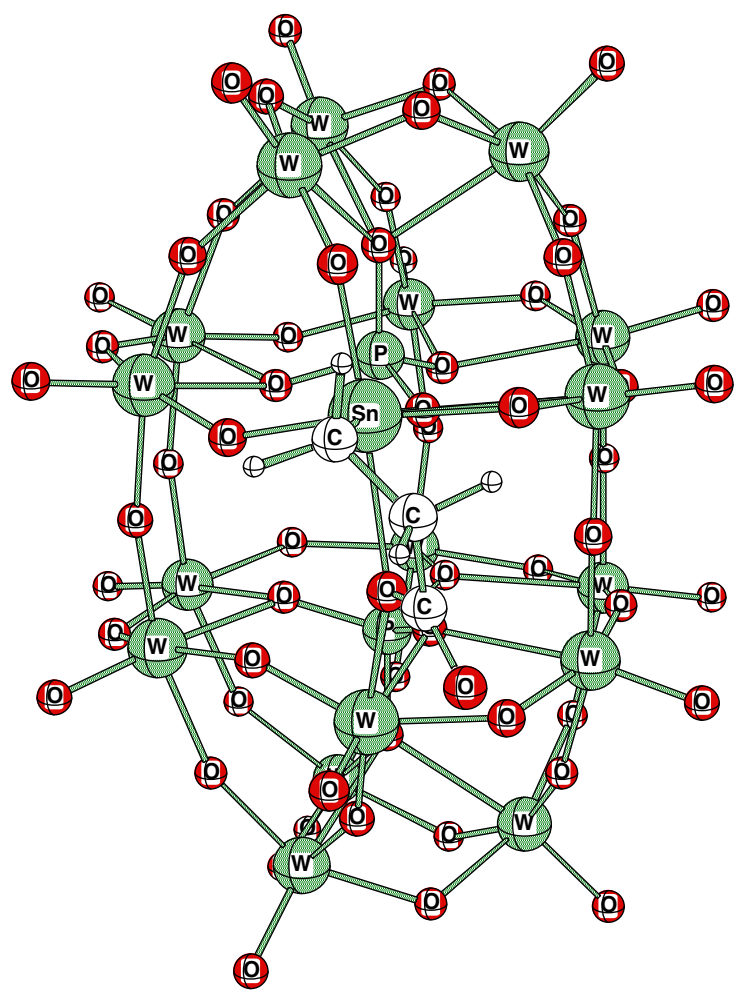

89

\begin{tabular}{|c|c|c|c|}
\hline \multicolumn{4}{|c|}{ Energy $=-6609.8834334870$} \\
\hline Sn & -0.288757 & 1.064453 & 3.06115 \\
\hline & -0.671082 & 0.719986 & 5.17726 \\
\hline C & 0.362244 & -0.311340 & 5.603622 \\
\hline & 0.509949 & -1.507843 & 4.637421 \\
\hline J & 1.800873 & 73 & 3.5 \\
\hline U & -0.657959 & 3.115906 & 3.114029 \\
\hline$N$ & -3.3 & & \\
\hline$N$ & -3.0 & & \\
\hline$N$ & -2.980133 & -1.3 & -3. \\
\hline$N$ & 0.361343 & 2.857269 & -3.813416 \\
\hline N & 3.1 & 7 & 569 \\
\hline $\mathrm{VV}$ & -3.26 & -2.1 & 0.0 \\
\hline W & 2.923676 & 1.350601 & 1.827515 \\
\hline W & 3.240143 & -1.389740 & -2.904526 \\
\hline W & 2.88 & -2.3 & 0.7 \\
\hline VV & -0.26 & -3.1 & 2.0 \\
\hline W & -0.72 & -4.3 & -4.085938 \\
\hline W & -0.95 & 5.357 & -1.3030 \\
\hline
\end{tabular}

\begin{tabular}{|c|c|c|c|}
\hline & & & \\
\hline & 3619 & 210 & 2.083557 \\
\hline & 2.034943 & -4.859848 & -2.00001 \\
\hline & -1.201218 & -5.354446 & -0.81973 \\
\hline & -2.367828 & 4.084244 & -1.70140 \\
\hline & 2.881592 & -0.592957 & 1.500671 \\
\hline & 0.007690 & 4.414902 & 600 \\
\hline & 3.348480 & 1.531952 & -0.07333 \\
\hline & -1.851074 & -2.309265 & 1.41378 \\
\hline & -1.575745 & 1.567657 & -0.51100 \\
\hline & -3.224396 & 0.449707 & \\
\hline & -2.176239 & 0.783722 & 2.290100 \\
\hline & 1.668900 & -3.087891 & 2.080704 \\
\hline & -3.542862 & -0.373184 & 0.367752 \\
\hline & 0.629730 & -1.9 & 0.04 \\
\hline & -2.512390 & -4.019211 & -0.513550 \\
\hline & 2.254929 & 2.972641 & -3.410110 \\
\hline & -1.536285 & -5.530838 & -2.682800 \\
\hline & -4.074371 & 2.121689 & -0.686646 \\
\hline & 0.644150 & 1.016891 & -4.419037 \\
\hline & 0.025879 & -1.312424 & 3.400757 \\
\hline & -3.963776 & -2.060806 & -1.803070 \\
\hline & -1.487991 & -1.510849 & -1.422394 \\
\hline & 0.518723 & 1.182724 & 1.010345 \\
\hline & 2.337814 & 9261 & -4.609650 \\
\hline & 2.785843 & -3.301941 & -2.888947 \\
\hline & -1.423386 & -0.741791 & -4.334152 \\
\hline & 0.876343 & -1.199158 & -2.470200 \\
\hline & 0.651245 & 230 & -1.121841 \\
\hline & -0.405167 & -4.556366 & 0.879242 \\
\hline & 0.956711 & -5.1 & -3.662750 \\
\hline & -2.230743 & 052 & -3.724243 \\
\hline & -0.092102 & 3.539276 & 0.183411 \\
\hline & 0.141373 & -2.795334 & -4.799133 \\
\hline & $-0.0 \varepsilon$ & 36 & 0015 \\
\hline & 0.016571 & -1.97 & -1.409042 \\
\hline & 0.778397 & 5.892792 & -0.583603 \\
\hline & 0.822037 & 1.840332 & -1.510880 \\
\hline & -4.8 & 95 & \\
\hline & -4.244492 & -1.40 & 979 \\
\hline & 0.022064 & -3.535110 & -1.872101 \\
\hline & -1.461212 & 2.355194 & -3.436966 \\
\hline & 2.6 & 3.9 & -0.851227 \\
\hline & 2.455338 & 3.27 & 1.670380 \\
\hline & -4.656555 & -2.859406 & 0.822495 \\
\hline & 3.233795 & 0.521606 & -2.587479 \\
\hline & -2.75 & & 1.489670 \\
\hline & -2.088348 & 5.5 & 3.291214 \\
\hline & 2.522354 & -4.006348 & -0.370361 \\
\hline & -1.934998 & -6.824524 & -0.2000 \\
\hline & -1.691910 & 5.61 & 0.461548 \\
\hline & 3.398788 & -1.620667 & -0.974731 \\
\hline & 0.356491 & -0.678513 & -6.608505 \\
\hline & 4.824448 & 2.786377 & -2.20225 \\
\hline & 4.489532 & 1.50 & $2.590 \varsigma$ \\
\hline & -1.472900 & 6.824524 & -2.11256 \\
\hline & -4.291290 & 2.955490 & $-3.3883 C$ \\
\hline & 0.495407 & 5.287079 & 2.000916 \\
\hline & -0.767273 & -3.993134 & 3.4901 \\
\hline & 1.883 & 4.835800 & 0.675 \\
\hline
\end{tabular}




$\begin{array}{rrrr}\mathrm{O} & -1.211472 & -5.209747 & -5.524307 \\ \mathrm{O} & 0.272980 & 3.625153 & -5.381973 \\ \mathrm{O} & 4.408966 & -2.916382 & 1.376465 \\ \mathrm{O} & 3.280823 & -6.076523 & -2.217484 \\ \mathrm{O} & 4.892456 & -1.558655 & -3.459427 \\ \mathrm{O} & 3.100189 & 6.026749 & 1.102249 \\ \mathrm{O} & 1.041260 & -2.534851 & 5.038605 \\ \mathrm{H} & -1.716293 & 0.351349 & 5.258194 \\ \mathrm{H} & -0.573090 & 1.694534 & 5.700989 \\ \mathrm{H} & 1.369156 & 0.162537 & 5.646103 \\ \mathrm{H} & 0.175995 & -0.764847 & 6.608505\end{array}$

\subsubsection{Conf4}

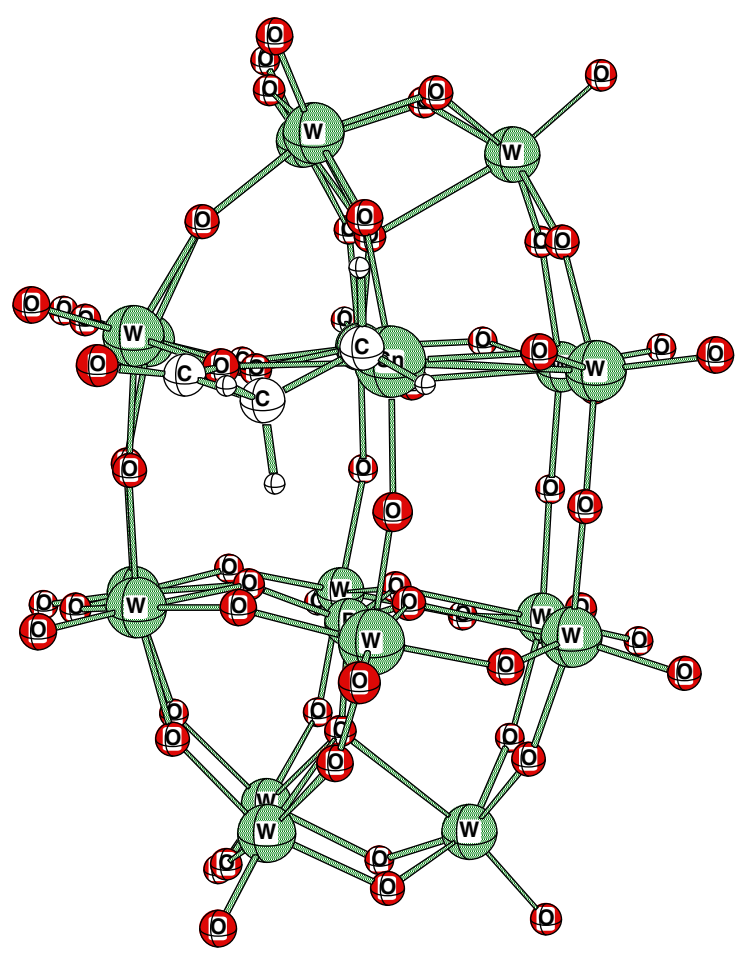

89

Energy $=-6609.8835067750$

$\begin{array}{crrr}\text { O } & -2.113446 & -0.160604 & 0.138929 \\ \text { Sn } & 0.242891 & -0.015291 & -0.009330 \\ \text { C } & -0.076821 & -2.172139 & -0.034399 \\ \text { C } & -1.467867 & -2.372554 & -0.632265 \\ \text { C } & -2.541654 & -1.416901 & -0.077234 \\ \text { O } & 2.323084 & 0.117022 & -0.059441 \\ \text { O } & 0.062048 & 0.149961 & 2.072877 \\ \text { W } & -3.410738 & 1.535743 & 0.902257 \\ \text { W } & -3.475884 & 4.993278 & 1.246977 \\ \text { W } & -4.072319 & 5.357391 & -2.530540 \\ \text { W } & -0.345852 & 7.055532 & 1.080651 \\ \text { W } & 2.702616 & 5.553183 & 0.287721 \\ \text { W } & -3.758375 & 1.909010 & -2.958042 \\ \text { W } & 3.116904 & 1.816624 & -0.212359 \\ \text { W } & 2.057120 & 6.147792 & -3.458818 \\ \text { W } & 2.406315 & 2.427530 & -3.958156 \\ \text { W } & -0.406677 & 0.393669 & -3.847045 \\ \text { W } & -2.397387 & 6.047479 & -5.867452 \\ \text { W } & -0.855166 & 4.638568 & 3.972463 \\ \text { W } & -1.017928 & 7.548795 & -2.667191\end{array}$

\begin{tabular}{|c|c|c|c|}
\hline & 309 & 405 & \\
\hline & 8979 & 9731 & -6.589449 \\
\hline & -2.230801 & 2.604492 & 4215 \\
\hline & -2.480372 & 4.641630 & 2.907647 \\
\hline & 2.772719 & 1.891916 & -2.134055 \\
\hline & -0.324577 & 6.088138 & 2.768215 \\
\hline & 3.131842 & 3.774712 & -0.273605 \\
\hline & -2.226566 & 0.803886 & -3.17484 \\
\hline & -1.801801 & 3.303460 & 0.446426 \\
\hline & -3.971059 & 5.005322 & -0.595374 \\
\hline & 1.432106 & 0.790284 & -4.354596 \\
\hline & -3.674830 & 1.735701 & -0.999675 \\
\hline & 0.124968 & 2.722389 & -3.233949 \\
\hline & -3.442502 & 2.381493 & -4.869631 \\
\hline & 1.570520 & 7.00 & 0.971182 \\
\hline & -2.971022 & 4.387021 & -6.727432 \\
\hline & -4.232973 & 3.155152 & 1.405702 \\
\hline & -0.474493 & 7.435433 & -0.813716 \\
\hline & 0.071099 & 0.027774 & -2.093089 \\
\hline & -4.781838 & 3.577232 & -2.797573 \\
\hline & -2.212256 & 3.7 & -2.669819 \\
\hline & 0.473189 & 2.1 & -0.101990 \\
\hline & 0.852965 & 7.654235 & -3.223439 \\
\hline & 1.398158 & 5.786434 & -5.240206 \\
\hline & -2.680113 & 6.682146 & -2.213446 \\
\hline & -0.114483 & 5.296717 & -2.829025 \\
\hline & -0.597024 & 3.265318 & -7.297727 \\
\hline & -1.096943 & 1.326987 & -5.467132 \\
\hline & -0.755530 & 5.922423 & -6.885337 \\
\hline & -3.639683 & 5.602026 & -4.401225 \\
\hline & 0.056576 & 3.1 & 2.217761 \\
\hline & -1.530502 & & -4.526628 \\
\hline & -0.248122 & 3.370262 & 0.644616 \\
\hline & -0.810175 & 3.983158 & -3.346754 \\
\hline & 1.0 & 4.3 & 4.333262 \\
\hline & 0.376943 & 4.74 & 0.234877 \\
\hline & -4.738620 & 0.503805 & 1.349521 \\
\hline & -5.545543 & 6.300877 & -2.512403 \\
\hline & -1.0 & 4.2 & 535 \\
\hline & -2.163865 & 6.3 & 996 \\
\hline & 2.579221 & 4.725588 & 2.109557 \\
\hline & 2.827145 & 2.138445 & 1.728452 \\
\hline & -5.072139 & 0.7 & 3081 \\
\hline & 2.363935 & 6.087429 & -1.510820 \\
\hline & -2.277428 & 1.329828 & 2.415515 \\
\hline & -0.945356 & -0.038152 & 4.712054 \\
\hline & 1.63 & 3.2 & -5.634710 \\
\hline & -2.984069 & 1.671587 & -7.625397 \\
\hline & -1.202420 & 2.795678 & 4.469579 \\
\hline & 2.622735 & 4.28 & -3.463344 \\
\hline & -1.453262 & 9.243899 & -2.699069 \\
\hline & 4.279303 & 6.277127 & 0.509277 \\
\hline & 4.814227 & 1.400858 & -0.198833 \\
\hline & -1.336897 & 5.496595 & 5.425316 \\
\hline & -4.803914 & 5.902628 & 1.932828 \\
\hline & 1.231764 & 1.682541 & 4.002382 \\
\hline & -0.571421 & -1.239392 & -4.472323 \\
\hline & 2.256371 & 3.217195 & 3.229904 \\
\hline & -3.289341 & 7.233357 & -6.805541 \\
\hline & -0.6017 & 8.665467 & 1.71 \\
\hline
\end{tabular}




$\begin{array}{rrrr}\mathrm{O} & 3.962721 & 2.018392 & -4.648455 \\ \mathrm{O} & 1.673892 & 4.856480 & -7.989471 \\ \mathrm{O} & 3.516369 & 7.006981 & -3.904098 \\ \mathrm{O} & 3.666308 & 3.184405 & 4.273654 \\ \mathrm{O} & -3.682788 & -1.812035 & 0.118572 \\ \mathrm{H} & -0.002998 & -2.529728 & 1.015798 \\ \mathrm{H} & 0.724801 & -2.630336 & -0.651516 \\ \mathrm{H} & -1.426194 & -2.188198 & -1.731227 \\ \mathrm{H} & -1.877126 & -3.400674 & -0.487432\end{array}$

\subsection{Cyclization : Keggin Type}

\subsection{1 $\underline{\text { Reagent }}$}

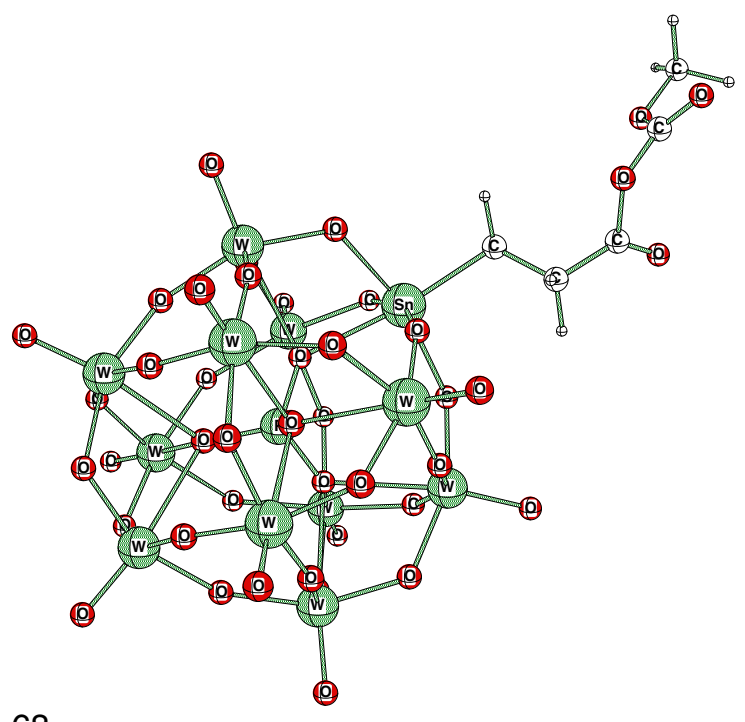

68

\begin{tabular}{|c|c|c|c|}
\hline \multicolumn{4}{|c|}{ Energy $=-4513.8390841620$} \\
\hline & -2.123184 & -1.481064 & 84 \\
\hline & -1.829590 & 392776 & 150 \\
\hline & -1.250900 & -2.620560 & -0.5 \\
\hline & -3 & 37 & \\
\hline & -1.729874 & -0.090240 & -0.5661 \\
\hline$N$ & -2.898666 & .188644 & 3.24728 \\
\hline V & -1.252258 & -3.275696 & \\
\hline & -4.8 & -3 & \\
\hline & -2.0 & 385 & -3 \\
\hline & & -2.7 & \\
\hline v & -5.5 & -0 . & \\
\hline N & -2.208191 & -3.2 & 3. \\
\hline W & -2.853302 & 003 & -0. \\
\hline W & -4.7 & 24 & \\
\hline 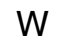 & 0.4 & -0 & \\
\hline W & -1.3 & -5.0 & 0.0 \\
\hline Sn & & 91 & -0 \\
\hline & -4.5 & & \\
\hline $\mathrm{O}$ & -3.73 & -0.55 & -2.827 \\
\hline 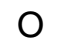 & -1.445053 & -4.412491 & 1.927 \\
\hline & -3.19 & -3.1 & -2.792 \\
\hline & 0.62637 & 0.420 & 1.437 \\
\hline O & -2.6 & & \\
\hline & -3.2841 & -4.81275 & 0.1201 \\
\hline & -4.5 & & \\
\hline
\end{tabular}

$\begin{array}{rrrr}\mathrm{O} & -1.308670 & -1.368515 & -3.279526 \\ \mathrm{O} & 1.253632 & -0.922501 & -0.959824 \\ \mathrm{O} & -3.879059 & -3.562531 & 2.337433 \\ \mathrm{O} & 1.065262 & -2.219650 & 1.372162 \\ \mathrm{O} & -0.469757 & -2.493362 & 3.613647 \\ \mathrm{O} & 0.496613 & -4.513596 & 0.004959 \\ \mathrm{O} & -5.899231 & -0.952881 & -1.196411 \\ \mathrm{O} & -0.992264 & 2.464676 & -0.085556 \\ \mathrm{O} & -1.396896 & -4.906677 & -1.843460 \\ \mathrm{O} & -5.338013 & -3.561234 & -1.159424 \\ \mathrm{O} & -0.989365 & 0.125809 & 3.598694 \\ \mathrm{O} & -0.328568 & 1.080536 & -2.561188 \\ \mathrm{O} & -5.946411 & -2.298248 & 1.109406 \\ \mathrm{O} & -2.963882 & -1.619843 & 4.023895 \\ \mathrm{O} & 0.582733 & -3.178421 & -2.304855 \\ \mathrm{O} & -2.867325 & 1.868729 & -1.972122 \\ \mathrm{O} & -3.462173 & 1.162369 & 4.582626 \\ \mathrm{O} & -1.029297 & -3.947769 & -4.532089 \\ \mathrm{O} & -5.965271 & -5.179565 & 1.136963 \\ \mathrm{O} & -2.193283 & 1.057114 & -4.675552 \\ \mathrm{O} & 2.956329 & -3.162918 & -0.631439 \\ \mathrm{O} & -7.104614 & 0.335312 & 1.076920 \\ \mathrm{O} & -2.367493 & -4.355530 & 4.675568 \\ \mathrm{O} & -3.489975 & 3.751587 & 0.140549 \\ \mathrm{O} & -5.825668 & -2.326263 & -3.720535 \\ \mathrm{O} & 1.789444 & -0.719910 & 3.756332 \\ \mathrm{O} & -1.189316 & -6.806335 & 0.299942 \\ \mathrm{C} & 2.218475 & 2.143494 & -0.810593 \\ \mathrm{C} & 3.435913 & 1.477341 & -0.126587 \\ \mathrm{H} & 2.375977 & 2.240341 & -1.906174 \\ \mathrm{H} & 2.010620 & 3.149673 & -0.388275 \\ \mathrm{C} & 4.688843 & 2.241852 & -0.425827 \\ \mathrm{H} & 3.271805 & 1.431900 & 0.972031 \\ \mathrm{H} & 3.577591 & 0.442490 & -0.500702 \\ \mathrm{O} & 5.588852 & 2.002319 & -1.190598 \\ \mathrm{O} & 4.748886 & 3.458511 & 0.372223 \\ \mathrm{C} & 5.509018 & 4.493454 & -0.038300 \\ \mathrm{O} & 6.288147 & 5.103180 & 0.667252 \\ \mathrm{O} & 5.234573 & 4.812073 & -1.329193 \\ \mathrm{C} & 6.023712 & 5.860687 & -1.875580 \\ \mathrm{H} & 5.671265 & 5.989578 & -2.919052 \\ \mathrm{H} & 5.891708 & 6.806320 & -1.303635 \\ \mathrm{H} & 7.104614 & 5.592834 & -1.867752 \\ & & & \end{array}$

\subsubsection{Transition State}




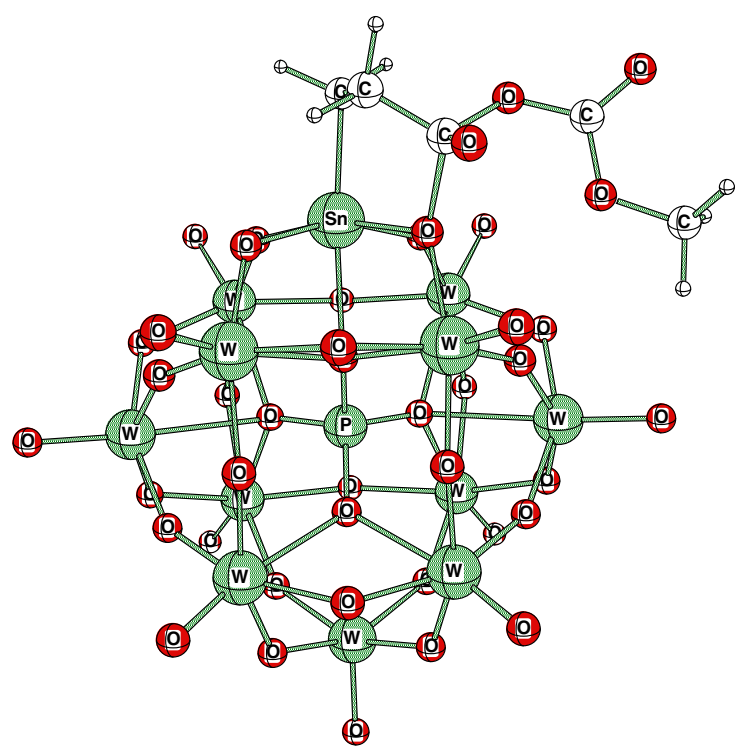

68

Energy $=-4513.8168014710$

Sn $-0.536972 \quad 2.851990$

C $1.230087 \quad 4.101456 \quad 3.220123$

$\begin{array}{llll}\text { C } & -0.058319 & 4.872299 & 2.871002\end{array}$

$\begin{array}{llll}\text { C } & -0.458237 & 4.927521 & 1.392456\end{array}$

$\begin{array}{llll}\mathrm{P} & -0.400833 & -0.603745 & -0.036972\end{array}$

$\begin{array}{llll}\text { O } & 0.879868 & -0.395309 & -0.926178\end{array}$

$\begin{array}{llll}0 & -1.692017 & -0.465851 & -0.923416\end{array}$

$\begin{array}{llll}\mathrm{O} & -0.363403 & -2.029816 & 0.623657\end{array}$

$\begin{array}{llll}O & -0.424622 & 0.529968 & 1.093613\end{array}$

W $3.217285-0.483414 \quad-0.109344$

W $-4.045105 \quad-0.753174 \quad-0.165909$

W $\quad-0.286530 \quad-4.264282 \quad-0.710312$

W $\quad-2.258728 \quad 0.631714 \quad 2.902100$

W $\quad-2.357025 \quad 1.553787 \quad-2.267502$

W $1.408905 \quad-2.971512 \quad 2.100815$

W $1.559219 \quad-1.806427 \quad-2.918564$

W $\quad 1.298874 \quad 0.723190 \quad 2.946152$

W $\quad-2.109192 \quad-3.078445 \quad 2.083206$

W $1.406845 \quad 1.676910-2.249115$

W $\quad-2.236328 \quad-1.929916 \quad-2.960419$

O $1.264038 \quad-1.229904 \quad 2.939850$

$\begin{array}{llll}\mathrm{O} & -2.085968 & -1.348053 & 2.912018\end{array}$

$\begin{array}{llll}0 & -0.340424 & -1.733780 & -3.374557\end{array}$

$\begin{array}{llll}O & -3.359955 & -2.303375 & 0.820099\end{array}$

$\begin{array}{llll}\mathrm{O} & 0.891434 & 2.632080 & -0.720001\end{array}$

$\begin{array}{llll}O & 2.515854 & 0.489426 & 1.426926\end{array}$

$\begin{array}{llll}\mathrm{O} & -1.610336 & -3.417007 & -1.836212\end{array}$

$\begin{array}{llll}O & 2.624954 & -2.066483 & 0.881989\end{array}$

$\begin{array}{llll}\mathrm{O} & -3.474594 & 0.245972 & 1.390823\end{array}$

$\begin{array}{lllll}\mathrm{O} & -1.924683 & 2.576172 & -0.749680\end{array}$

$\begin{array}{llll}\text { O } & 1.007416 & -3.322678 & -1.804764\end{array}$

$\begin{array}{llll}\mathrm{O} & -0.471222 & 1.590530 & -2.773438\end{array}$

$\begin{array}{llll}\text { O } & 1.720718 & 0.057938 & -3.371933\end{array}$

$\begin{array}{llll}\text { O } & -2.525589 & -0.081985 & -3.400864\end{array}$

$\begin{array}{llll}\text { O } & -0.335266 & -3.442291 & 2.843033\end{array}$

$\begin{array}{llll}\text { O } & -3.801010 & -1.807175 & -1.808578\end{array}$

$\begin{array}{llll}O & -1.611450 & -4.394318 & 0.707520\end{array}$

$\begin{array}{llll}\text { O } & 3.017960 & 1.056656 & -1.264053\end{array}$ $\begin{array}{llll}\text { O } & -2.008240 & 2.367630 & 2.247391\end{array}$

$\begin{array}{llll}\text { O } & 1.049026 & -4.303772 & 0.721542\end{array}$

$\begin{array}{llll}\text { O } & 3.096436 & -1.568192 & -1.744492\end{array}$

$\begin{array}{llll}\mathrm{O} & -3.923431 & 0.814331 & -1.308380\end{array}$

$\begin{array}{llll}\mathrm{O} & -0.480286 & 0.690018 & 3.738663\end{array}$

$\begin{array}{lllll}O & 4.937103 & -0.475266 & 0.190033\end{array}$

$\begin{array}{llll}O & -5.768845 & -0.885452 & 0.069183\end{array}$

$\begin{array}{llll}\text { O } & -0.223267 & -5.898849 & -1.318268\end{array}$

$\begin{array}{llll}\mathrm{O} & -3.334244 & 0.905167 & 4.248703\end{array}$

$\begin{array}{llll}\text { O } & -3.094818 & 2.734299 & -3.322540\end{array}$

$\begin{array}{llll}\text { O } & 2.486267 & -3.842484 & 3.162689\end{array}$

$\begin{array}{llll}\text { O } & 2.275635 & -2.588242 & -4.305054\end{array}$

$\begin{array}{llll}\text { O } & 2.329773 & 1.000824 & 4.312805\end{array}$

$\begin{array}{llll}\text { O } & -3.141724 & -4.024582 & 3.126282\end{array}$

$\begin{array}{llll}\text { O } & 2.060226 & 2.920227 & -3.285934\end{array}$

$\begin{array}{llll}O & -2.869614 & -2.751389 & -4.364273\end{array}$

H $\quad 0.293488 \quad 5.459946 \quad 0.775314$

H $\quad-1.462494 \quad 5.381989 \quad 1.250763$

H $\quad 0.111496 \quad 5.898849 \quad 3.279938$

$\begin{array}{llll}\mathrm{H} & -0.865250 & 4.402924 & 3.473404\end{array}$

$\begin{array}{lllll}\mathrm{O} & 1.593765 & 3.908371 & 4.364273\end{array}$

$\begin{array}{llll}\text { O } & 2.280411 & 4.785858 & 2.259888\end{array}$

C $3.568878 \quad 4.471863 \quad 2.268906$

$\begin{array}{llll}\text { O } & 4.467606 & 5.268555 & 2.014008\end{array}$

$\begin{array}{llll}\text { O } & 3.787247 & 3.169586 & 2.565262\end{array}$

C $5.1328892 .726135 \quad 2.505569$

$\begin{array}{llll}\text { H } & 5.100037 & 1.643143 & 2.734833\end{array}$

H $\quad 5.558777 \quad 2.881241 \quad 1.489929$

H $\quad 5.768860 \quad 3.266663 \quad 3.246445$

\section{${ }_{1.4}$ Cyclization : Dawson $\alpha_{1}$}

\subsubsection{Reagent}

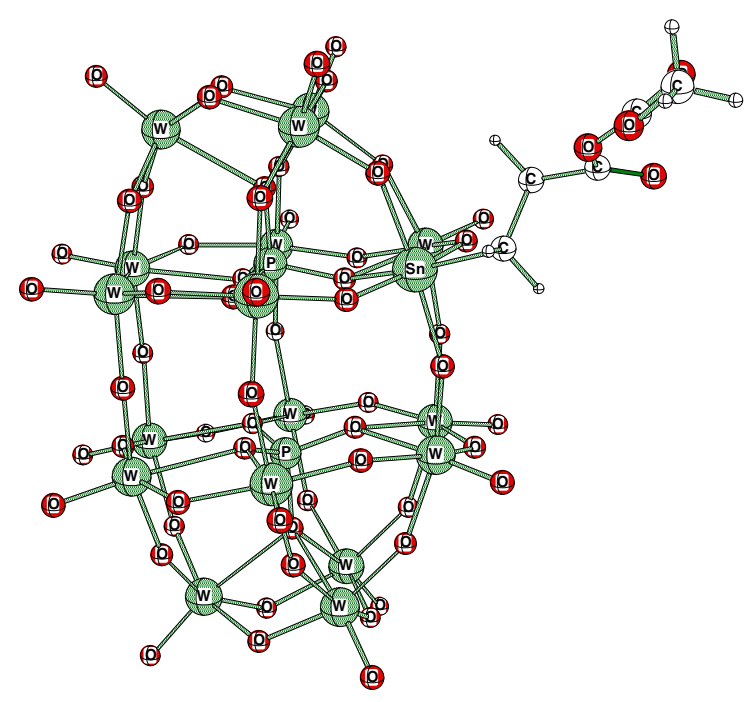

97

Energy $=-6913.0872964490$

$\begin{array}{lrrr}\text { W } & -2.218185 & 1.149139 & 2.109558 \\ \text { W } & -5.185730 & 1.231064 & 0.236481 \\ \text { W } & -4.887390 & -2.547729 & -0.447067 \\ \text { W } & -5.098663 & 1.860275 & -3.483475 \\ \text { W } & -2.024017 & 2.420273 & -5.047638\end{array}$ 


\begin{tabular}{|c|c|c|c|}
\hline & 37 & 11 & \\
\hline & 1.255692 & 2.341019 & مी \\
\hline & 31491 & 892 & \\
\hline & 3129 & 3099 & \\
\hline & 0.972885 & 1.734467 & 886 \\
\hline & 1316 & -2.092896 & \\
\hline & -3.524475 & -5.216766 & -2.7904 \\
\hline & -4.319275 & 4.699570 & -1.082 \\
\hline & -4.780411 & -1.910568 & \\
\hline & -1.385635 & & \\
\hline & -0.468735 & -4.675827 & -4.375702 \\
\hline & -0.568008 & -5.285141 & -0.937744 \\
\hline & -4.90 & 3.15 & \\
\hline & 977 & 49 & \\
\hline & -4.817642 & & -2 \\
\hline & -0.226608 & 2.12 & -4.414215 \\
\hline & -0.1 & -2.1 & \\
\hline & -2.77 & 1.1 & -0. \\
\hline & -5.032745 & -0.708755 & 0. \\
\hline & -0.4 & & \\
\hline & 2.290955 & -1.985825 & $-2 . c$ \\
\hline b & -2.3 & -0.7 & \\
\hline & -0.2 & -1.5 & -2 \\
\hline & -1.5 & -4.5 & \\
\hline b & -3.97 & 2.4 & -4. \\
\hline 0 & -2.2 & -6.0 & \\
\hline 0 & -4.1 & & \\
\hline b & -4.9 & 0.0 & -4 \\
\hline 0 & 1.5 & -0.2 & $-0 .($ \\
\hline b & 2.0 & & \\
\hline & -3.8 & -2.9 & \\
\hline b & -2.5 & -1.9 & -0 . \\
\hline 0 & -0.47 & & -1.4 \\
\hline 0 & -3.6 & -1.7 & \\
\hline b & -1.3 & -3 & \\
\hline 0 & $-5.2 C$ & $-1 . s$ & -2 \\
\hline 0 & -2.4 & -1.5 & -3 \\
\hline 0 & & & \\
\hline D & 0.22 & & \\
\hline b & $0.7 \varepsilon$ & -3.9 & -0 \\
\hline 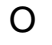 & -2.2 & & \\
\hline b & -4.3 & -4.2 & \\
\hline 0 & -2.1 & & -1 . \\
\hline 0 & -4.23 & -3.7 & -3 \\
\hline 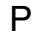 & -2.0 & & \\
\hline & -1.7 & & \\
\hline 0 & -3.1 & & -2.2 \\
\hline 0 & -2.6 & 1.6 & $-2 . \varepsilon$ \\
\hline ก & -2.1 & & \\
\hline O & -6.4 & -3. & \\
\hline $\mathrm{O}$ & -1.6 & -3.7 & -2. \\
\hline $\mathrm{O}$ & $-5.4<$ & & -1.7 \\
\hline O & -1.9 & & \\
\hline Q & & & \\
\hline $\mathrm{O}$ & $-1.7 t$ & -3.2 & \\
\hline O & -2.0 & & -5. \\
\hline $\mathrm{O}$ & -2.15 & & \\
\hline 0 & & & \\
\hline & & & \\
\hline & & & \\
\hline
\end{tabular}

$\begin{array}{rrrr}\mathrm{O} & -3.233047 & 5.257126 & 0.415909 \\ \mathrm{O} & 0.028275 & -1.079819 & -4.973145 \\ \mathrm{O} & -6.320587 & -2.248383 & -4.932236 \\ \mathrm{O} & -1.779388 & 3.076263 & -6.656891 \\ \mathrm{O} & 2.539810 & 2.963089 & -4.178192 \\ \mathrm{C} & 2.727493 & 2.118500 & 1.557541 \\ \mathrm{O} & -5.677597 & 5.814224 & -0.956146 \\ \mathrm{O} & -6.826202 & 1.323029 & 0.853531 \\ \mathrm{O} & -0.862656 & 5.689651 & -0.772964 \\ \mathrm{O} & 2.750931 & -2.624359 & 0.695114 \\ \mathrm{~W} & -1.221222 & 5.208435 & -2.609985 \\ \mathrm{O} & -4.678940 & -6.520233 & -3.063965 \\ \mathrm{O} & -6.698257 & 2.188705 & -4.126007 \\ \mathrm{O} & 2.878540 & -1.559082 & -4.788895 \\ \mathrm{O} & 0.270676 & -5.666534 & -5.630783 \\ \mathrm{O} & -1.417053 & -1.399994 & -7.461487 \\ \mathrm{O} & -0.688736 & 6.668915 & -3.436310 \\ \mathrm{C} & 3.312973 & 3.523346 & 1.250153 \\ \mathrm{H} & 3.459579 & 1.314484 & 1.333267 \\ \mathrm{H} & 2.381271 & 2.035004 & 2.608780 \\ \mathrm{C} & 4.592270 & 3.712021 & 1.982529 \\ \mathrm{H} & 3.526398 & 3.600967 & 0.162369 \\ \mathrm{H} & 2.568436 & 4.298355 & 1.530411 \\ \mathrm{O} & 5.744995 & 3.564697 & 1.679199 \\ \mathrm{O} & 4.294922 & 4.015091 & 3.446777 \\ \mathrm{C} & 5.181595 & 4.633270 & 4.202271 \\ \mathrm{O} & 6.072021 & 5.413193 & 3.904495 \\ \mathrm{O} & 4.928070 & 4.285858 & 5.521210 \\ \mathrm{C} & 5.744888 & 4.950943 & 6.461166 \\ \mathrm{H} & 5.410446 & 4.598953 & 7.461502 \\ \mathrm{H} & 6.826202 & 4.711502 & 6.318787 \\ \mathrm{H} & 5.637970 & 6.060059 & 6.396957\end{array}$

\subsubsection{Transition State}

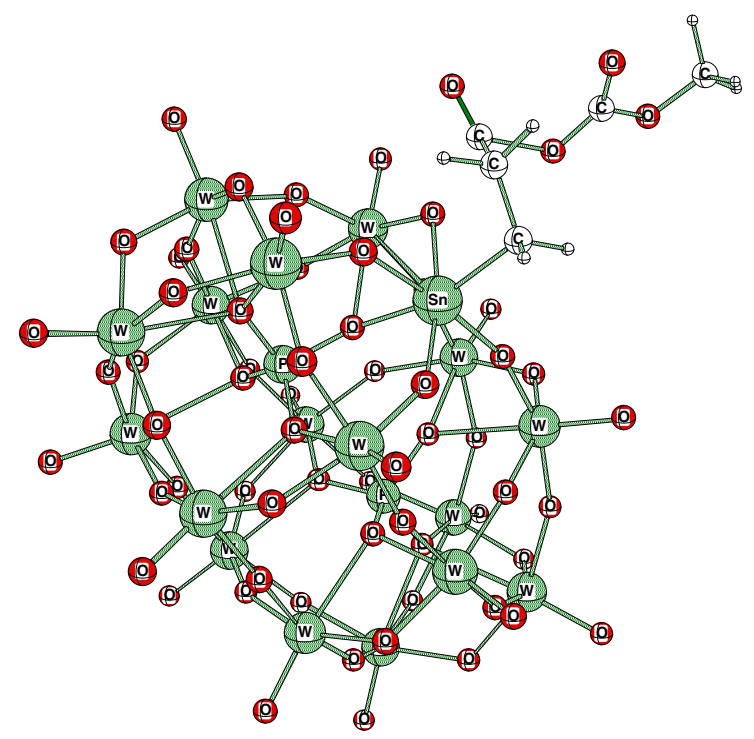

97

Energy $=-6913.0641771860$
O 0.789108
3.324326
0.859848
C 1.700333
4.784821
1.878159
C 1.935333
4.152237
3.227127
C 2.403442
2.704147
3.250961 


\begin{tabular}{|c|c|c|c|}
\hline & 32 & 15 & 2.203049 \\
\hline & 635529 & -1.232666 & 14218 \\
\hline & 1102 & 759 & 91 \\
\hline & -1.771454 & 2358 & 6912 \\
\hline & -5.319275 & 1.077194 & -0.042297 \\
\hline & -3.778763 & & \\
\hline & 1.224350 & -3.744308 & 1.95074 \\
\hline & -0.517593 & 3.113235 & -0.565979 \\
\hline & -1.876816 & -0.927612 & 8563 \\
\hline & & 0.4 & \\
\hline & 2.947845 & -0.921127 & 0.09162 \\
\hline & -0.262711 & -5.613739 & -2.686279 \\
\hline & 297 & 111 & \\
\hline & 409 & & \\
\hline & -2.200150 & 876 & 4958 \\
\hline & 1.305695 & -2.956207 & -4.303741 \\
\hline & 345 & -4.3 & -1.4 \\
\hline & -4.5 & -0.8 & \\
\hline 0 & 0.6 & 907 & -1.5 \\
\hline & -5.6 & 821 & 275 \\
\hline & -1.992950 & 876 & -1.5 \\
\hline & 2.222824 & -2.1 & \\
\hline 0 & -1.92 & -0.9 & \\
\hline & -2.6 & -3 & \\
\hline 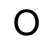 & 0.61 & -0.0 & 3.4 \\
\hline & & 0.1 & -1.5 \\
\hline & $0.1 \varepsilon$ & 085 & \\
\hline & 0.7 & -1 & -0 \\
\hline b & 2.0 & -4.5 & \\
\hline 0 & -5.28 & 0.4 & -1.3 \\
\hline & & & \\
\hline & -2.29 & -2.3 & \\
\hline 0 & -4.46 & -2.205093 & -1.3 \\
\hline 0 & 2.18 & & \\
\hline D & -0.1 & -5 & \\
\hline 0 & -0.4 & $-3 .($ & \\
\hline 0 & -0.8 & & 0.9 \\
\hline 0 & -3.1 & & \\
\hline b & -0.4 & -2 & -4 \\
\hline 0 & -3.0 & -4.3 & -0 . \\
\hline 0 & -1.5 & & -1 \\
\hline D & & & \\
\hline م & -0.5 & & \\
\hline o & 25 & -2.5 & -0.5 \\
\hline b & & & \\
\hline b & -0.8 & & \\
\hline b & -2.9 & & \\
\hline 0 & -1.954666 & -4.6 & 864 \\
\hline$P$ & & & \\
\hline & -0.1 & -2.3 & -1. \\
\hline 0 & -5.2 & & \\
\hline 0 & -3.0 & & 27 \\
\hline $\mathrm{O}$ & & & \\
\hline $\mathrm{O}$ & -2.5 & -6.5 & \\
\hline 0 & 0.59 & $-3.4 t$ & -2.032 \\
\hline 0 & -4.578873 & -2.223740 & 1.314392 \\
\hline ? & -4.17 & & -0.1 \\
\hline ก & & & \\
\hline $\mathrm{O}$ & 2.314255 & -4.522598 & 3.085068 \\
\hline & 3.033569 & 0.248230 & -2.90593 \\
\hline
\end{tabular}

$\begin{array}{rrrr}\mathrm{O} & -1.698151 & 0.353622 & 4.736603 \\ \mathrm{O} & -1.972916 & 2.987122 & 5.957092 \\ \mathrm{O} & 1.775070 & -1.222519 & -3.642975 \\ \mathrm{O} & 4.300003 & -5.236160 & -1.378677 \\ \mathrm{O} & -4.123413 & 1.648865 & 4.573425 \\ \mathrm{O} & -0.396362 & 0.199936 & -3.385849 \\ \mathrm{O} & -4.740021 & -4.557144 & -2.863739 \\ \mathrm{O} & -4.532410 & 2.613754 & -2.909683 \\ \mathrm{O} & -0.251083 & 4.592163 & -1.455551 \\ \mathrm{O} & -6.806793 & 0.895355 & 3.979233 \\ \mathrm{O} & -4.760376 & -3.618393 & 3.859848 \\ \mathrm{O} & -2.956024 & 3.711136 & 3.310745 \\ \mathrm{O} & 4.627243 & -0.745300 & 0.576477 \\ \mathrm{~W} & -3.684525 & 3.575745 & 1.519379 \\ \mathrm{O} & -0.615280 & -7.224258 & -3.306046 \\ \mathrm{O} & -6.966888 & -1.649918 & -0.224106 \\ \mathrm{O} & 2.111008 & 1.537231 & -3.875244 \\ \mathrm{O} & 1.913544 & -2.929474 & -5.957092 \\ \mathrm{O} & -2.262314 & -0.312057 & -5.546936 \\ \mathrm{O} & -4.347092 & 5.190781 & 1.299332 \\ \mathrm{O} & 1.168930 & 5.812241 & 1.596939 \\ \mathrm{O} & 3.188873 & 4.572510 & 1.000870 \\ \mathrm{C} & 4.128540 & 5.403275 & 1.280533 \\ \mathrm{O} & 4.278885 & 6.199341 & 2.215591 \\ \mathrm{O} & 5.135880 & 5.319504 & 0.291168 \\ \mathrm{C} & 6.251495 & 6.133698 & 0.530991 \\ \mathrm{H} & 3.372513 & 2.551239 & 2.733887 \\ \mathrm{H} & 2.458710 & 2.323914 & 4.292816 \\ \mathrm{H} & 0.962875 & 4.239426 & 3.761200 \\ \mathrm{H} & 2.664841 & 4.861359 & 3.695419 \\ \mathrm{H} & 6.751770 & 5.904434 & 1.505936 \\ \mathrm{H} & 6.966873 & 5.939133 & -0.302185 \\ \mathrm{H} & 5.995636 & 7.224258 & 0.551575\end{array}$

\subsection{Cyclization : Dawson $\alpha_{2}$}

\subsubsection{Reagent}

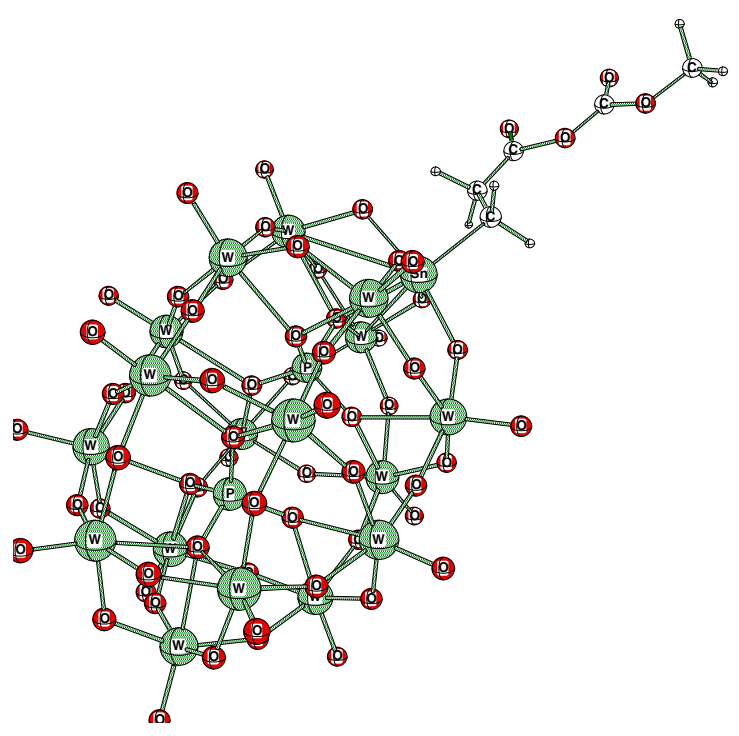

97 


\begin{tabular}{|c|c|c|c|}
\hline \multicolumn{4}{|c|}{ Energy $=-6913.0747373530$} \\
\hline & & & -2.013901 \\
\hline & 1364 & 0147 & \\
\hline & 6330 & -4.434402 & \\
\hline & 0.211472 & -2.776306 & -2.648849 \\
\hline V & 1.723495 & -1.263840 & \\
\hline N & -6.519257 & -1.960388 & 576 \\
\hline 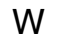 & -0.121246 & 6489 & 468 \\
\hline W & 0.114655 & -3.962433 & 2.3090 \\
\hline N & -1.741943 & -1.264999 & \\
\hline 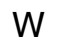 & -3.324402 & 2.370483 & \\
\hline N & -4.918106 & -0.301682 & 3.240204 \\
\hline W & 295 & -6.402603 & \\
\hline N & 672 & & \\
\hline V & -1.3 & -5.475647 & 6169 \\
\hline N & -2.0 & 3.135712 & 4987 \\
\hline N & -2.8 & -4.8 & \\
\hline$N$ & -6.0 & -3.9 & \\
\hline 0 & -2.0 & -0.3 & 248 \\
\hline 0 & -1.1 & & \\
\hline b & 0.3 & -1.0 & -3.6 \\
\hline U & 0.9 & & \\
\hline 0 & -5.8 & -0.7 & \\
\hline 0 & -2.9 & -0 & -1 \\
\hline 0 & -4.2 & -3. & \\
\hline 0 & & 1.51 & -0.2 \\
\hline 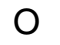 & -3.4 & & \\
\hline 0 & -5.8 & & \\
\hline 0 & -3.1 & -1.6 & \\
\hline 0 & -6.6 & -3.2 & \\
\hline 0 & & & \\
\hline 0 & -5.9 & -5 & \\
\hline 0 & -4.6 & -0.4 & -3.5 \\
\hline 0 & & & \\
\hline 0 & & & \\
\hline 0 & -1.7 & & \\
\hline 0 & -6.4 & -3.4 & -1.0 \\
\hline 0 & & & \\
\hline 0 & -1.7 & & \\
\hline $\mathrm{O}$ & -0.0 & & \\
\hline 0 & -1.2 & & \\
\hline 0 & & & \\
\hline 0 & & & \\
\hline 0 & -4.8 & -4.5 & 48 \\
\hline 0 & & & \\
\hline 0 & & & \\
\hline 0 & & & \\
\hline 0 & -5.0 & -5.5 & \\
\hline 0 & & & \\
\hline 0 & & & \\
\hline $\mathrm{P}$ & -1.56 & -0.1 & -0. \\
\hline$P$ & -3.25 & -2.9 & \\
\hline 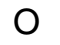 & & & \\
\hline 0 & & & \\
\hline 0 & -6.3 & 392 & -2.5 \\
\hline 0 & -5.493393 & -5.54 & -2.658279 \\
\hline 0 & -3.9 & -4.1 & \\
\hline 0 & & & \\
\hline $\mathrm{O}$ & 1.970947 & 0.287216 & -0.924042 \\
\hline $\mathrm{O}$ & 0.579056 & 2.312714 & 0.500183 \\
\hline
\end{tabular}

$\begin{array}{rrrr}\mathrm{O} & -8.227264 & -1.573380 & 0.130386 \\ \mathrm{O} & 0.971634 & -2.817398 & 1.126358 \\ \mathrm{O} & -3.633987 & 1.983032 & -2.705154 \\ \mathrm{O} & -2.660370 & 4.670792 & -3.175720 \\ \mathrm{O} & -2.550980 & -3.017334 & 4.821442 \\ \mathrm{O} & -7.607300 & -4.062363 & 4.366333 \\ \mathrm{O} & -1.380371 & 2.276276 & -4.205902 \\ \mathrm{O} & -0.446548 & -2.416428 & 3.395584 \\ \mathrm{O} & -0.854477 & -6.955734 & -1.182236 \\ \mathrm{O} & 3.382797 & -1.473572 & 0.663925 \\ \mathrm{O} & 0.854736 & 2.198486 & 3.243942 \\ \mathrm{O} & -4.205658 & 3.801117 & 1.465103 \\ \mathrm{O} & 0.137344 & 0.598343 & -6.030121 \\ \mathrm{O} & -3.636246 & -2.407333 & -5.344559 \\ \mathrm{O} & -0.267624 & 3.635849 & -2.110748 \\ \mathrm{O} & -6.082779 & 0.646515 & 4.149216 \\ \mathrm{Sn} & 1.054749 & 2.063370 & -1.512909 \\ \mathrm{O} & -4.837906 & -8.092987 & 1.537979 \\ \mathrm{O} & 1.003983 & -3.778214 & -3.852036 \\ \mathrm{O} & -0.980881 & -0.906128 & 5.805695 \\ \mathrm{O} & -2.442505 & -5.639313 & 6.030121 \\ \mathrm{O} & 1.517746 & -4.536804 & 3.255569 \\ \mathrm{C} & 2.789093 & 3.436981 & -1.780487 \\ \mathrm{C} & 2.507980 & 4.151428 & -3.100983 \\ \mathrm{H} & 3.719284 & 2.830612 & -1.811203 \\ \mathrm{H} & 2.828201 & 4.132370 & -0.915253 \\ \mathrm{C} & 3.425095 & 5.163361 & -3.691269 \\ \mathrm{H} & 2.320007 & 3.381058 & -3.900330 \\ \mathrm{H} & 1.489883 & 4.628799 & -3.047821 \\ \mathrm{O} & 3.367691 & 5.783844 & -4.716507 \\ \mathrm{O} & 4.619156 & 5.391556 & -2.767181 \\ \mathrm{C} & 5.597137 & 6.236557 & -3.039551 \\ \mathrm{O} & 5.791870 & 6.978027 & -3.988632 \\ \mathrm{O} & 6.491837 & 6.169922 & -1.976898 \\ \mathrm{C} & 7.612549 & 7.018326 & -2.110031 \\ \mathrm{H} & 8.227280 & 6.858704 & -1.197525 \\ \mathrm{H} & 8.216125 & 6.776077 & -3.017212 \\ \mathrm{H} & 7.318878 & 8.093002 & -2.183029\end{array}$

1.5.2 Transition State 


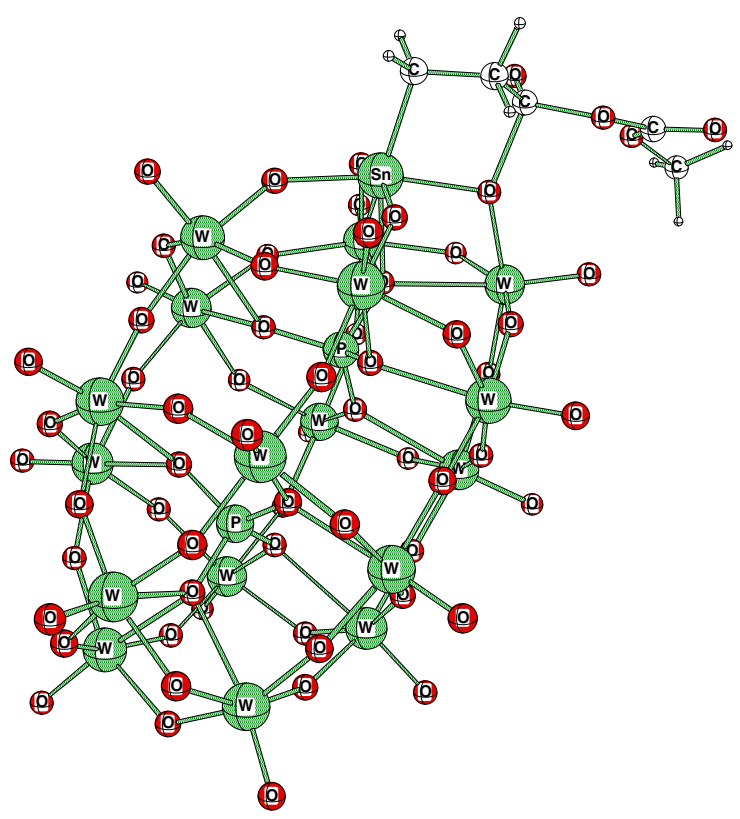

97

Energy $=-6913.0647573030$

$\begin{array}{cccc}\text { Sn } & -2.013203 & 4.803911 & -0.316636 \\ \text { O } & -0.649911 & 5.939854 & 0.986715 \\ \text { C } & -1.442388 & 7.641763 & 1.086115 \\ \text { O } & -2.728488 & 3.646974 & 1.295781 \\ \text { C } & -3.417299 & 6.437882 & 0.082118 \\ \text { C } & -2.869546 & 7.140499 & 1.329642 \\ \text { O } & -0.981112 & 8.065316 & 2.558710 \\ \text { C } & 0.079115 & 8.783359 & 2.855865 \\ \text { W } & 2.927734 & 1.644200 & -2.236190 \\ \text { W } & 3.243912 & 1.883300 & 1.225233 \\ \text { W } & 3.347122 & -1.949814 & 1.543591 \\ \text { W } & 0.126070 & 2.052027 & 3.371446 \\ \text { W } & -3.046049 & 1.908353 & 1.918287 \\ \text { W } & 3.033950 & -2.190155 & -1.907922 \\ \text { W } & -3.380124 & 1.614167 & -1.809570 \\ \text { W } & -2.890994 & -1.937914 & 2.260397 \\ \text { W } & -3.236024 & -2.233443 & -1.497277 \\ \text { W } & -0.486672 & 1.507268 & -3.786987 \\ \text { W } & -0.392749 & -2.339637 & -3.484600 \\ \text { W } & 1.215243 & -5.056792 & 1.985995 \\ \text { W } & 1.117453 & 5.033099 & 1.203927 \\ \text { W } & 0.266825 & -1.788405 & 3.697692 \\ \text { W } & 0.785497 & 4.801448 & -2.305763 \\ \text { W } & -1.947407 & -5.177018 & 0.520482 \\ \text { W } & 0.914181 & -5.303790 & -1.482209 \\ \text { O } & 2.503130 & 3.725114 & 1.040707 \\ \text { O } & -3.272678 & -0.384180 & -1.889146 \\ \text { O } & 0.356326 & 3.854170 & 2.537261 \\ \text { O } & -3.548598 & 1.384846 & 0.116336 \\ \text { O } & 1.465205 & -2.013956 & -3.033084 \\ \text { O } & 1.397421 & 1.492282 & -0.318269 \\ \text { O } & 3.487165 & -0.019300 & 1.239557 \\ \text { O } & 1.377108 & 1.198735 & -3.291084 \\ \text { O } & -2.286049 & -2.637958 & -3.155095 \\ \text { O } & 3.199919 & -0.270819 & -1.941716 \\ \text { O } & -0.844156 & -1.861178 & -1.148122 \\ \text { O } & 2.372251 & -4.021816 & -1.610880\end{array}$

$\begin{array}{llll}\mathrm{O} & -1.794174 & 2.211363 & 3.426162\end{array}$

$\begin{array}{llll}\text { O } & 1.655980 & -5.873100 & 0.268052\end{array}$

$\begin{array}{llll}\text { O } & 3.926211 & 2.005446 & -0.591435\end{array}$

O $0.021444 \quad 0.136505 \quad 3.678188$

$\begin{array}{llll}\text { O } & -0.655726 & -0.460781 & -3.720920\end{array}$

$\begin{array}{llll}\mathrm{O} & -2.402687 & 1.664561 & -3.517982\end{array}$

O $4.051816 \quad-2.284354 \quad-0.238514$

$\begin{array}{llll}\text { O } & 1.528921 & -1.751775 & -0.054920\end{array}$

$\begin{array}{llll}\mathrm{O} & -0.970570 & 1.397681 & -1.411348\end{array}$

$\begin{array}{llll}\mathrm{O} & -1.656708 & -2.067008 & 3.770413\end{array}$

$\begin{array}{llll}\text { O } & -2.463804 & -3.845691 & 1.795749\end{array}$

O $2.005136 \quad-1.527366 \quad 2.881019$

$\begin{array}{llll}\mathrm{O} & -0.606856 & -1.658193 & 1.444901\end{array}$

$\begin{array}{llll}\mathrm{O} & -0.787902 & -6.025206 & -0.850448\end{array}$

O

O

$\begin{array}{llll}\text { O } & -0.556966 & -5.810110 & 1.816067\end{array}$

$\begin{array}{llll}\text { O } & 2.671932 & -3.766968 & 1.679568\end{array}$

$\begin{array}{lllll}\mathrm{O} & -0.147168 & 3.567669 & -0.308815\end{array}$

$\begin{array}{llll}\text { O } & 0.530850 & -3.672559 & 3.166903\end{array}$

$\begin{array}{llll}P & -0.106947 & 1.908935 & -0.207903\end{array}$

$\begin{array}{llll}P & 0.030578 & -2.202227 & 0.114594\end{array}$

$\begin{array}{llll}\text { O } & -0.736542 & 1.556575 & 1.183447\end{array}$

O $4.179065 \quad 1.889795 \quad-3.440599$

$\begin{array}{llll}\text { O } & 4.807513 & -2.162238 & 2.495655\end{array}$

$\begin{array}{llll}\text { O } & 0.052679 & -3.823778 & 0.238194\end{array}$

$\begin{array}{llll}\text { O } & 1.880228 & 1.722801 & 2.581915\end{array}$

$\begin{array}{llll}\text { O } & -3.048959 & 3.448024 & -1.520484\end{array}$

$\begin{array}{llll}\text { O } & 4.301160 & -2.594752 & -3.054434\end{array}$

$\begin{array}{llll}\mathrm{O} & -2.900199 & -0.049019 & 2.326607\end{array}$

$\begin{array}{llll}\text { O } & 2.216330 & 3.448602 & -2.217206\end{array}$

O $1.395464 \quad 5.894020 \quad-3.540983$

$\begin{array}{llll}\text { O } & -2.711403 & -4.062940 & -0.821715\end{array}$

$\begin{array}{llll}\text { O } & 1.462528 & -6.630717 & -2.504248\end{array}$

$\begin{array}{lllll}\mathrm{O} & 1.500220 & 5.577717 & -0.667253\end{array}$

O

O $0.661991 \quad-1.986732 \quad 5.396488$

$\begin{array}{llll}\text { O } & -4.591062 & 2.168629 & 2.712963\end{array}$

$\begin{array}{llll}\mathrm{O} & -5.039779 & 1.737388 & -2.372147\end{array}$

$\begin{array}{llll}\mathrm{O} & -0.414498 & 1.675474 & -5.532074\end{array}$

$\begin{array}{llll}\text { O } & 1.941858 & 6.275167 & 2.134741\end{array}$

$\begin{array}{llll}\text { O } & 4.668822 & 2.342349 & 2.138788\end{array}$

O

O

O $1.950327 \quad-6.202866 \quad 3.103555$

O $0.519105 \quad 2.543629 \quad 5.007663$

$\begin{array}{llll}\mathrm{O} & -4.870640 & -2.671686 & -1.963707\end{array}$

$\begin{array}{llll}\mathrm{O} & -3.156548 & -6.440629 & 0.735830\end{array}$

$\begin{array}{llll}\mathrm{O} & -4.405742 & -2.275736 & 3.081267\end{array}$

H $\quad-4.420698 \quad 5.996029 \quad 0.260984$

H $-3.445239 \quad 7.117403 \quad-0.797128$

H $\quad-3.465473 \quad 8.053999 \quad 1.601154$

H $-2.881135 \quad 6.460445 \quad 2.205399$

$\begin{array}{llll}\text { O } & -1.116862 & 8.359093 & 0.168115\end{array}$

$\begin{array}{llll}\mathrm{O} & 0.287129 & 9.255147 & 3.976284\end{array}$

$\begin{array}{llll}\mathrm{O} & 0.919854 & 9.013817 & 1.803849\end{array}$

C $2.241933 \quad 9.373936 \quad 2.149385$

H $2.764508 \quad 8.491918 \quad 2.582077$

$\begin{array}{llll}\mathrm{H} & 2.739460 & 9.657827 & 1.196057\end{array}$

H $2.263827 \quad 10.228217 \quad 2.871259$ 


\subsection{Intermolecular system}

\subsection{1 $\underline{\text { Conf1 }}$}

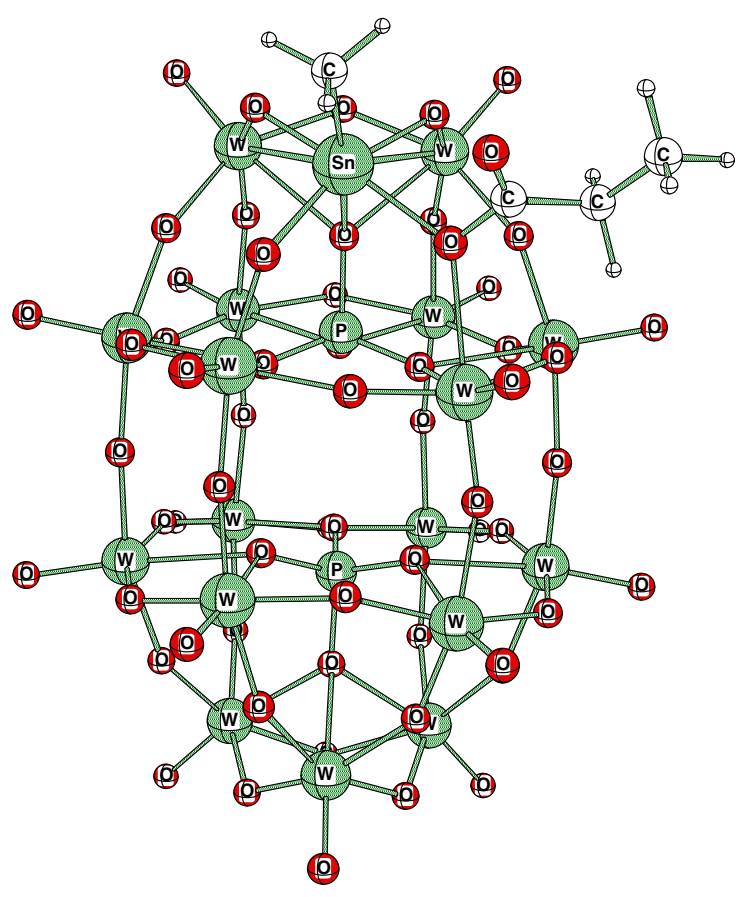

94

\begin{tabular}{lrrr}
\multicolumn{4}{|c}{ Energy $=-6650.3655285890$} \\
W & -2.226624 & 2.613083 & -3.091965 \\
W & 1.257355 & 2.501343 & -3.200562 \\
W & 1.137405 & -1.277298 & -3.959030 \\
W & 3.246948 & 1.899857 & -0.088074 \\
W & 1.644104 & 1.046677 & 2.884201 \\
W & -2.327042 & -1.145401 & -3.839233 \\
W & -2.162308 & 1.466187 & 3.083420 \\
W & 1.530106 & -2.718155 & 2.139771 \\
W & -2.203903 & -2.341187 & 2.332123 \\
W & -3.980972 & 2.012009 & 0.169159 \\
W & -4.057861 & -1.773605 & -0.546997 \\
W & 1.151184 & -4.727661 & -2.401810 \\
W & 1.397400 & 5.214417 & -0.504486 \\
W & 3.146225 & -1.891830 & -0.826202 \\
W & -2.123016 & 5.307419 & -0.413605 \\
W & -0.617874 & -5.350311 & 0.544144 \\
W & -2.346603 & -4.628860 & -2.367905 \\
$O$ & 1.245316 & 4.129852 & -2.140045 \\
$O$ & -2.331604 & -0.481461 & 2.690979 \\
$O$ & 2.637741 & 3.733856 & 0.009033 \\
$O$ & -0.201920 & 1.056122 & 3.249023 \\
$O$ & -3.492371 & -1.141434 & -2.292206 \\
$O$ & -0.398453 & 1.943298 & -1.485245 \\
$O$ & 1.071213 & 0.645935 & -3.759476 \\
$O$ & -3.424545 & 2.018066 & -1.686249 \\
$O$ & -3.852890 & -2.369522 & 1.303253 \\
$O$ & -2.125366 & 0.741989 & -3.660034 \\
$O$ & -1.721039 & -1.630127 & 0.083557 \\
$O$ & -2.277344 & -3.107178 & -3.518921
\end{tabular}

\begin{tabular}{|c|c|c|c|}
\hline & 60 & 325 & 801 \\
\hline & 8325 & -5.288818 & -3.049515 \\
\hline & -0.494125 & 912888 & -3.930862 \\
\hline & 3.304626 & -0.089218 & -0 . \\
\hline & -4.114716 & 0.081421 & 0.03410 \\
\hline & -3.797424 & 1.846603 & 2.126205 \\
\hline & -0.628082 & -1.293381 & 777893 \\
\hline & -0.498596 & -1.231277 & -2.183029 \\
\hline & -1.672607 & 1.558456 & 0.744980 \\
\hline & 3.089981 & -2.602066 & 1.008987 \\
\hline & 0.888672 & -4.331436 & 1. \\
\hline & 2.449265 & -1.303162 & -2.519119 \\
\hline & 0.882721 & -1.745499 & -0.012619 \\
\hline & -1.980927 & -5.741943 & -0.742371 \\
\hline & -3.314606 & 3.864227 & 0.16 \\
\hline & -3.469803 & -3.521835 & -1.218185 \\
\hline & 0.729630 & -5.806931 & -0.813614 \\
\hline & 1.020309 & -3.209549 & -3.607788 \\
\hline & -0.320969 & 3.713989 & 0.410858 \\
\hline & 2.417801 & -3.679688 & -1.434052 \\
\hline & -0.371582 & 2.111191 & 0.066406 \\
\hline & -0.465408 & -1.973328 & -0.798828 \\
\hline & 1.199081 & 5.679214 & 1.33242 \\
\hline & 0.946243 & 1.517548 & 0.716324 \\
\hline & -3.311295 & 3.199371 & -4.332153 \\
\hline & 2.137634 & -1.366852 & -5.392853 \\
\hline & -0.538040 & -3.564484 & -1.117706 \\
\hline & 2.549606 & 1.877991 & -1.875229 \\
\hline & 1.423737 & 592 & 922 \\
\hline & -1.612183 & 3.265778 & 3.096527 \\
\hline & -3.442215 & -1.185318 & -5.187149 \\
\hline & 1.867676 & -0.731476 & 2.559052 \\
\hline & -2.048309 & 4.224075 & -2.069016 \\
\hline 0 & -3.193680 & 6.608932 & -0.902786 \\
\hline & -1.722168 & -4.136002 & 1.519211 \\
\hline & -3.493652 & -5.676498 & 357 \\
\hline & -0.342911 & 5.991180 & -0.931473 \\
\hline 0 & -0.279984 & -2.281281 & 2.640213 \\
\hline & 4.82 & -2.229645 & -1.202209 \\
\hline & 2.349701 & 1.17 & 327 \\
\hline & -2.758713 & 1.368469 & 4.728119 \\
\hline & -5.683685 & 2.404327 & 0.075943 \\
\hline & 2.487503 & $6.4 \varepsilon$ & -1.024185 \\
\hline & 2.281555 & 3.065292 & -4.501907 \\
\hline & -1.786942 & 5.787003 & 1.372604 \\
\hline 0 & -5.776093 & -2.027618 & -0.760330 \\
\hline & -0.3 & 4.762756 & 540 \\
\hline & 2.154633 & -5.856354 & -3.297577 \\
\hline & 4.954254 & 2.214676 & -0.311615 \\
\hline & -2.798248 & -2.979340 & 3.85054 \\
\hline & -0.643631 & -6.815948 & 1.50968 \\
\hline & 2.236969 & -3.321152 & 3.623871 \\
\hline & -0.534317 & 6.106735 & 4.185654 \\
\hline & 0.317657 & 6.815948 & 4.206848 \\
\hline & -0.556824 & 5.510361 & 5.11773 \\
\hline & -1.494720 & 6.635757 & 4.00613 \\
\hline & 2.315308 & 4.101776 & 3.937714 \\
\hline & 2.001495 & 4.750244 & 4.93631 \\
\hline & & 4.068695 & \\
\hline & & & \\
\hline
\end{tabular}



H 3.751053
4.573349
2.454391
H 4.043503
3.021805
H 5.776093
4.723312
3.213287
H 4.455276
5.791794
3.992828
H 4.758591
4.206741
4.618439
5.392853

\subsection{2 $\underline{\text { Conf2 }}$}

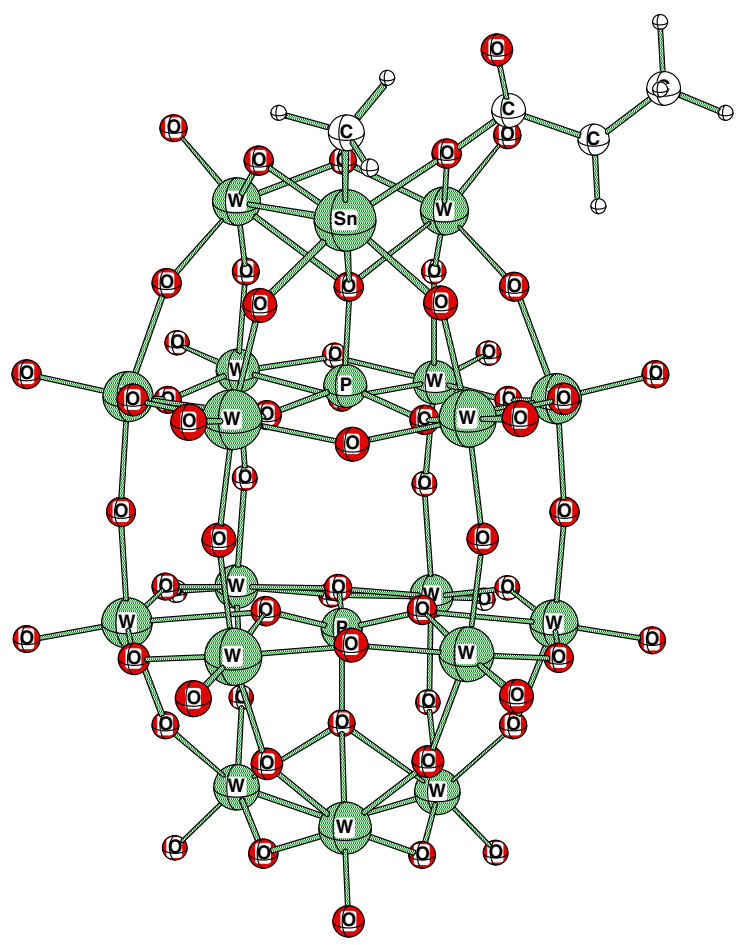

94

\begin{tabular}{lrrr}
\multicolumn{4}{c}{ Energy $=-6650.3699231710$} \\
W & -2.164459 & 0.833282 & 3.349579 \\
O & -1.736267 & 2.659836 & 3.261887 \\
Sn & -0.373703 & 4.053650 & 2.571350 \\
O & 1.151337 & 2.702835 & 3.035461 \\
C & 2.267319 & 5.877655 & 2.033478 \\
C & 3.690460 & 5.437775 & 1.691696 \\
C & 4.762604 & 6.192230 & 2.481232 \\
W & -2.300705 & 1.786758 & -2.899185 \\
W & 1.164139 & 1.595535 & -3.202835 \\
W & 1.115173 & -2.201080 & -3.741943 \\
W & 3.163315 & 1.228424 & 0.026398 \\
W & 1.591248 & 0.863556 & 3.118744 \\
W & -2.343292 & -2.023865 & -3.505768 \\
W & 1.589630 & -2.941437 & 2.468597 \\
W & -2.183350 & -2.958633 & 2.693649 \\
W & -4.019714 & 1.263245 & 0.415161 \\
W & -4.064331 & -2.539917 & -0.194656 \\
W & 1.199875 & -5.542709 & -1.980789 \\
W & 1.288696 & 4.441177 & -0.757935 \\
W & 3.149033 & -2.573456 & -0.611786 \\
W & -2.225235 & 4.559448 & -0.302170 \\
W & -0.394241 & -5.874573 & 1.088623 \\
W & -2.272079 & -5.441010 & -1.836166 \\
O & 1.142807 & 3.494888 & -2.313248 \\
O & -2.395233 & -1.091949 & 2.947052 \\
O & 2.459747 & 3.120041 & -0.010468
\end{tabular}

\begin{tabular}{|c|c|c|c|}
\hline & 562 & & \\
\hline & 1322 & 77829 & -1.964981 \\
\hline & -0.462891 & 1.280258 & -1.3751 \\
\hline & 0.992844 & -0.201965 & -3.66 \\
\hline & -3.450470 & 1.252640 & -1.451157 \\
\hline & -3.838104 & -3.058533 & 1.660233 \\
\hline & -2.186600 & -0.112137 & 0.7000 \\
\hline & -1.709473 & -2.314163 & 0.370789 \\
\hline & -2.237793 & -3.965347 & -3.082031 \\
\hline & 3.168442 & 1.197311 & 1.945709 \\
\hline & -0.560303 & -6.104965 & -2.586380 \\
\hline & -0.604538 & 2.071259 & -3.835602 \\
\hline & 3.295441 & -0.671158 & -0.129639 \\
\hline & -4.141678 & -0.659576 & 0.301086 \\
\hline & -3.817429 & 1.173767 & 2.332092 \\
\hline & -0.662918 & -2.195755 & -4.505295 \\
\hline & -0.515625 & -2.000534 & -1.937180 \\
\hline & -1.676926 & 0.942184 & 0.915436 \\
\hline & 3.132202 & -3.060806 & 1.260727 \\
\hline & 0.973389 & -4.731995 & 1.790451 \\
\hline & 2.420898 & -2.008377 & -2.321152 \\
\hline & 0.898941 & -2.3 & 0.226837 \\
\hline & -1.777390 & -6.423584 & -0.213974 \\
\hline & -3.378922 & 3.156494 & 0.334686 \\
\hline & -3.444107 & -4.312622 & -0.784760 \\
\hline & 0.902145 & -6.465744 & -0.333054 \\
\hline & 1.060669 & -4.052475 & -3.294281 \\
\hline & -0.329041 & 9167 & 5591 \\
\hline & 2.471664 & -4.338379 & -1.124435 \\
\hline & -0.395508 & 1.453751 & 0.180573 \\
\hline & -0.449966 & -2.653122 & -0.506439 \\
\hline & 1.288849 & 5.223083 & 1.402298 \\
\hline & 0.923065 & 0.864090 & 0.785065 \\
\hline & -3.429779 & 2.312546 & -4.127487 \\
\hline & 2.09 & -2.3 & -5.1904 \\
\hline & -0.462814 & -4.262650 & -0.735992 \\
\hline & 2.409729 & 1.240829 & -1.796890 \\
\hline & -3.472748 & -2.147736 & -4.837677 \\
\hline & 1.82 & -1.077454 & 35 \\
\hline & -2.126984 & 3.471756 & -1.944031 \\
\hline & -3.316528 & 5.846527 & -0.781143 \\
\hline & -1.660873 & -4.747345 & 1.959473 \\
\hline & -3.389618 & -6.5 & -2.582443 \\
\hline & -0.434280 & 5.294678 & -0.863174 \\
\hline & -0.275925 & -2.769226 & 2.963181 \\
\hline & 4.835007 & -2.859116 & -0.985123 \\
\hline & 2.367706 & 0.8 & 38980 \\
\hline & -2.772675 & 0.781723 & 4.991135 \\
\hline & -5.723343 & 1.645737 & 0.305786 \\
\hline & 2.369308 & 5.76 & -1.198135 \\
\hline & 2.167938 & 276 & -4.523773 \\
\hline & -1.793427 & 5.115051 & 1.462799 \\
\hline & -5.780167 & -2.826309 & -0.389648 \\
\hline & 2.241852 & -6.672485 & -2.827377 \\
\hline & 4.837753 & 1.655533 & -0.260422 \\
\hline & -2.745529 & -3.539169 & 4.246124 \\
\hline & -0.330582 & -7.287964 & 2.128937 \\
\hline & 2.325165 & -3.529816 & 3.943878 \\
\hline & -0.354904 & 5.3 & 4.343491 \\
\hline & 0.3017 & 6.180862 & 4.154510 \\
\hline
\end{tabular}




$\begin{array}{rrrr}\mathrm{H} & 0.012436 & 4.693497 & 5.190445 \\ \mathrm{H} & -1.409943 & 5.613815 & 4.509781 \\ \mathrm{O} & 2.060287 & 6.764999 & 2.865784 \\ \mathrm{H} & 3.834137 & 5.554626 & 0.595398 \\ \mathrm{H} & 3.741135 & 4.340149 & 1.868256 \\ \mathrm{H} & 5.780167 & 5.826431 & 2.208557 \\ \mathrm{H} & 4.716400 & 7.287964 & 2.282822 \\ \mathrm{H} & 4.624344 & 6.056519 & 3.576935\end{array}$

\subsection{3 $\underline{\text { Conf3 }}$}

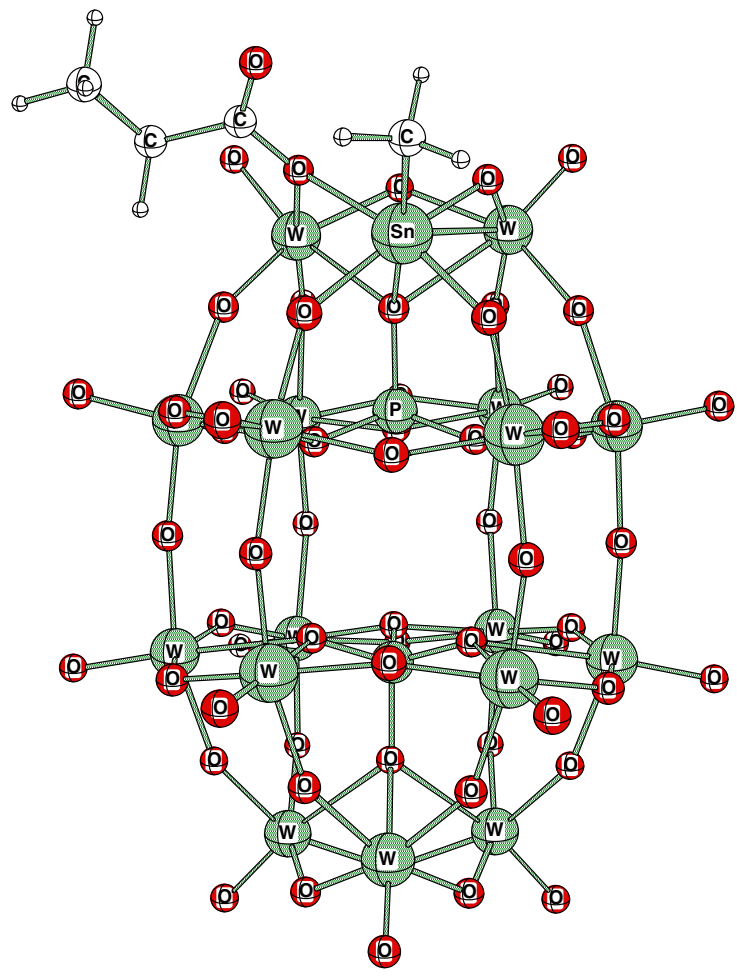

94

\begin{tabular}{lccc}
\multicolumn{4}{c}{ Energy $=-6650.3696430840$} \\
Sn & 0.426788 & 3.950043 & 2.325470 \\
O & -1.239075 & 5.040619 & 1.123062 \\
W & -1.313309 & 4.127274 & -0.986557 \\
O & 0.420380 & 4.936722 & -1.190765 \\
W & 2.211899 & 4.200043 & -0.625580 \\
O & 1.837128 & 4.891861 & 1.105148 \\
W & -1.587143 & 0.841095 & 3.141190 \\
O & -1.102264 & 2.657227 & 2.927322 \\
O & 1.782455 & 2.569199 & 3.058640 \\
C & -2.161148 & 5.770889 & 1.757996 \\
C & -3.612640 & 5.473129 & 1.385117 \\
C & -4.620895 & 6.312042 & 2.173447 \\
W & -1.312943 & 1.112259 & -3.224472 \\
W & 2.160461 & 1.255890 & -3.024445 \\
W & 2.105957 & -2.589050 & -3.362274 \\
W & 3.958328 & 0.921478 & 0.274216 \\
W & 2.169571 & 0.744949 & 3.275986 \\
W & -1.359299 & -2.713379 & -3.490234 \\
W & 2.108734 & -3.083099 & 2.891632 \\
W & -1.667343 & -3.001526 & 2.767822 \\
W & -3.231598 & 1.021973 & 0.071899 \\
W & -3.308136 & -2.816223 & -0.286148
\end{tabular}

\begin{tabular}{|c|c|c|c|}
\hline & 2.015854 & -5.881866 & -1.454346 \\
\hline & 3.911804 & -2.914627 & -0.072021 \\
\hline & 0.218185 & -6.067352 & \\
\hline & -1.459244 & -5.917709 & -1.49200 \\
\hline & 2.042999 & 3.006409 & -2.188721 \\
\hline & -1.861603 & -1.119278 & 2.883026 \\
\hline & 3.352737 & 2.821640 & 0.073761 \\
\hline & 0.265045 & 0.416733 & 3.52671 \\
\hline & -2.619095 & -2.391113 & -2.052155 \\
\hline & 0.352219 & 0.894623 & -1.424194 \\
\hline & 1.992722 & -0.674454 & -3.394928 \\
\hline & -2.528625 & 0.887527 & -1.764465 \\
\hline & -3.247238 & -3.170914 & 1.614609 \\
\hline & -1.194702 & -0.716461 & -3.558060 \\
\hline & -1.030792 & -2.547791 & 0.469498 \\
\hline & -1.328323 & -4.529633 & -2.913528 \\
\hline & 3.805588 & 0.975952 & 2.192902 \\
\hline & 0.271133 & -6.561279 & -2.10467 \\
\hline & 0.444626 & 1.504593 & -3.936890 \\
\hline & 4.036728 & -1.003922 & 0.288239 \\
\hline & -3.403961 & -0.881302 & 0.060547 \\
\hline & -3.184799 & 1.126617 & 1.99043 \\
\hline & 0.395279 & -2.799835 & -4.300812 \\
\hline & 0.325577 & -2.421600 & -1.750519 \\
\hline & -0.982422 & 0.666580 & 0.796066 \\
\hline & 3.730042 & -3.291931 & 1.821289 \\
\hline & 1.530334 & -4.907791 & 2.301926 \\
\hline & 3.318863 & -2.464630 & -1.861404 \\
\hline & 1.579788 & -2.595367 & 0.540237 \\
\hline & -1.131729 & -6.728409 & 0.208923 \\
\hline & -2.501144 & 2.895309 & -0.118637 \\
\hline & -2.680664 & -4.627487 & -0.690048 \\
\hline & 1.550552 & -6.734726 & 0.250290 \\
\hline & 1.975739 & -4.496826 & -2.796616 \\
\hline & 0.306549 & 2.8 & 5004 \\
\hline & 3.240845 & 9076 & -0.514236 \\
\hline & 0.332077 & 1.180298 & 0.117126 \\
\hline & 0.289215 & -2.968369 & -0.274704 \\
\hline & 1.621017 & 0.6 & \\
\hline & -2.339935 & 1.597183 & -4.555786 \\
\hline & 3.191849 & -2.835236 & -4.713440 \\
\hline & 0.262817 & -4.589905 & -0.388504 \\
\hline & 3.334991 & & -1.5 \\
\hline & -2.380966 & -2.895615 & -4.901291 \\
\hline & 2.363220 & -1.208359 & 3.003372 \\
\hline & -1.228638 & 3.070206 & -2.472397 \\
\hline & -2.369904 & 5.44 & -1.4 \\
\hline & -1.106689 & -4.848038 & 2.204468 \\
\hline & -2.547058 & -7.081772 & -2.227280 \\
\hline & 0.214203 & -2.834183 & 3.199707 \\
\hline & 5.615601 & -3.246552 & -0.297485 \\
\hline & 2.820328 & 0.800461 & 4.901276 \\
\hline & -2.323227 & 0.943466 & 4.727570 \\
\hline & -4.905365 & 1.461136 & -0.200394 \\
\hline & 3.315933 & 5.426498 & -1.22139 \\
\hline & 3.265762 & 1.668747 & -4.316284 \\
\hline & -5.009445 & -3.090790 & -0.591660 \\
\hline & 3.089203 & -7.085999 & -2.148361 \\
\hline & 5.664795 & 1.260391 & $0.0917 \mathrm{~s}$ \\
\hline & -2.3735 & -3.465042 & 4.300 \\
\hline
\end{tabular}




$\begin{array}{rrrr}\mathrm{O} & 0.157349 & -7.402084 & 2.688293 \\ \mathrm{O} & 2.707413 & -3.563080 & 4.465042 \\ \mathrm{C} & 0.491821 & 5.300156 & 4.027161 \\ \mathrm{H} & 0.713516 & 6.322937 & 3.663727 \\ \mathrm{H} & 1.301682 & 4.911270 & 4.680557 \\ \mathrm{H} & -0.485214 & 5.281601 & 4.548355 \\ \mathrm{O} & -1.879852 & 6.615250 & 2.610748 \\ \mathrm{H} & -3.769058 & 4.382385 & 1.541214 \\ \mathrm{H} & -3.722595 & 5.620361 & 0.287811 \\ \mathrm{H} & -5.664795 & 6.050613 & 1.880249 \\ \mathrm{H} & -4.513504 & 6.144394 & 3.268387 \\ \mathrm{H} & -4.467514 & 7.402069 & 1.997391\end{array}$

\subsection{4 $\underline{\text { Conf4 }}$}

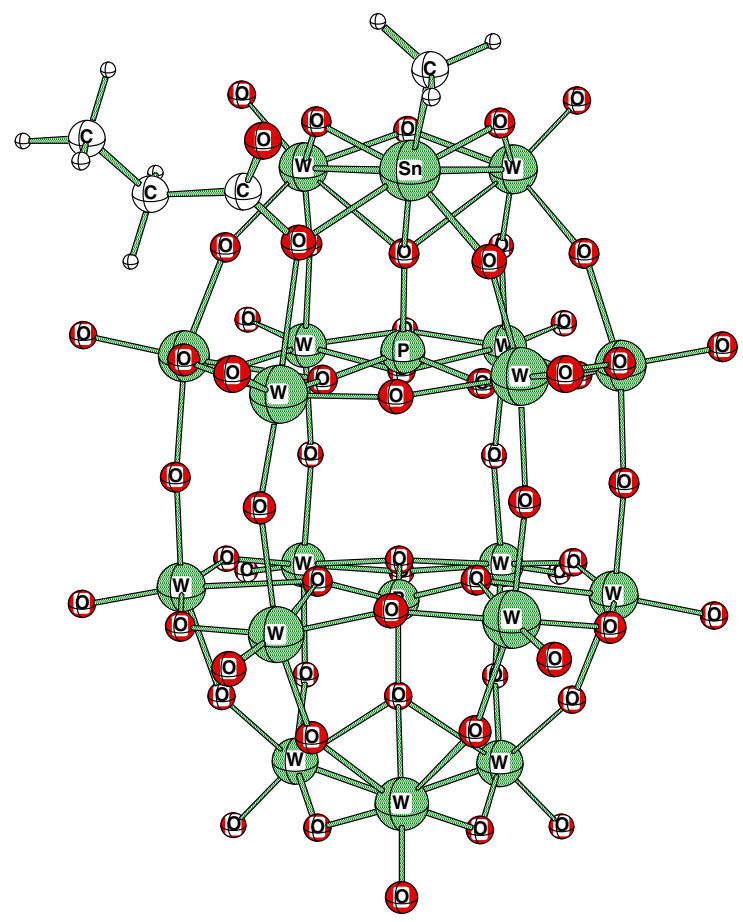

94

\begin{tabular}{cccc}
\multicolumn{4}{c}{ Energy $=-6650.3655383060$} \\
W & 2.166382 & 4.963562 & -0.817520 \\
$O$ & 0.402664 & 5.673218 & -1.344131 \\
W & -1.357559 & 4.999100 & -0.821045 \\
$O$ & -1.092651 & 5.593414 & 0.970032 \\
Sn & 0.412140 & 4.706696 & 2.157593 \\
O & 1.897552 & 5.587082 & 0.935699 \\
O & 1.671188 & 3.210968 & 2.851837 \\
$C$ & -2.203766 & 4.263275 & 3.704895 \\
O & -1.845047 & 4.972214 & 4.645508 \\
$C$ & -3.655624 & 4.255875 & 3.239014 \\
C & -4.588943 & 5.052979 & 4.153687 \\
W & -1.379044 & 2.091934 & -3.308075 \\
W & 2.108414 & 2.068314 & -3.280411 \\
W & 2.046555 & -1.736481 & -3.741135 \\
W & 3.916443 & 1.653702 & -0.019836 \\
W & 2.150543 & 1.395004 & 2.966003 \\
W & -1.422150 & -1.734344 & -3.777878 \\
W & -1.673172 & 1.119202 & 2.880386 \\
W & 2.029816 & -2.456314 & 2.505005 \\
W & -1.719254 & -2.692627 & 2.421890
\end{tabular}

\begin{tabular}{|c|c|c|c|}
\hline & 314728 & 7714 & \\
\hline & 527 & 8391 & 65842 \\
\hline & 1.974411 & -5.093109 & -2.00448 \\
\hline & 3.833893 & -2.175018 & -0.44 \\
\hline & 0.284378 & -5.523758 & 0.9867 \\
\hline & -1.523895 & -5.056976 & -1.964325 \\
\hline & 2.015350 & 8667 & -2.3795 \\
\hline & -1.970337 & -0.668961 & 2.692535 \\
\hline & 3.320190 & 3.521271 & -0.14752 \\
\hline & 0.180145 & 1.079239 & 3.204758 \\
\hline & -2.69 & -1.601028 & -2 \\
\hline & 0.295868 & 1.598022 & -1.58926 \\
\hline & 1.922012 & 0.166779 & -3.702347 \\
\hline & -2.660400 & 1.620605 & -1.912003 \\
\hline & -3.301270 & -2.602493 & \\
\hline & -1.277283 & 0.192871 & -3.728271 \\
\hline & -1.087845 & -1.915146 & 0.187668 \\
\hline & -1.369095 & -3.639008 & 2242 \\
\hline & 3.774826 & 1.637115 & 1.946945 \\
\hline & 0.244324 & -5.735916 & -2.601425 \\
\hline & 0.36 & 2.375153 & 3378 \\
\hline & 3.974625 & -0.289230 & -0. \\
\hline & -3.449905 & -0.187790 & -0.112030 \\
\hline & -3.21 & & \\
\hline & 0.32 & -1.885345 & 9959 \\
\hline & 0.258163 & -1.622086 & -2.0 \\
\hline & -1.010941 & 1.392075 & 0.6 \\
\hline & 543 & 6282 & 6686 \\
\hline & 1.4 & 531 & 087 \\
\hline & 3.249207 & -1.660172 & -2.2 \\
\hline & 1.51 & -1.8 & \\
\hline & -1.1 & -6.0 & -0 \\
\hline & -2.635727 & 3.613754 & 3843 \\
\hline 0 & -2.729462 & -3.889038 & -1.050415 \\
\hline & $1.6 \mathrm{C}$ & -6.0 & -0.293625 \\
\hline & 1.93 & -3.6 & 313 \\
\hline & 0.330917 & 3.507233 & 0.1 \\
\hline & 3.163895 & -3.948563 & -0. \\
\hline & 0.3 & & \\
\hline & 0.2 & -2.2 & 61 \\
\hline & 1.607437 & 1.331161 & 7161 \\
\hline & -2.412247 & 268 & -4.6 \\
\hline & & -1.9 & -5 \\
\hline & 0.23 & -3.8 & $-0 . \varepsilon$ \\
\hline & 3.316467 & 1.535599 & -1.858536 \\
\hline & -1.35 & 91 & 382 \\
\hline & -2.4 & -1.8 & -5 \\
\hline & 2.23 & -0.5 & 2.7 \\
\hline & -1.280045 & 3.793137 & -2.37516 \\
\hline & -2.4 & 6.27 & -1.412415 \\
\hline & -1.15 & -4.381454 & \\
\hline & -2.590851 & -6.209183 & -2.749924 \\
\hline & 0.117630 & -2.294647 & 2.848923 \\
\hline & 5.534988 & -2.514511 & -0.6 \\
\hline & 2.783234 & 1.398331 & $4.60025 c$ \\
\hline & -2.336792 & 1.394547 & 4.48428 \\
\hline & -5.013290 & 2.163986 & -0.328720 \\
\hline & 3.271957 & 6.181366 & -1.42854 \\
\hline & & 2.51 & -4.583817 \\
\hline & & & \\
\hline
\end{tabular}

W $\quad-3.314728 \quad 1.797714 \quad-0.117020$

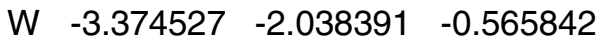

W $\quad 1.974411 \quad-5.093109 \quad-2.004486$

W $3.833893 \quad-2.175018 \quad-0.446823$

W $\quad 0.284378 \quad-5.523758 \quad 0.986710$

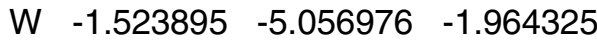

$\begin{array}{lllll}\text { O } & 2.015350 & 3.758667 & -2.379501\end{array}$

$\begin{array}{llll}\mathrm{O} & -1.970337 & -0.668961 & 2.692535\end{array}$

$\begin{array}{llll}\mathrm{O} & 3.320190 & 3.521271 & -0.147522\end{array}$

$\begin{array}{llll}\mathrm{O} & 0.180145 & 1.079239 & 3.204758\end{array}$

$\begin{array}{llll}\text { O } & -2.697800 & -1.601028 & -2.312866\end{array}$

$\begin{array}{lllll}\mathrm{O} & 0.295868 & 1.598022 & -1.589264\end{array}$

\begin{tabular}{llll}
0 & 1.922012 & 0.166779 & -3.702347 \\
\hline & -2.660400 & 1.620605 & -1.912003
\end{tabular}

$\begin{array}{llll}O & -3.301270 & -2.602493 & 1.317749\end{array}$

$\begin{array}{llll}\mathrm{O} & -1.277283 & 0.192871 & -3.728271\end{array}$

$\begin{array}{llll}0 & -1.087845 & -1.915146 & 0.187668\end{array}$

$\begin{array}{llll}0 & -1.369095 & -3.639008 & -3.282242\end{array}$

$\begin{array}{llll}\text { O } & 3.774826 & 1.637115 & 1.946945\end{array}$

$\begin{array}{llll}O & 0.244324 & -5.735916 & -2.601425\end{array}$

$\begin{array}{llll}O & 0.368790 & 2.375153 & -4.103378\end{array}$

$\begin{array}{llll}\text { O } & 3.974625 & -0.289230 & -0.010818\end{array}$

$\begin{array}{llll}0 & -3.449905 & -0.187790 & -0.112030\end{array}$

$\begin{array}{llll}\mathrm{O} & -3.218338 & 1.695679 & 1.832184\end{array}$

$\begin{array}{llll}O & 0.321320 & -1.885345 & -4.629959\end{array}$

$\begin{array}{llll}\text { O } & 0.258163 & -1.622086 & -2.044983\end{array}$

$\begin{array}{lrrr}O & -1.010941 & 1.392075 & 0.665741\end{array}$

$\begin{array}{llll}\text { O } & 3.650543 & -2.626282 & 1.446686\end{array}$

$\begin{array}{llll}\text { O } & 1.458969 & -4.284531 & 1.842087\end{array}$

$\begin{array}{llll}\text { O } & 3.249207 & -1.660172 & -2.223907\end{array}$

$\begin{array}{llll}\text { O } & 1.519653 & -1.896271 & 0.219193\end{array}$

$\begin{array}{llll}\mathrm{O} & -1.110321 & -6.027176 & -0.304245\end{array}$

$\begin{array}{llll}O & -2.635727 & 3.613754 & -0.173843\end{array}$

$\begin{array}{rrrr}\mathrm{O} & -2.729462 & -3.889038 & -1.050415 \\ \mathrm{O} & 1.601639 & -6.065994 & -0.293625\end{array}$

$\begin{array}{llll}\text { O } & 1.931778 & -3.663559 & -3.270813\end{array}$

$\begin{array}{llll}O & 0.330917 & 3.507233 & 0.169189\end{array}$

$\begin{array}{llll}\text { O } & 3.163895 & -3.948563 & -0.969879\end{array}$

$\begin{array}{lllll}\mathrm{P} & 0.312500 & 1.881805 & -0.054428\end{array}$

$\begin{array}{lllll}P & 0.230896 & -2.254944 & -0.607361\end{array}$

$\begin{array}{lllll}\mathrm{O} & 1.607437 & 1.331161 & 0.637161\end{array}$

$\begin{array}{llll}O & -2.412247 & 2.596268 & -4.627045\end{array}$

$\begin{array}{llll}\text { O } & 3.126328 & -1.921448 & -5.105484\end{array}$

$\begin{array}{lllll}\text { O } & 0.235611 & -3.867401 & -0.805542\end{array}$

$\begin{array}{llll}\text { O } & 3.316467 & 1.535599 & -1.858536\end{array}$

$\begin{array}{llll}\text { O } & -1.353302 & 3.515091 & 2.978882\end{array}$

$\begin{array}{llll}O & -2.459564 & -1.892914 & -5.179153\end{array}$

$\begin{array}{llll}O & 2.236069 & -0.580780 & 2.718933\end{array}$

$\begin{array}{lllll}O & -1.280045 & 3.793137 & -2.375168\end{array}$

$\begin{array}{llll}O & -2.410629 & 6.271851 & -1.412415\end{array}$

$\begin{array}{llll}O & -1.158859 & -4.381454 & 1.813187\end{array}$

$\begin{array}{llll}\mathrm{O} & -2.590851 & -6.209183 & -2.749924\end{array}$

$\begin{array}{llll}O & 0.117630 & -2.294647 & 2.848923\end{array}$

$\begin{array}{llll}\text { O } & 5.534988 & -2.514511 & -0.677048\end{array}$

$\begin{array}{llll}\mathrm{O} & 2.783234 & 1.398331 & 4.600250\end{array}$

$\begin{array}{llll}\text { O } & -2.336792 & 1.394547 & 4.484283\end{array}$

$\begin{array}{llll}O & -5.013290 & 2.163986 & -0.328720\end{array}$

$\begin{array}{llll}\text { O } & 3.184372 & 2.516953 & -4.583817\end{array}$

$\begin{array}{llll}\text { O } & -5.071335 & -2.334137 & -0.878876\end{array}$ 
$\begin{array}{llll}\text { O } & 3.060333 & -6.244904 & -2.761978\end{array}$

$\begin{array}{lllll}O & 5.630386 & 1.968109 & -0.178558\end{array}$

$\begin{array}{llll}\text { O } & -2.412109 & -3.152588 & 3.962570\end{array}$

$\begin{array}{llll}0 & 0.277222 & -6.911942 & 2.060959\end{array}$

$\begin{array}{llll}\text { O } & 2.636551 & -3.000900 & 4.054947\end{array}$

$\begin{array}{llll}\text { C } & 0.725784 & 6.160080 & 3.744812\end{array}$

H $\quad 1.708710 \quad 6.624390 \quad 3.515869$

$\mathrm{H} \quad 0.731583 \quad 5.634460 \quad 4.718521$

$\begin{array}{llll}\mathrm{H} & -0.088959 & 6.911926 & 3.721252\end{array}$

$\begin{array}{llll}\mathrm{H} & -3.992645 & 3.206787 & 3.105301\end{array}$

$\begin{array}{llll}H & -3.643280 & 4.667496 & 2.204193\end{array}$

H $-5.630386 \quad 5.049820 \quad 3.752563$

H $\quad-4.609039 \quad 4.618362 \quad 5.179138$

$\begin{array}{llll}H & -4.251877 & 6.109390 & 4.255295\end{array}$

\subsubsection{OxoConf}

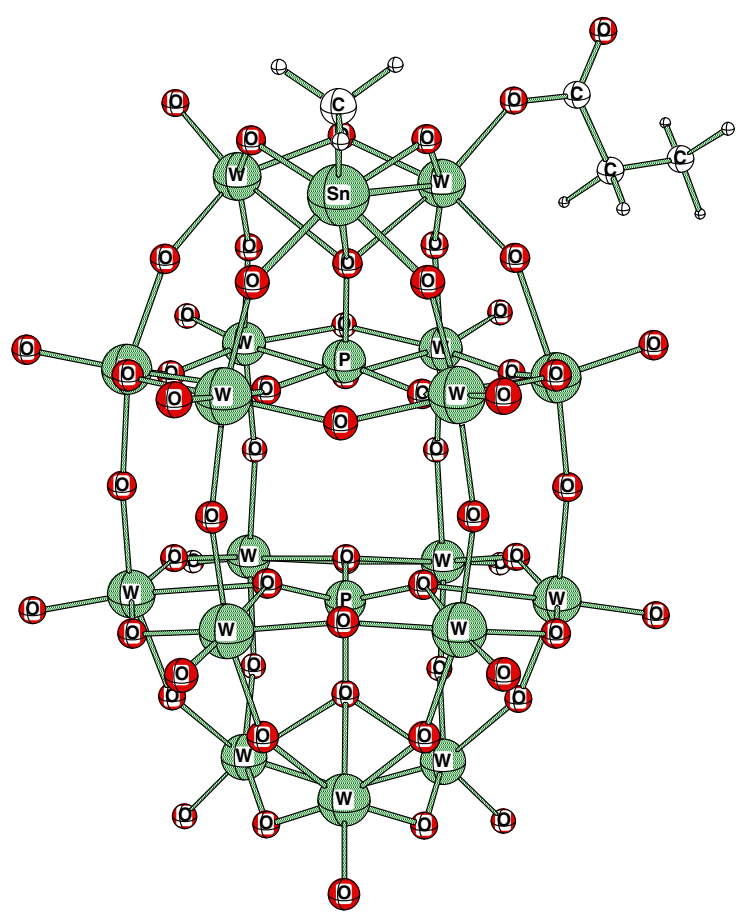

94

Energy $=-6650.3539882470$

$\begin{array}{lrrr}\mathrm{O} & -0.716888 & 5.360901 & -0.939240 \\ \text { W } & -2.489365 & 4.642517 & -0.475525 \\ \mathrm{O} & -2.191254 & 5.101242 & 1.340561 \\ \text { Sn } & -0.734634 & 4.115021 & 2.509048 \\ \mathrm{O} & 0.714401 & 5.130646 & 1.322281 \\ \text { W } & 0.986115 & 4.510452 & -0.436401 \\ \text { W } & -2.471085 & 1.872009 & -3.099396 \\ \text { W } & 0.999176 & 1.788651 & -3.113205 \\ \text { W } & 1.065170 & -1.995346 & -3.778885 \\ \text { W } & 2.900238 & 1.221527 & 0.137512 \\ \text { W } & 1.179138 & 0.874649 & 3.149918 \\ \text { W } & -2.409164 & -1.920135 & -3.762100 \\ \text { W } & -2.569962 & 0.850433 & 3.143250 \\ \text { W } & 1.232056 & -2.923096 & 2.411957 \\ \text { W } & -2.549927 & -2.938217 & 2.424973 \\ \text { W } & -4.314880 & 1.348129 & 0.134583 \\ \text { W } & -4.292404 & -2.441467 & -0.534149 \\ \text { W } & 1.035873 & -5.433075 & -2.161423\end{array}$

\begin{tabular}{|c|c|c|c|}
\hline & & & \\
\hline & -0.665054 & -5.822708 & 0.855209 \\
\hline & -2.436844 & -5.339676 & 126877 \\
\hline & 0.882324 & 3.552307 & -2.06762 \\
\hline & -2.784515 & -1.080307 & 2.705170 \\
\hline & 2.203354 & 3.217056 & 0.141266 \\
\hline & -0.685135 & 0.431305 & 3.421829 \\
\hline & -3.649582 & -1.878784 & -2.274994 \\
\hline & -0.671417 & 1.291443 & -1.499634 \\
\hline & 0.941635 & -0.009979 & -3.674484 \\
\hline & -3.679352 & 1.303436 & -1.7112 \\
\hline & -4.145416 & -3.036118 & 1.30612 \\
\hline & -2.291656 & 0.002304 & -3.638077 \\
\hline & -1.956543 & -2.247955 & 0.153030 \\
\hline & -2.310135 & -3.849274 & -3.366913 \\
\hline & 2.797089 & 1.208801 & 2.060577 \\
\hline & -0.675323 & -5.979019 & -2.842941 \\
\hline & -0.713882 & 2.218719 & -3.905228 \\
\hline & 3.106262 & -0.630814 & -0.025787 \\
\hline & -4.403122 & -0.601807 & 0.000931 \\
\hline & -4.171921 & 1.204880 & 2.056046 \\
\hline & -0.665070 & -2.018539 & -4.652893 \\
\hline & -0.656219 & -1.882217 & $-2.0823 C$ \\
\hline & -1.965210 & 0.986145 & 0.742538 \\
\hline & 2.834473 & -3.030930 & 1.280884 \\
\hline & 0.660400 & -4.705414 & 1.654495 \\
\hline & 2.304916 & -1.869019 & $-2.2865 \mathrm{C}$ \\
\hline & 0.658615 & -2.260437 & 0.138687 \\
\hline & -1.988602 & -6.359436 & -0.530319 \\
\hline & -3.687225 & 3.199905 & 0.097961 \\
\hline & -3.638168 & -4.229446 & -1.134918 \\
\hline & 0.691269 & -6.377686 & -0.531586 \\
\hline & 0.995544 & -3.878204 & -3.384933 \\
\hline & -0.608047 & 3.122818 & 0.330093 \\
\hline & 908 & 5152 & -1.179794 \\
\hline & -0.659058 & 1.480118 & 0.054886 \\
\hline & -0.657074 & -2.574142 & -0.668198 \\
\hline & 0.643097 & 0.922119 & 0.728928 \\
\hline & -3.5 & 7206 & -4.3 \\
\hline & 2.121506 & -2.070526 & -5.17 \\
\hline & -0.662613 & -4.177383 & -0.936630 \\
\hline & 2.206192 & 1.321091 & -1.673676 \\
\hline & 0.708069 & 2.693298 & 3.067947 \\
\hline & -2.113846 & 2.676727 & 3.107239 \\
\hline & -3.465683 & -2.034988 & -5.152908 \\
\hline & 1.452026 & -1.070755 & 2.717789 \\
\hline & -2.356247 & 3.530685 & -2.097977 \\
\hline & -3.564133 & 5.929550 & $-0.98870 \varepsilon$ \\
\hline & -1.979706 & -4.720993 & 1.67819 \\
\hline & -3.517380 & -6.452179 & -2.950546 \\
\hline & -0.657776 & -2.768921 & 2.803894 \\
\hline & 4.658478 & -2.812149 & -0.875641 \\
\hline & 1.863831 & 0.831207 & 4.761597 \\
\hline & -3.261749 & 0.776993 & 4.750687 \\
\hline & -6.021774 & 1.694489 & -0.032288 \\
\hline & 2.231613 & 5.964310 & -1.016830 \\
\hline & 2.084763 & 2.476883 & -4.308823 \\
\hline & -5.991470 & -2.747299 & -0.819702 \\
\hline & 2.113602 & -6.541183 & -2.99426 \\
\hline & 4.5552 & 1.771194 & -0.067 \\
\hline
\end{tabular}

W $\quad 2.949600 \quad-2.556732 \quad-0.589630$

W $\quad-0.665054 \quad-5.822708 \quad 0.855209$

W $\quad-2.436844 \quad-5.339676 \quad-2.126877$

$\begin{array}{llll}\mathrm{O} & 0.882324 & 3.552307 & -2.067627\end{array}$

$\begin{array}{llll}O & -2.784515 & -1.080307 & 2.705170\end{array}$

$\begin{array}{llll}\mathrm{O} & 2.203354 & 3.217056 & 0.141266\end{array}$

$\begin{array}{llll}\mathrm{O} & -0.685135 & 0.431305 & 3.421829\end{array}$

$\begin{array}{lllll}\mathrm{O} & -3.649582 & -1.878784 & -2.274994\end{array}$

$\begin{array}{llll}0 & -0.671417 & 1.291443 & -1.499634\end{array}$

$\begin{array}{llll}\text { O } & 0.941635 & -0.009979 & -3.674484\end{array}$

$\begin{array}{llll}\mathrm{O} & -3.679352 & 1.303436 & -1.711273\end{array}$

$\begin{array}{llll}\mathrm{O} & -4.145416 & -3.036118 & 1.306122\end{array}$

$\begin{array}{llll}O & -2.291656 & 0.002304 & -3.638077\end{array}$

$\begin{array}{llll}O & -1.956543 & -2.247955 & 0.153030\end{array}$

$\begin{array}{llll}O & -2.310135 & -3.849274 & -3.366913\end{array}$

$\begin{array}{llll}\mathrm{O} & 2.797089 & 1.208801 & 2.060577\end{array}$

$\begin{array}{llll}\text { O } & -0.675323 & -5.979019 & -2.842941\end{array}$

$\begin{array}{llll}\mathrm{O} & -0.713882 & 2.218719 & -3.905228\end{array}$

$\begin{array}{llll}\text { O } & 3.106262 & -0.630814 & -0.025787\end{array}$

$\begin{array}{llll}\mathrm{O} & -4.403122 & -0.601807 & 0.000931\end{array}$

\begin{tabular}{llll}
\hline & -4.171921 & 1.204880 & 2.056046 \\
\hline & -0.665070 & -2.018539 & -4.652893
\end{tabular}

$\begin{array}{llll}\mathrm{O} & -0.665070 & -2.018539 & -4.652893 \\ \mathrm{O} & -0.656219 & -1.882217 & -2.082306\end{array}$

$\begin{array}{llll}\mathrm{O} & -1.965210 & 0.986145 & 0.742538\end{array}$

$\begin{array}{llll}O & 2.834473 & -3.030930 & 1.280884\end{array}$

$\begin{array}{llll}O & 0.660400 & -4.705414 & 1.654495\end{array}$

$\begin{array}{llll}\text { O } & 2.304916 & -1.869019 & -2.286591\end{array}$

$\begin{array}{lllll}O & 0.658615 & -2.260437 & 0.138687\end{array}$

$\begin{array}{llll}O & -1.988602 & -6.359436 & -0.530319\end{array}$

$\begin{array}{llll}\mathrm{O} & -3.687225 & 3.199905 & 0.097961\end{array}$

$\begin{array}{llll}\text { O } & -3.638168 & -4.229446 & -1.134918\end{array}$

$\begin{array}{lllll}\mathrm{O} & 0.691269 & -6.377686 & -0.531586\end{array}$

$\begin{array}{llll}O & 0.995544 & -3.878204 & -3.384933\end{array}$

$\begin{array}{llll}\mathrm{O} & -0.608047 & 3.122818 & 0.330093\end{array}$

$\begin{array}{llll}\text { O } & 2.305908 & -4.265152 & -1.179794\end{array}$

$\begin{array}{lllll}\mathrm{P} & -0.659058 & 1.480118 & 0.054886\end{array}$

$\begin{array}{llll}\mathrm{P} & -0.657074 & -2.574142 & -0.668198\end{array}$

$\begin{array}{lllll}\mathrm{O} & 0.643097 & 0.922119 & 0.728928\end{array}$

$\begin{array}{lllll}\mathrm{O} & -3.536987 & 2.417206 & -4.374481\end{array}$

$\begin{array}{lllll}O & 2.121506 & -2.070526 & -5.173035\end{array}$

$\begin{array}{llll}0 & -0.662613 & -4.177383 & -0.936630 \\ 0 & 2.206192 & 1.321091 & -1.673676\end{array}$

$\begin{array}{llll}\mathrm{O} & 0.708069 & 2.693298 & 3.067947\end{array}$

$\begin{array}{llll}O & -2.113846 & 2.676727 & 3.107239\end{array}$

$\begin{array}{llll}\text { O } & -3.465683 & -2.034988 & -5.152908\end{array}$

$\begin{array}{llll}\text { O } & 1.452026 & -1.070755 & 2.717789\end{array}$

$\begin{array}{llll}O & -2.356247 & 3.530685 & -2.097977\end{array}$

$\begin{array}{lllll}\mathrm{O} & -3.564133 & 5.929550 & -0.988708\end{array}$

$\begin{array}{llll}O & -1.979706 & -4.720993 & 1.678192\end{array}$

$\begin{array}{llll}\text { O } & -3.517380 & -6.452179 & -2.950546\end{array}$

$\begin{array}{llll}\text { O } & -0.657776 & -2.768921 & 2.803894\end{array}$

$\begin{array}{lllll}\text { O } & 4.658478 & -2.812149 & -0.875641\end{array}$

$\begin{array}{llll}\mathrm{O} & 1.863831 & 0.831207 & 4.761597\end{array}$

$\begin{array}{llll}\mathrm{O} & -3.261749 & 0.776993 & 4.750687\end{array}$

$\begin{array}{lllll}\mathrm{O} & -6.021774 & 1.694489 & -0.032288\end{array}$

$\begin{array}{lllll}O & 2.231613 & 5.964310 & -1.016830\end{array}$

$\begin{array}{lllll}O & 2.084763 & 2.476883 & -4.308823\end{array}$

O $\quad 2.113602 \quad-6.541183 \quad-2.994263$

$\begin{array}{lllll}\text { O } & 4.555237 & 1.771194 & -0.067535\end{array}$ 


$\begin{array}{rrrr}\mathrm{O} & -3.195938 & -3.556900 & 3.929855 \\ \mathrm{O} & -0.654709 & -7.266037 & 1.856491 \\ \mathrm{O} & 1.893341 & -3.555756 & 3.904709 \\ \mathrm{C} & -0.715027 & 5.400681 & 4.266724 \\ \mathrm{H} & 0.175369 & 6.062088 & 4.205505 \\ \mathrm{H} & -0.666718 & 4.762314 & 5.173035 \\ \mathrm{H} & -1.647476 & 6.004837 & 4.263428 \\ \mathrm{C} & 3.342453 & 6.144302 & -1.741333 \\ \mathrm{O} & 3.821182 & 7.266037 & -1.903885 \\ \mathrm{C} & 3.957733 & 4.882263 & -2.331253 \\ \mathrm{C} & 5.235107 & 5.145584 & -3.131134 \\ \mathrm{H} & 3.189804 & 4.373581 & -2.960297 \\ \mathrm{H} & 4.142914 & 4.158188 & -1.504150 \\ \mathrm{H} & 5.632828 & 4.191986 & -3.543518 \\ \mathrm{H} & 5.041946 & 5.843719 & -3.977356 \\ \mathrm{H} & 6.021774 & 5.613342 & -2.495758\end{array}$

\subsubsection{Conf5}

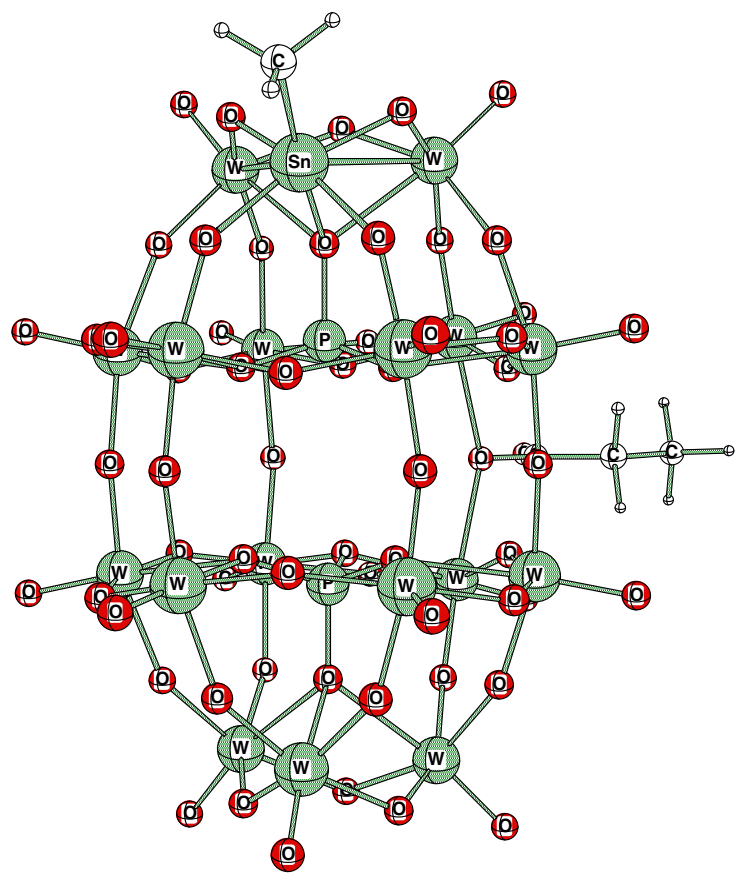

94

Energy $=-6650.3390102550$

$\begin{array}{cccc}\mathrm{O} & -2.635651 & 5.449188 & 0.829529 \\ \mathrm{Sn} & -1.429047 & 4.575729 & 2.332413 \\ \mathrm{O} & 0.265030 & 5.492935 & 1.396713 \\ \text { W } & 0.826096 & 4.795212 & -0.241913 \\ \mathrm{O} & 2.021851 & 5.988861 & -0.719620 \\ \mathrm{O} & -0.776077 & 5.410751 & -1.157043 \\ \text { W } & -2.617172 & 4.700760 & -0.910461 \\ \text { W } & -2.224075 & 1.600723 & -3.084747 \\ \text { W } & 1.223251 & 1.824280 & -2.535797 \\ \text { W } & 1.257751 & -2.521774 & -2.680847 \\ \text { W } & 2.458099 & 1.524857 & 0.981628 \\ \text { W } & 0.269501 & 1.462662 & 3.700500 \\ \text { W } & -2.175629 & -2.242111 & -3.226852 \\ \text { W } & -3.430206 & 1.454971 & 3.104614 \\ \text { W } & 0.326416 & -2.394333 & 3.531036 \\ \text { W } & -3.408783 & -2.386017 & 2.928024 \\ \text { W } & -4.610764 & 1.518753 & -0.186310\end{array}$

\begin{tabular}{|c|c|c|c|}
\hline & 1 & & \\
\hline & 0.871979 & -5.543854 & -0.475006 \\
\hline & 2.507019 & -2.328079 & 0.841049 \\
\hline & -1.391891 & -5.481232 & $2.1466 \mathrm{~s}$ \\
\hline & -2.531296 & -5.410706 & -1.1668 \\
\hline & 0.945251 & 3.502838 & -1.83523 \\
\hline & -3.634476 & -0.517960 & 2.89093 \\
\hline & 1.874557 & 3.370697 & 0.58316 \\
\hline & -1.627670 & 1.070160 & 3.742554 \\
\hline & -3.657150 & -2.027740 & $-2.0104 C$ \\
\hline & -0.764786 & 1.289017 & $-1.1654 \mathrm{C}$ \\
\hline & 1.701782 & -0.345078 & -3.279373 \\
\hline & -3.677414 & 1.239243 & -1.889740 \\
\hline & -4.784149 & -2.648346 & 1.569031 \\
\hline & -2.035019 & -0.338806 & $-3.3322 \varsigma$ \\
\hline & -2.414047 & -2.040131 & 0.685684 \\
\hline & -2.159546 & -4.109833 & -2.550613 \\
\hline & 2.065430 & 1.667236 & 2.849503 \\
\hline & -0.707809 & -6.173721 & -1.411011 \\
\hline & -0.332413 & 1.766525 & -3.649750 \\
\hline & 2.569092 & -0.419754 & 1.086395 \\
\hline & -4.746780 & -0.430359 & -0.09944 \\
\hline & -4.827576 & 1.649597 & 1.726425 \\
\hline & -0.295349 & -2.444153 & -3.801590 \\
\hline & -0.714920 & -1.999374 & -1.316956 \\
\hline & -2.452698 & 1.210114 & $0.8358 s$ \\
\hline & 2.095154 & -2.651749 & 2.694305 \\
\hline & -0.167145 & -4.257141 & 2.965317 \\
\hline & 2.152390 & -2.009186 & -1.074875 \\
\hline & 0.155579 & -2.034485 & 1.137497 \\
\hline & -2.430435 & -6.193726 & 0.637787 \\
\hline & -3.927048 & 3.352051 & -0.3 \\
\hline & -3.875549 & -4.179474 & -0.558029 \\
\hline & 0.211075 & -6.247314 & 1.150406 \\
\hline & 1.005402 & -4.2 & -2.039749 \\
\hline & -1.055634 & 3.322540 & 0.418640 \\
\hline & 1.932098 & -4.178802 & 0.382507 \\
\hline & -1.043915 & 1.676147 & 0.332397 \\
\hline & -0.998245 & -2.47 & 3361 \\
\hline & 0.114700 & 1.205933 & 1.281601 \\
\hline & -3.026962 & 1.962997 & -4.596130 \\
\hline & 2.535019 & -2.911728 & -3.817352 \\
\hline & -0.988281 & -4.100784 & 305 \\
\hline & 2.105225 & 1.241547 & -0.954758 \\
\hline & -0.130249 & 3.258728 & 3.331192 \\
\hline & -2.945694 & 3.254105 & \\
\hline & -2.974350 & -2.564148 & -4.750244 \\
\hline & 0.557755 & -0.519241 & 3.588531 \\
\hline & -2.207672 & 3.388855 & -2.342743 \\
\hline & -3.566956 & 5.894745 & -1.77798 \\
\hline & -2.782043 & -4.267303 & 2.56280 \\
\hline & -3.473892 & -6.637650 & -1.997086 \\
\hline & -1.597870 & -2.168549 & 3.585480 \\
\hline & 4.228439 & -2.650269 & 0.85154 \\
\hline & 0.704147 & 1.630020 & 5.390213 \\
\hline & -4.375092 & 1.590179 & 4.574066 \\
\hline & -6.251251 & 1.862411 & -0.689713 \\
\hline & 2.500137 & 2.263947 & -3.654373 \\
\hline & -6.223999 & -2.680771 & -0.869934 \\
\hline & 2.061111 & -6.751831 & $-U$. \\
\hline
\end{tabular}




$\begin{array}{rrrr}\mathrm{O} & 4.175751 & 1.862961 & 1.010956 \\ \mathrm{O} & -4.316803 & -2.773972 & 4.372696 \\ \mathrm{O} & -1.588501 & -6.751343 & 3.343750 \\ \mathrm{O} & 0.720779 & -2.796555 & 5.189148 \\ \mathrm{C} & -1.734161 & 6.062866 & 3.897034 \\ \mathrm{H} & -0.863159 & 6.751831 & 3.908798 \\ \mathrm{H} & -1.833115 & 5.535477 & 4.868668 \\ \mathrm{H} & -2.666611 & 6.618561 & 3.660965 \\ \mathrm{C} & 2.732468 & -0.305450 & -4.182953 \\ \mathrm{O} & 2.572235 & -0.270737 & -5.390213 \\ \mathrm{C} & 4.121216 & -0.310501 & -3.533127 \\ \mathrm{C} & 5.258179 & -0.241776 & -4.554871 \\ \mathrm{H} & 4.198593 & -1.224548 & -2.905899 \\ \mathrm{H} & 4.165543 & 0.542847 & -2.823868 \\ \mathrm{H} & 6.251236 & -0.215790 & -4.045242 \\ \mathrm{H} & 5.233765 & -1.124603 & -5.232620 \\ \mathrm{H} & 5.162201 & 0.668213 & -5.188705\end{array}$

\subsection{7 $\underline{\text { Conf6 }}$}

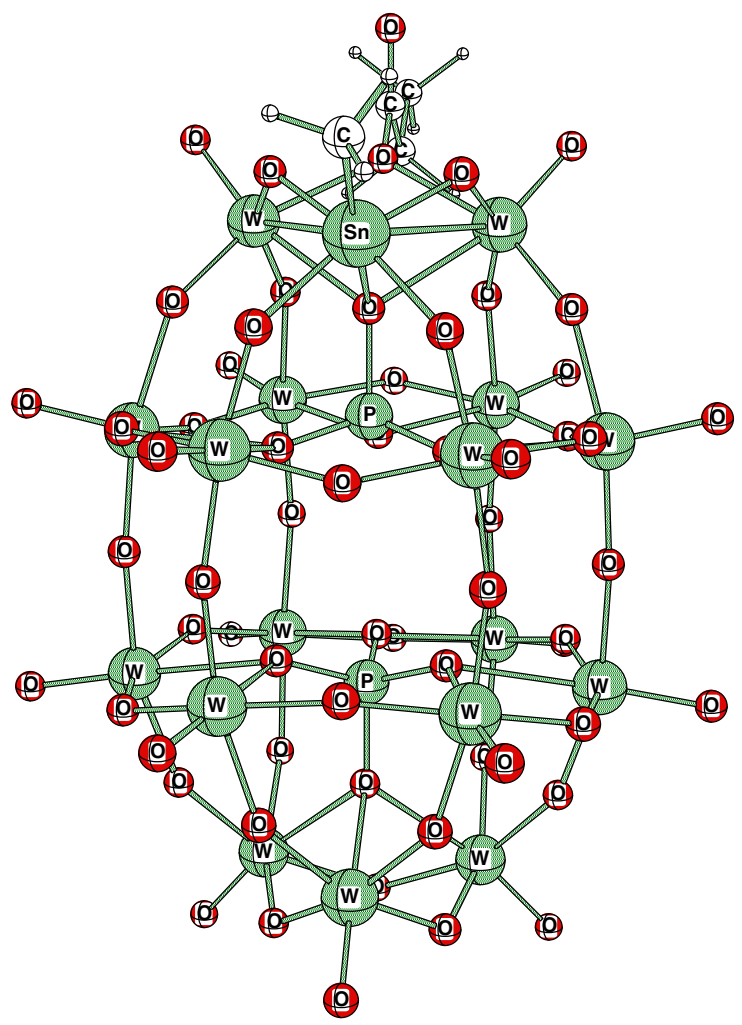

94

\begin{tabular}{lrrr}
\multicolumn{4}{c}{ Energy $=-6650.3635748910$} \\
Sn & -0.192352 & 3.933670 & 2.671555 \\
O & 1.402283 & 4.897171 & 1.678024 \\
W & 2.025192 & 4.243683 & 0.024704 \\
O & 0.220581 & 5.343872 & -0.836334 \\
W & -1.786224 & 4.339371 & -0.381027 \\
O & -1.487137 & 4.973251 & 1.367966 \\
W & -1.397308 & 1.619583 & -2.975464 \\
W & 2.045731 & 1.539078 & -2.616287 \\
W & 2.038986 & -2.259277 & -3.253082 \\
W & 3.614532 & 0.891205 & 0.782898 \\
W & 1.518921 & 0.641769 & 3.567917 \\
W & -1.412888 & -2.176315 & -3.632019
\end{tabular}

\begin{tabular}{|c|c|c|c|}
\hline & 39 & 918 & \\
\hline & & -3.167496 & \\
\hline & -2.258530 & -3.076080 & 86481 \\
\hline & -3.662170 & 1.071136 & .024 \\
\hline & -3.656586 & -2.706039 & 000 \\
\hline & 1.770111 & -5.702377 & -1.649521 \\
\hline & 3.562927 & -2.888504 & 1210 \\
\hline & -0.280365 & -6.034668 & 1.1683 \\
\hline & -1.668930 & -5.618256 & $-2.0191 s$ \\
\hline & 1.865952 & 3.240067 & -1.631577 \\
\hline & -2.462326 & -1.221527 & $8<0$ \\
\hline & 3.094208 & 2.900284 & 0.71426 \\
\hline & -0.382797 & 0.244843 & 3.64660 \\
\hline & -2.826309 & -2.075821 & -2.29393 \\
\hline & 0.145462 & 1.036 & -1.21 \\
\hline & 1.946213 & -0.314194 & -3.1348 \\
\hline & -2.782852 & 1.148941 & -1.703018 \\
\hline & -3.737534 & -3.188309 & 1.205963 \\
\hline & -1.268784 & -0.236130 & -3.479 \\
\hline & -1.422302 & -2.476929 & $0.2936 \xi$ \\
\hline & -1.432770 & -4.086884 & -3.222412 \\
\hline & 3.262848 & 0.904068 & 2.679 \\
\hline & 0.112167 & -6.243637 & -2.5380 \\
\hline & 0.418976 & 1.948624 & -3.609451 \\
\hline & 3.672058 & 5916 & 0.678024 \\
\hline & -3.787064 & -0.800964 & -0.119 \\
\hline & -3.710632 & 1.071533 & 1.9469 \\
\hline & 0.405899 & -2.291290 & -4.320190 \\
\hline & 0.130157 & -2.145096 & -1.77 \\
\hline & -1.391296 & 0.798965 & 0.877472 \\
\hline & 3.218582 & -3.3 & 1.961 \\
\hline & 0.976715 & 4203 & 2.101883 \\
\hline & 3.124496 & -2.221680 & -1.642197 \\
\hline & 1.183228 & -2.540146 & 0.578049 \\
\hline & -1.473267 & -6.5 & -0.368423 \\
\hline & -3.044144 & 3.0 & 0.067032 \\
\hline & -2.998688 & -4.453613 & -1.171722 \\
\hline & 1.181702 & -6.643829 & -0.082031 \\
\hline & 947 & -4.1 & 79 \\
\hline & 0.009186 & 2.9 & 0.5 \\
\hline & 2.943970 & -4.601410 & -0.528793 \\
\hline & -0.006866 & 1.290253 & 0.328018 \\
\hline & -0.039276 & -2.8 & -0.3 \\
\hline & 1.210541 & 0.733734 & 1.144394 \\
\hline & -2.312408 & 2.215134 & -4.343979 \\
\hline & 3.233459 & -2.380615 & -4.527435 \\
\hline & -0.049789 & -4.433472 & -0.630844 \\
\hline & 3.120132 & 1.003769 & -1.085587 \\
\hline & 1.111633 & 2.468781 & 3.448181 \\
\hline & -1.700439 & 2.538956 & 3.155045 \\
\hline & -2.301987 & -2.249191 & -5.138275 \\
\hline & 1.753204 & -1.322937 & 3.177750 \\
\hline & -1.332474 & 3.321442 & -1.969864 \\
\hline & -2.728760 & 5.659348 & -1.056412 \\
\hline & -1.653885 & -4.897537 & 1.812851 \\
\hline & -2.672470 & -6.714020 & -2.95372 \\
\hline & -0.412354 & -2.968414 & 3.0600 \\
\hline & 5.283188 & -3.191315 & 0.02279 \\
\hline & 2.020828 & 0.571030 & 5.2449 \\
\hline & -3.037 & 0.695419 & $4.71 \mathrm{~s}$ \\
\hline
\end{tabular}




$\begin{array}{rrrr}\mathrm{O} & -5.320526 & 1.496078 & -0.336685 \\ \mathrm{O} & 3.158188 & 5.505905 & -0.434509 \\ \mathrm{O} & 3.241150 & 2.082077 & -3.774460 \\ \mathrm{O} & -5.329239 & -2.927567 & -1.130127 \\ \mathrm{O} & 2.892410 & -6.853439 & -2.353149 \\ \mathrm{O} & 5.329239 & 1.238373 & 0.772415 \\ \mathrm{O} & -3.070679 & -3.676773 & 3.915955 \\ \mathrm{O} & -0.419540 & -7.470734 & 2.170303 \\ \mathrm{O} & 1.947556 & -3.786407 & 4.471191 \\ \mathrm{C} & -0.371262 & 5.220459 & 4.419403 \\ \mathrm{H} & 0.297684 & 6.095901 & 4.277161 \\ \mathrm{H} & -0.074646 & 4.642288 & 5.319275 \\ \mathrm{H} & -1.429047 & 5.549194 & 4.499451 \\ \mathrm{C} & 0.350372 & 6.264145 & -1.825760 \\ \mathrm{O} & 0.340927 & 7.470718 & -1.615723 \\ \mathrm{C} & 0.506622 & 5.668304 & -3.217178 \\ \mathrm{C} & 0.649521 & 6.712418 & -4.325378 \\ \mathrm{H} & -0.359818 & 4.990128 & -3.385956 \\ \mathrm{H} & 1.371841 & 4.968781 & -3.184799 \\ \mathrm{H} & 0.752487 & 6.218704 & -5.319275 \\ \mathrm{H} & -0.236099 & 7.387482 & -4.357971 \\ \mathrm{H} & 1.543289 & 7.356232 & -4.159042\end{array}$

\subsection{8 $\underline{\text { Conf7 }}$}

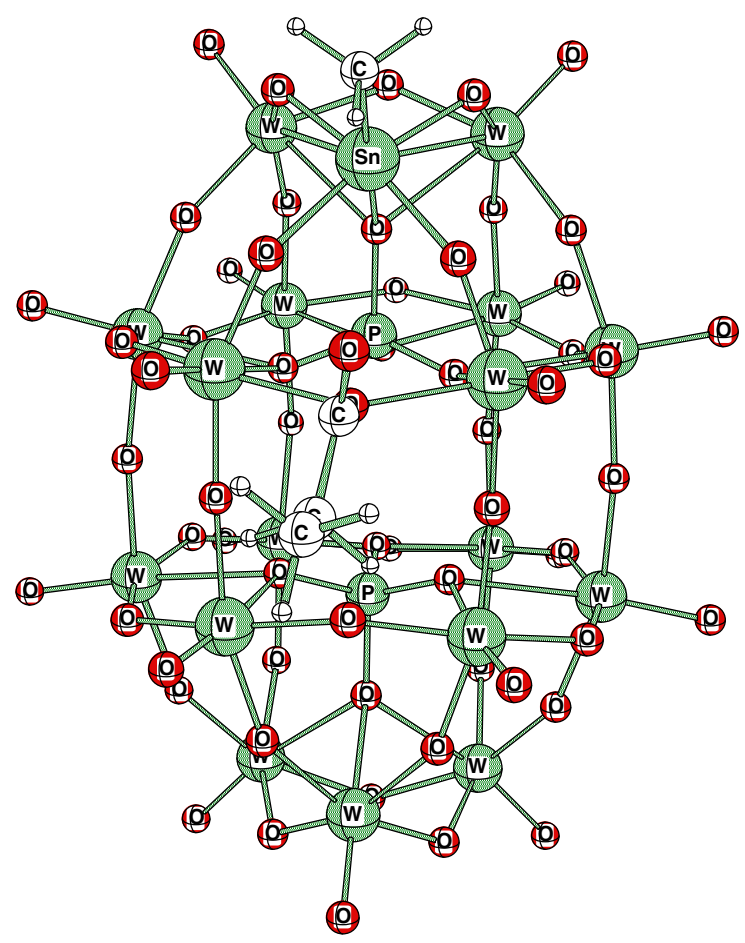

94

\begin{tabular}{lccc}
\multicolumn{4}{c}{ Energy $=-6650.3429247950$} \\
W & -1.566467 & 5.337311 & -1.637375 \\
O & -1.420471 & 5.750137 & 0.197266 \\
Sn & -0.072556 & 4.611069 & 1.389648 \\
O & 1.521271 & 5.641724 & 0.428787 \\
W & 1.927109 & 5.201904 & -1.360138 \\
O & 0.244827 & 6.014313 & -1.974045 \\
W & -1.433655 & 2.674896 & -4.381287 \\
W & 2.023895 & 2.538391 & -4.098312 \\
W & 1.928253 & -1.235565 & -4.845230
\end{tabular}

\begin{tabular}{|c|c|c|c|}
\hline & 59 & & \\
\hline W & 1.828644 & 1.347122 & .092209 \\
\hline Wh & -1.525604 & 103287 & \\
\hline W & -2.320404 & 1.500427 & 1.73054 \\
\hline & 1.540100 & -2.434937 & 1.2926 \\
\hline W & -2.203659 & -2.303497 & 0.985886 \\
\hline Wh & -3.620544 & 2.131180 & -1.407700 \\
\hline $\mathrm{W}$ & -3.690445 & -1.649017 & -2.084717 \\
\hline & 1.679214 & -4.700027 & -3.313660 \\
\hline & 3.534103 & -1.935928 & -1.508026 \\
\hline & -0.304535 & -5.221100 & -0.49923 \\
\hline W & -1.791107 & -4.565643 & -3.58200 \\
\hline & 1.910553 & 4.140701 & -3.039368 \\
\hline & -2.315964 & -0.387619 & 1.338654 \\
\hline & 3.042648 & 3.689255 & -0.7 \\
\hline & -0.316498 & 0.946869 & 2.806381 \\
\hline & -2.890427 & -1.046875 & -3.735992 \\
\hline & 0.137009 & 1.9 & 078 \\
\hline & 1.860916 & 0.677887 & -4.6860 \\
\hline & -2.841522 & 2.104034 & -3.14608 \\
\hline & -3.721848 & 1039 & -0.2 \\
\hline & -1.330841 & 0.803726 & -4.93809 \\
\hline & -1.427612 & -1.550568 & -1.17523 \\
\hline & -1.550079 & 5624 & 1007 \\
\hline 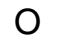 & 3.446365 & 1.6 & 1.1 \\
\hline & -0.028000 & -5.245026 & -4.12155 \\
\hline & 0.371460 & & -5.0 \\
\hline & 680 & -0.0 & $-0 . \varsigma$ \\
\hline & -3.794495 & 417 & -1.51667 \\
\hline 0 & -3.746231 & 1.945648 & 0.585419 \\
\hline & 0.268280 & -1.2 & 368 \\
\hline & 0.067383 & -1.1 & -3.2 \\
\hline & -1.363861 & 356 & -0.4 \\
\hline & 3.225983 & -2.5 & 0.3 \\
\hline & 0.9 & -4 & \\
\hline & 3.054504 & -1.2 & -3.2 \\
\hline & 1.175446 & -1.650818 & -0.96618 \\
\hline & -1.564117 & -5.6 & -1.5 \\
\hline & $-2.8 \varepsilon$ & 46 & -1.2 \\
\hline & -3.045471 & -3.424042 & $-2.63906 s$ \\
\hline & 1.114426 & -5.741943 & -1.737000 \\
\hline $\mathrm{O}$ & 1.74 & -3.1 & 366 \\
\hline & 0.05 & 12 & -0.7 \\
\hline & 2.849915 & -3.66 & -2.169846 \\
\hline & 0.017426 & $2.0 \varsigma$ & -1.039124 \\
\hline & -0.07 & -1.8 & \\
\hline & 1.24 & 1.47 & -0.2 \\
\hline & -2.345306 & 3.308044 & -5.7 \\
\hline & 3.083099 & -1.3 & -6.1 \\
\hline & -0.107925 & -3.4 & -2.2 \\
\hline & 3.152924 & 1.86 & -2.65 \\
\hline & 1.245117 & 3.107162 & 2.04884 \\
\hline & -1.568741 & 3.194565 & 1.814606 \\
\hline & -2.465912 & -1.148254 & -6.594345 \\
\hline & 1.748093 & -0.542343 & 1.67311 \\
\hline & -1.370468 & 4.264603 & -3.300125 \\
\hline & -2.560791 & 6.671646 & -2.19564 \\
\hline & -1.651855 & -4.044693 & רדרי 0 \\
\hline & -2.845734 & -5.615097 & -4.51391 \\
\hline & & 306 & \\
\hline
\end{tabular}




$\begin{array}{rrrr}O & 5.237473 & -2.302933 & -1.674667 \\ O & 2.468796 & 1.239975 & 3.715958 \\ O & -3.185364 & 1.455688 & 3.254700 \\ O & -5.279434 & 2.574875 & -1.737442 \\ O & 3.097504 & 6.451935 & -1.745697 \\ O & 3.189667 & 3.087677 & -5.281540 \\ O & -5.370331 & -1.881393 & -2.518234 \\ O & 2.782333 & -5.836121 & -4.071899 \\ O & 5.370346 & 2.153900 & -0.876938 \\ O & -3.004120 & -2.872772 & 2.438293 \\ \text { O } & -0.440567 & -6.671661 & 0.481750 \\ \text { O } & 2.046646 & -3.066666 & 2.847366 \\ \mathrm{C} & -0.183136 & 5.739212 & 3.244446 \\ \mathrm{H} & 0.729477 & 6.366684 & 3.329834 \\ \mathrm{H} & -0.236588 & 5.005035 & 4.074677 \\ \mathrm{H} & -1.093704 & 6.374802 & 3.217926 \\ \mathrm{C} & -0.470322 & 0.778702 & 4.150848 \\ \mathrm{O} & -0.324768 & 1.672577 & 4.971725 \\ \mathrm{C} & -0.824753 & -0.649963 & 4.547211 \\ \mathrm{C} & -0.981430 & -0.842087 & 6.057465 \\ \mathrm{H} & -0.037018 & -1.315796 & 4.130478 \\ \mathrm{H} & -1.751419 & -0.939743 & 4.006958 \\ \mathrm{H} & -1.228134 & -1.903381 & 6.294250 \\ \mathrm{H} & -0.045212 & -0.568634 & 6.594330 \\ \mathrm{H} & -1.792267 & -0.192902 & 6.458572\end{array}$

\subsection{9 $\underline{\text { Conf8 }}$}

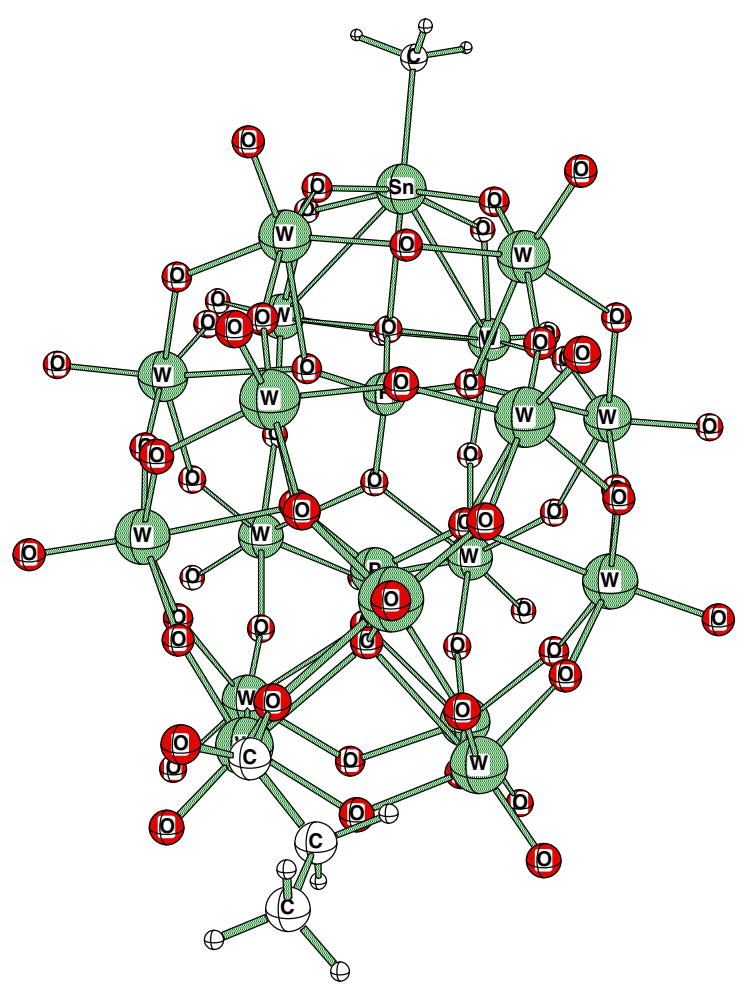

94

Energy $=-6650.3606772980$

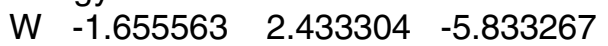

$\begin{array}{llll}\text { O } & -1.205048 & 4.186661 & -5.317825\end{array}$

$\begin{array}{lllll}\text { Sn } & 0.364136 & 4.451477 & -3.912720\end{array}$

$\begin{array}{lllll}O & 1.733139 & 3.883026 & -5.424973\end{array}$

W $1.838196 \quad 2.060852 \quad-5.921677$

\begin{tabular}{|c|c|c|c|}
\hline & & & \\
\hline & 240 & 4167 & 500 \\
\hline & 1.496704 & -1.593262 & -4.903748 \\
\hline & 1.348083 & -4.133011 & -2.006210 \\
\hline & 3.662033 & 0.691666 & -2.85835 \\
\hline & 2.254471 & 3.203049 & -0.879333 \\
\hline & -2.107620 & -3.784180 & -2.00407 \\
\hline W & -1.475327 & 3.507706 & -0.717102 \\
\hline WV & 2.169998 & 0.659515 & 2.01728 \\
\hline N & -1.623947 & 0.933975 & 2.177414 \\
\hline is & -3.476669 & 1.375534 & -2.632431 \\
\hline I & -3.628387 & -1.153015 & 0.28680 \\
\hline 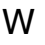 & 1.481720 & -4.535110 & 1.764221 \\
\hline W & 3.544968 & -1.847855 & 0.043930 \\
\hline N & 0.160629 & -1.988800 & 3.772583 \\
\hline I & -2.012711 & -4.259872 & 1.71783 \\
\hline O & 1.527405 & 0.109436 & -5.817780 \\
\hline 0 & -1.772049 & 2.170456 & 0.777420 \\
\hline & 972 & 1.687073 & -4 \\
\hline 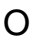 & 0.417297 & 3.3 & -0.2 \\
\hline 0 & -3.151749 & -2.446976 & 97244 \\
\hline 0 & -0.083557 & 676 & 0329 \\
\hline 0 & 1.255737 & -3.008698 & -3.553696 \\
\hline 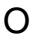 & -3.037933 & -0.280869 & 3187 \\
\hline $\mathrm{O}$ & -3.327744 & 8827 & 4990 \\
\hline 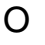 & -1.917419 & -2.7 & 631 \\
\hline 0 & -1.280334 & -0.6 & 0.410904 \\
\hline $\mathrm{O}$ & -2.055557 & b247 & -0.086517 \\
\hline 0 & 426 & 2.2 & 0778 \\
\hline 0 & -0.34 & -5.2 & 4145 \\
\hline 0 & -0.309814 & -1.943817 & -5.571594 \\
\hline 0 & 3.725098 & 639 & 4196 \\
\hline 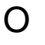 & -3.657532 & 77 & 99 \\
\hline 0 & -3.188599 & 265 & 3719 \\
\hline $\mathrm{O}$ & -0.472794 & -4.77 & -2.320480 \\
\hline 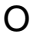 & -0.2 & & 018 \\
\hline 0 & -1.1 & 08 & 21 \\
\hline $\mathrm{O}$ & 3.595810 & -0.5 & 4333 \\
\hline $\mathrm{O}$ & 1.542999 & -0.8 & 8929 \\
\hline & & & -1.3 \\
\hline 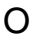 & 068 & $-0 . c^{-1}-x$ & 0.3 \\
\hline 0 & -1.550430 & -3.4 & 861 \\
\hline 0 & -2.8 & & -4.2 \\
\hline & -3.0 & & \\
\hline $\mathrm{O}$ & 334 & -3.6 & 3.4 \\
\hline $\mathrm{O}$ & 1.265411 & -4.75 & -0.134247 \\
\hline 0 & 0.1 & & 208 \\
\hline & 2.7 & -3 & \\
\hline 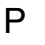 & 0.08 & 1.0 & -2.8 \\
\hline$P$ & -0.086533 & 638 & 0092 \\
\hline $\mathrm{O}$ & & & -2.1 \\
\hline & -3.144135 & -1.9 & -5.9 \\
\hline 0 & 2.255112 & -5.475708 & -2.665085 \\
\hline O & -0.187851 & -2.723755 & 1.516998 \\
\hline & 2.844376 & -0.8 & -3.7 \\
\hline $\mathrm{O}$ & 1.831131 & & -2.432 \\
\hline $\mathrm{O}$ & -1.001785 & 4.367966 & -2.310959 \\
\hline & -3.309509 & -4.922592 & -2.571625 \\
\hline 0 & & & 0.58815 \\
\hline & -1.7494 & 0.438614 & -57528 \\
\hline
\end{tabular}




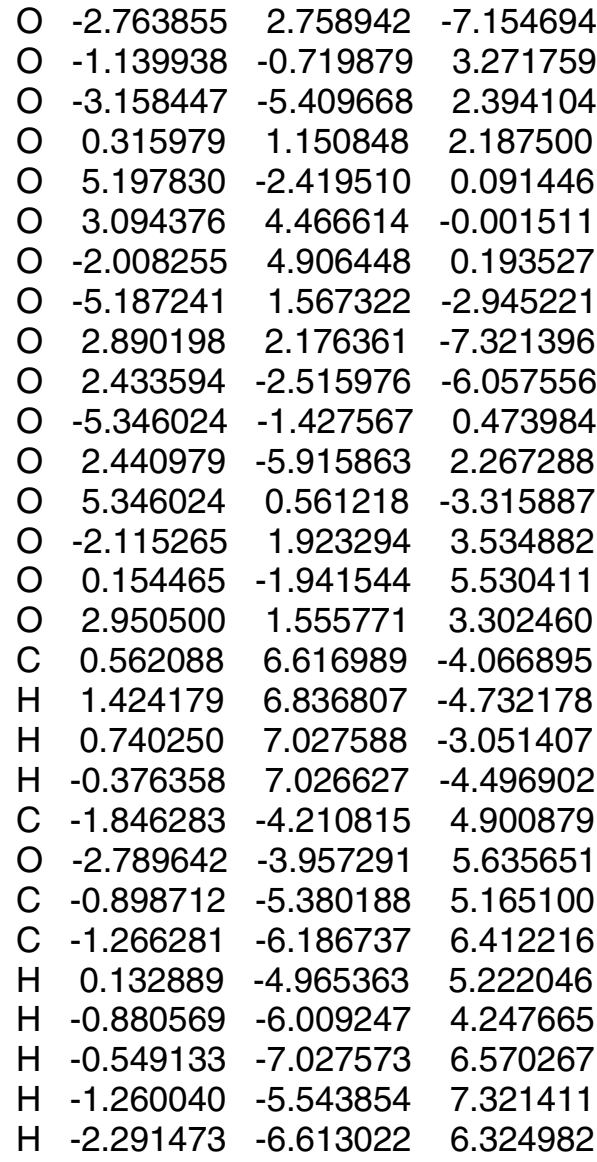

1.6.10 $\underline{\text { Conf9 }}$

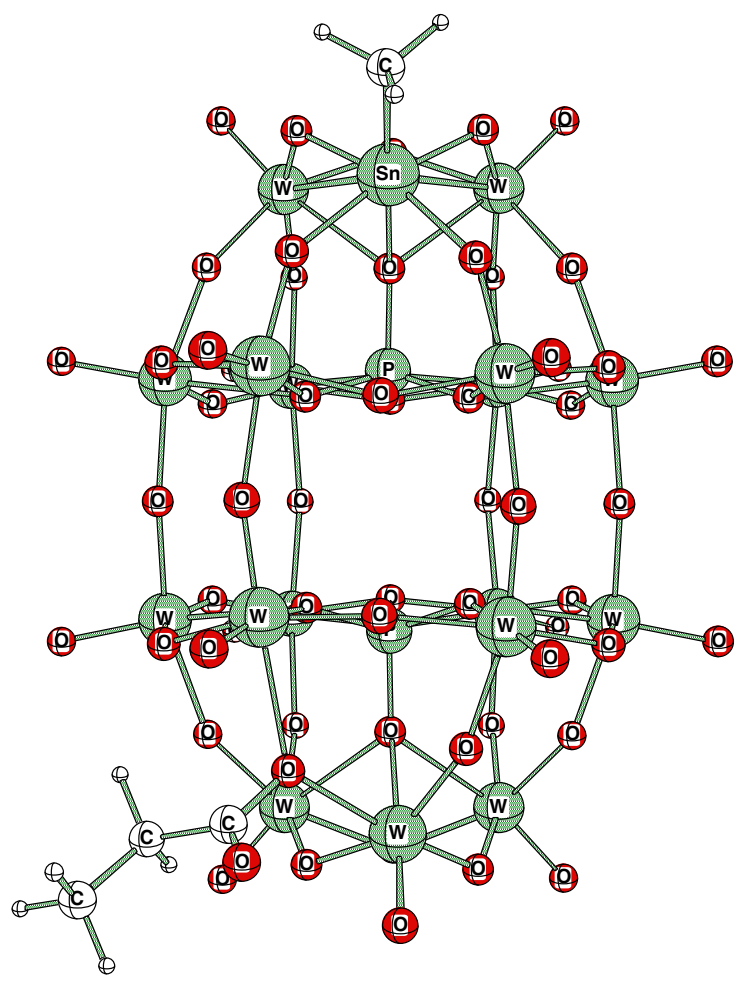

94

Energy $=-6650.3543213010$

W $2.004288 \quad-2.018112 \quad-3.290390$

\begin{tabular}{|c|c|c|c|}
\hline & 901 & 162 & \\
\hline & 9922 & -2.077896 & 48584 \\
\hline & 3.582306 & -2.385696 & 1.979080 \\
\hline W & 1.969254 & -2.075333 & 2.99 \\
\hline & 0.025345 & -1.900269 & 3.2796 \\
\hline W & -1.551956 & 4.952408 & -1.159912 \\
\hline & -1.299530 & 5.758774 & 0.5327 \\
\hline & 0.147644 & 4.942474 & 1.84400 \\
\hline & 1.660294 & 5.755737 & 0.615997 \\
\hline W & 1.963623 & 4.961456 & -1.072189 \\
\hline & 0.232712 & 5.589462 & -1.7263 \\
\hline & -1.474838 & 1.796829 & -3.29586 \\
\hline & 2.004227 & 1.817444 & -3.20912 \\
\hline & 3.751389 & 1.784760 & 0.106857 \\
\hline & 1.933533 & 1.815887 & \\
\hline & -1.465134 & -2.056122 & -3.3363 \\
\hline & -1.810349 & 1.926483 & 3.001312 \\
\hline & -1.833344 & -1.927368 & 2.939301 \\
\hline & -3.429245 & 1.796722 & -0.09 \\
\hline & -3.438751 & -2.065002 & -0.121964 \\
\hline & 1.977432 & -5.172318 & -1.217102 \\
\hline & 0.303741 & -5.355698 & 1.80548 \\
\hline & -1.528946 & -5.171387 & -1.174622 \\
\hline & 1.895966 & 3.594940 & -2.508514 \\
\hline & -2.091599 & -0.126572 & 2.929764 \\
\hline & 3.135010 & 3.602127 & -0.22963 \\
\hline & 0.031830 & 1.461807 & 3.37867 \\
\hline & -2.749985 & -1.791672 & -1.896606 \\
\hline & 0.203873 & 1.5 & -1.5 \\
\hline & 1.852509 & -0.140671 & -3.44171 \\
\hline & -2.745438 & 1.445312 & -1.8 \\
\hline & -3.357819 & -2.3 & 4331 \\
\hline & -1.330902 & -0.157242 & -3.50807 \\
\hline 0 & -1.145981 & -1.842209 & 0.67091 \\
\hline 0 & -1.395248 & -3.911377 & 1506 \\
\hline & 3.587051 & 1.92 & 2.0 \\
\hline & 0.233902 & -5.906998 & -1.70585 \\
\hline & 0.286514 & 1.994202 & -4.107895 \\
\hline 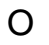 & 3.86 & -0. & 41 \\
\hline & -3.53 & -0.1 & 0.1 \\
\hline 0 & -3.384720 & 2.012680 & 1.84669 \\
\hline & 0.284973 & -2.305527 & -4.149567 \\
\hline & 0.19 & -1.7 & -1.5 \\
\hline & -1.167084 & 006 & 0.6 \\
\hline 0 & 1.350540 & -3.978928 & 2.521210 \\
\hline 0 & 1.462280 & -1.7 & 0.682968 \\
\hline & -1.142639 & -5.9 & 0.6 \\
\hline & -2.789474 & 3.626 & -0.3 \\
\hline & -2.766449 & -3.928009 & -0.385132 \\
\hline & 1.594635 & -6.00 & 0.601776 \\
\hline & 1.899124 & -3.909821 & -2.62287 \\
\hline & 0.173645 & 3.587219 & -0.01736 \\
\hline 0 & 3.128281 & -3.937210 & -0.290482 \\
\hline & 0.161728 & 1.941559 & -0.046875 \\
\hline & 0.180603 & -2.239319 & -0.096466 \\
\hline & 1.440369 & 1.485352 & 0.74266 \\
\hline & -2.499237 & 2.110275 & -4.679810 \\
\hline & 3.096497 & -2.336761 & -4.618 \\
\hline & 0.19 & -3.862579 & -0.117 \\
\hline & & & \\
\hline
\end{tabular}




$\begin{array}{rrrr}\mathrm{O} & 1.508667 & 3.603607 & 2.741180 \\ \mathrm{O} & -1.337372 & 3.692078 & 2.689285 \\ \mathrm{O} & -2.491928 & -2.401245 & -4.710876 \\ \mathrm{O} & 2.140701 & -0.217331 & 3.040192 \\ \mathrm{O} & -1.400375 & 3.589142 & -2.591583 \\ \mathrm{O} & -2.603378 & 6.133560 & -1.919464 \\ \mathrm{O} & -1.388672 & -4.287033 & 2.800354 \\ \mathrm{O} & -2.588608 & -6.416595 & -1.814713 \\ \mathrm{O} & 5.485443 & -2.425446 & -0.132217\end{array}$

$\begin{array}{rrrr}\mathrm{O} & 2.570450 & 2.002182 & 4.710876 \\ \mathrm{O} & -2.540726 & 2.100510 & 4.585632 \\ \mathrm{O} & -5.128052 & 2.105453 & -0.378235 \\ \mathrm{O} & 3.072937 & 6.123917 & -1.776505 \\ \mathrm{O} & 3.111771 & 2.123123 & -4.529373 \\ \mathrm{O} & -5.129501 & -2.418442 & -0.404297 \\ \mathrm{O} & 3.067474 & -6.395767 & -1.844498\end{array}$




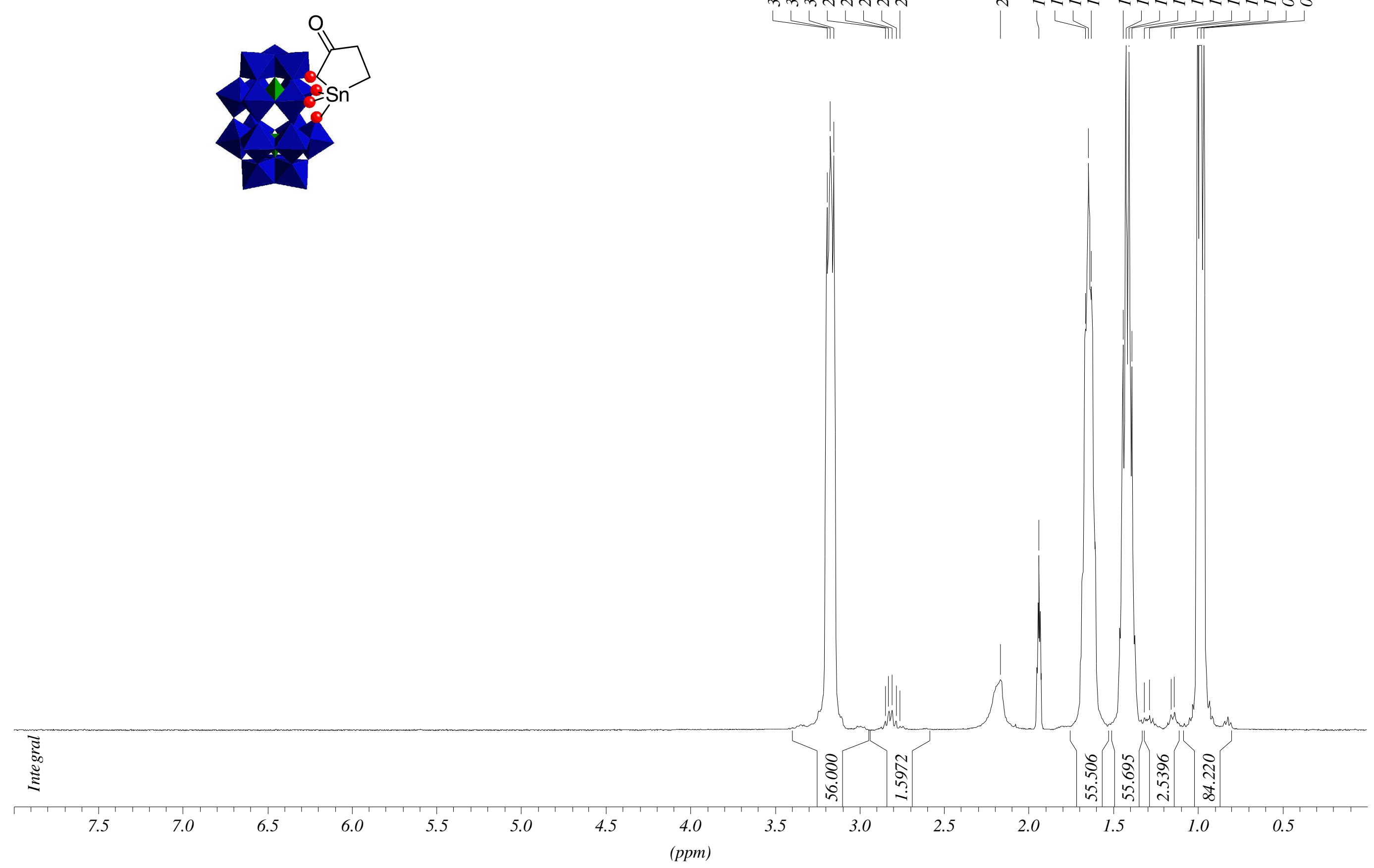



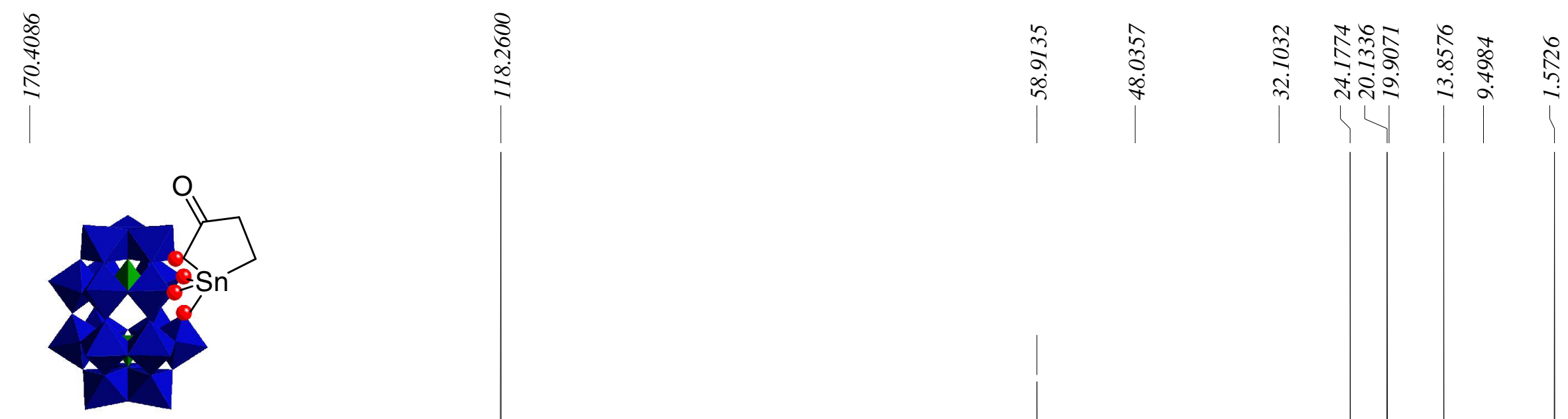


\begin{tabular}{|c|c|c|}
\hline NAME & & $p 2 w 17 s \sim 3$ \\
\hline EXPNO & & 23 \\
\hline PROCNO & & 1 \\
\hline \multicolumn{3}{|c|}{ *** Acquisition } \\
\hline$B F 1$ & . & 111.9203740 \\
\hline$D[1]$ & & $0.2500000 \mathrm{~s}$ \\
\hline$D[33]$ & & $0.0000000 \mathrm{~s}$ \\
\hline$D A T E \_t$ & & 09:58:41 \\
\hline$D A T E \_d$ & & Sep 212006 \\
\hline$N S$ & & 1664 \\
\hline SOLVENT & & $\mathrm{CD} 3 \mathrm{CN}$ \\
\hline$S W$ & & 44.6974 \\
\hline$D$ & & 16384 \\
\hline
\end{tabular}

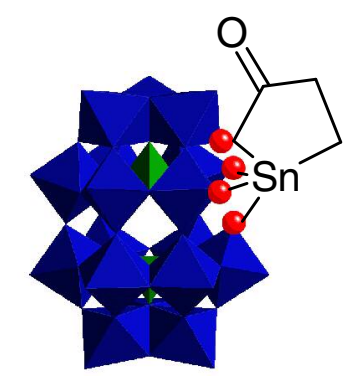

*** Processing Parameters ***

$B C F W \quad: \quad 0.0000000$

*** ID NMR Plot Parameters ***

SOLVENT

? 
*** Current Data Parameters $* * *$

NAME : $\quad k m 141$

EXPNO : 4

PROCNO :

***Acquisition Parameters ***

BF1 : $400.1300000 \mathrm{MHz}$

$D[1] \quad: \quad 1.0000000 \mathrm{sec}$

DATE_t : 09:25:50

DATE_d : Nov032006

NS : 16

SOLVENT : $\quad$ CDCl3

SW $\quad: \quad 20.6885 \mathrm{ppm}$

TD : $\quad 65536$

*** Processing Parameters ***

BCFW : 0.0000000

*** 1D NMR Plot Parameters ***

SOLVENT : ?

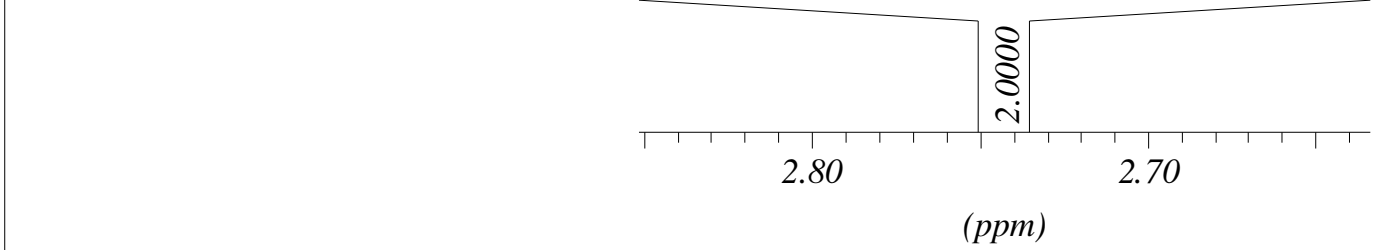

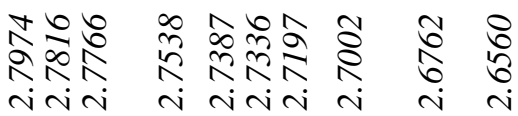

|l |l\} | |

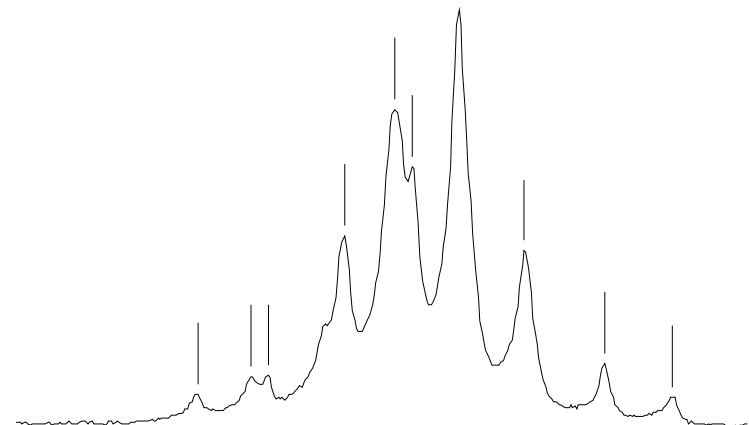

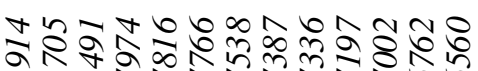

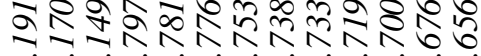

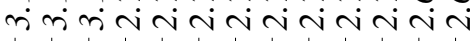

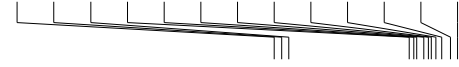

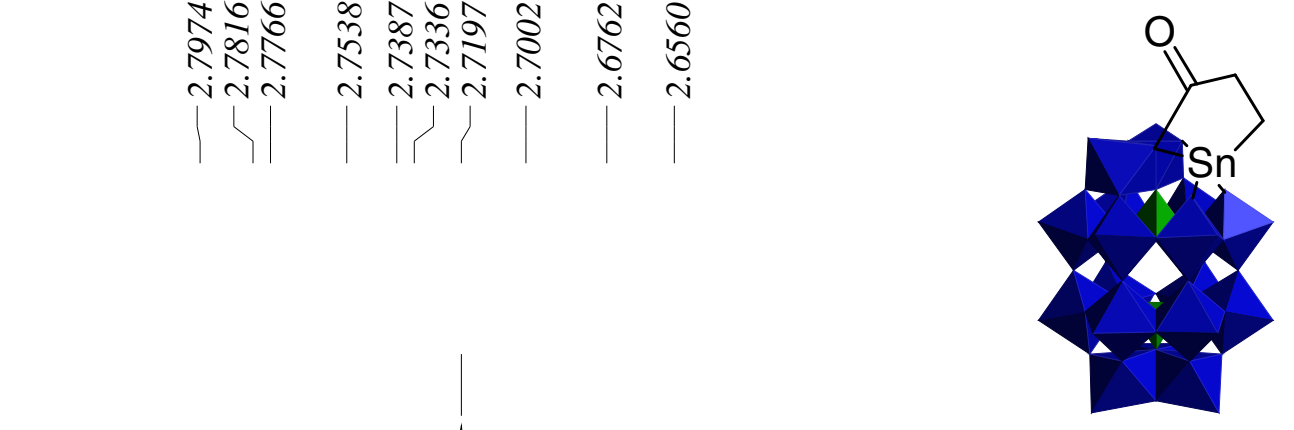

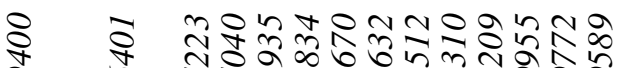

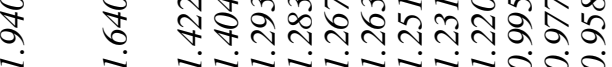




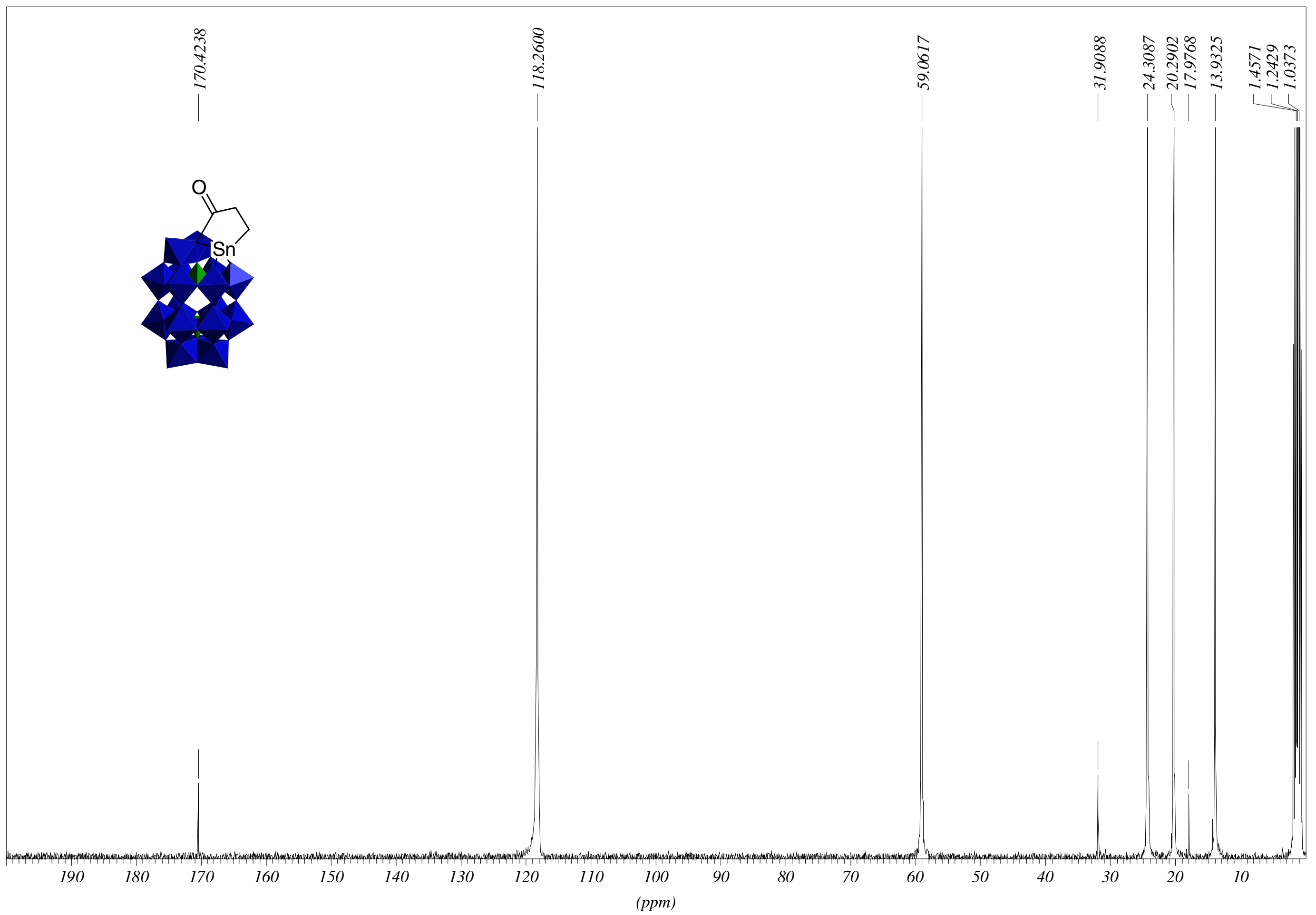


*** Current Data Parameters ***

NAME : $\quad$ 2w17s 1

EXPNO : 5

PROCNO : 1

***Acquisition Parameters ***

BF1 : $111.9203740 \mathrm{MHz}$

$D[1] \quad: 0.1000000 \mathrm{sec}$

$D[33] \quad: 0.0000000 \mathrm{sec}$

DATE_t : $22: 47: 40$

DATE_d : Jul 012007

NS : 1536

SOLVENT : $\quad C D 3 C N$

SW $\quad$ : $44.6952 \mathrm{ppm}$

TD : 16384

*** Processing Parameters ***

BCFW : 0.0000000

*** ID NMR Plot Parameters ***

SOLVENT :

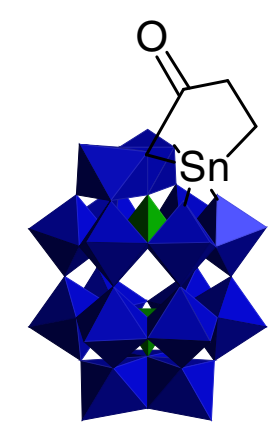

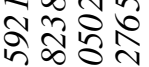

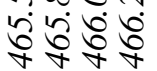

11

11

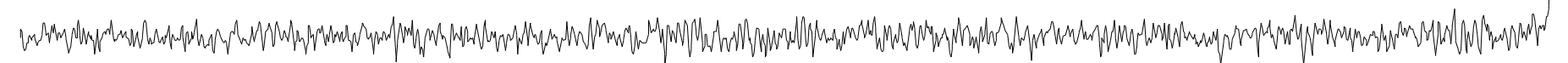

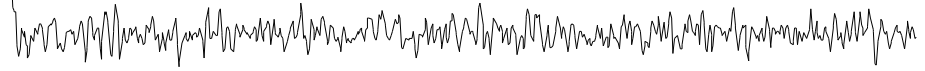




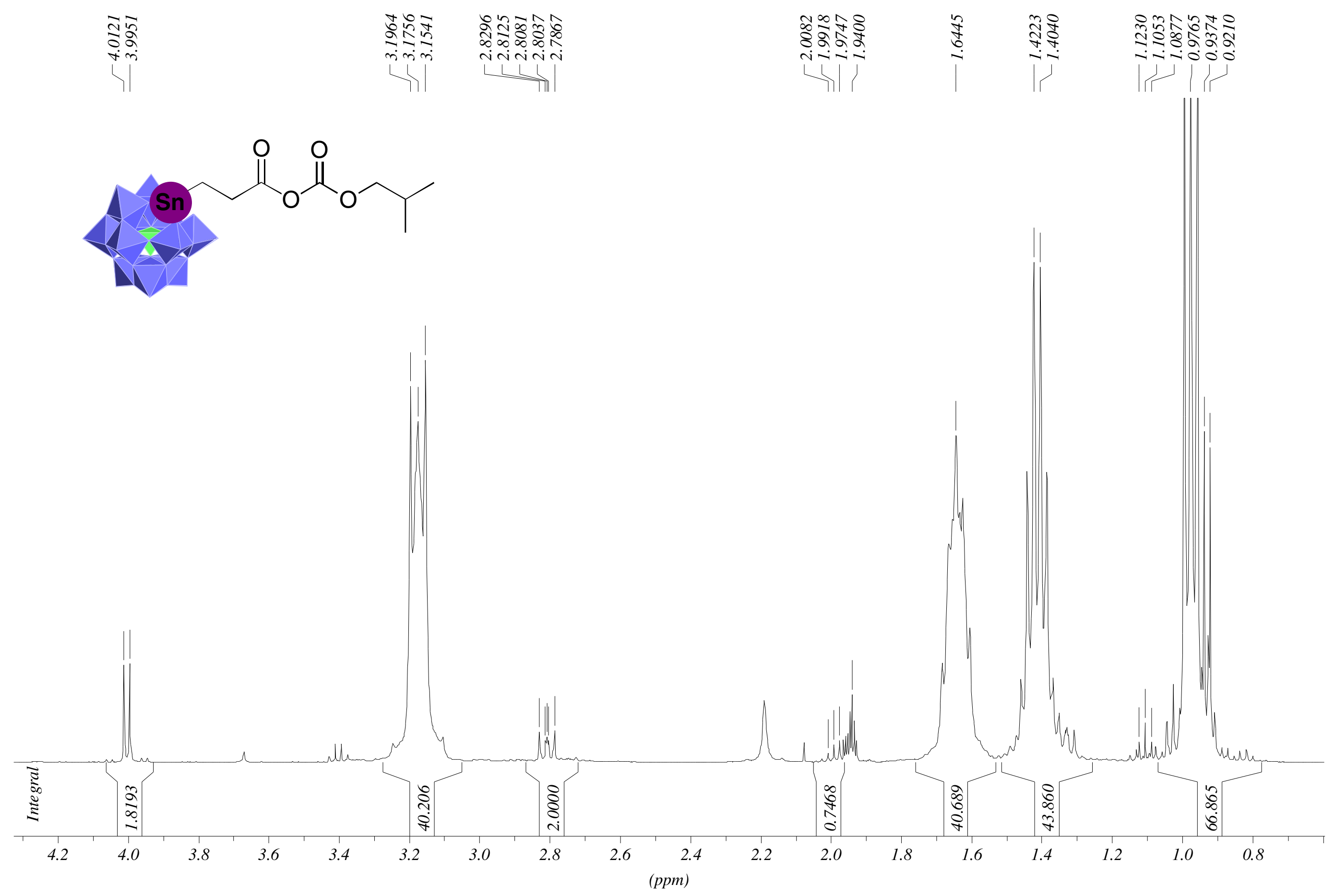




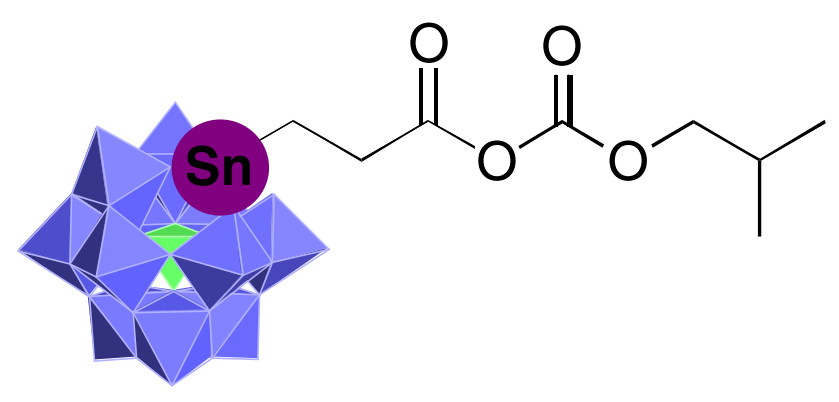

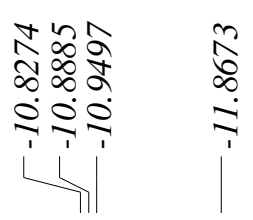

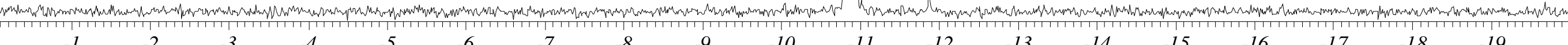
(ppm) 


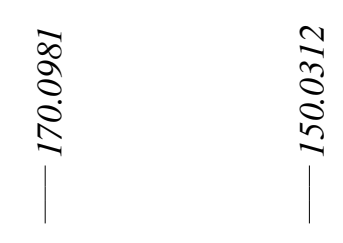

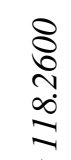

के

$\frac{2}{\frac{2}{2}}$

충ำ

क्ष শंत्र

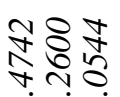

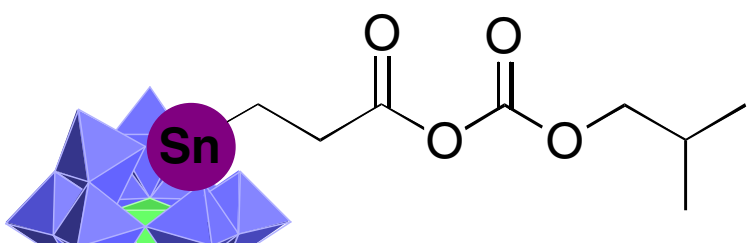


$1 H$

ต

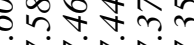

LL L L

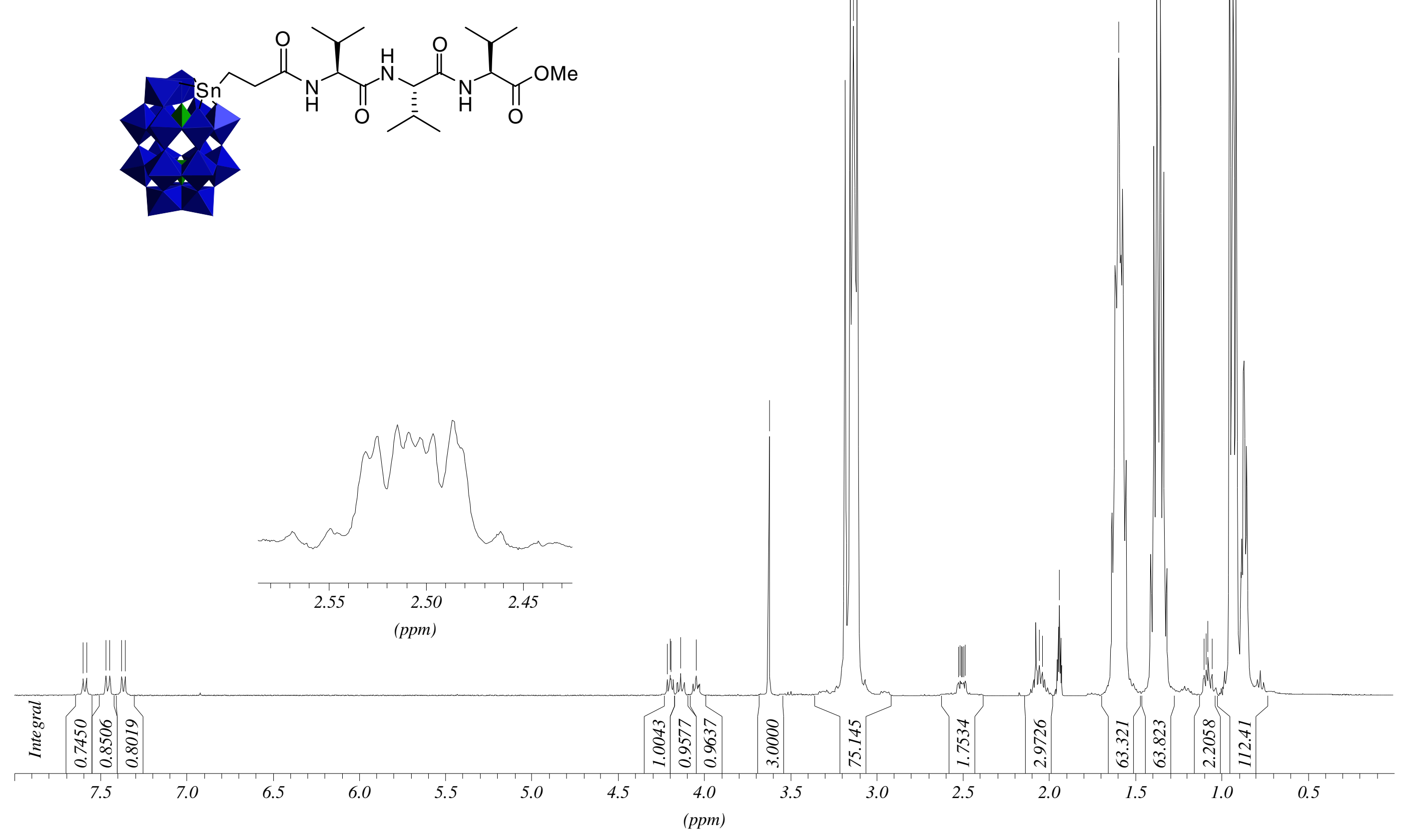

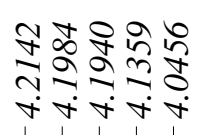

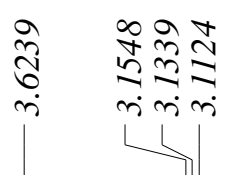

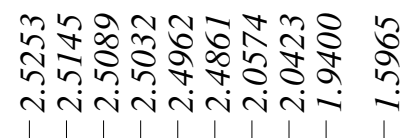

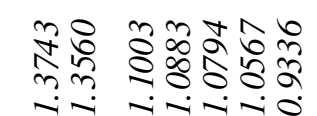


NAME

km359

EXPNO

2

PROCNO

*** Acquisition Parameters ***

BF1 : $161.9755730 \mathrm{MHz}$

$D[1] \quad: \quad 2.0000000 \mathrm{sec}$

DATE_t : 05:21:52

DATE_d : Jul 052007

NS : 32

SOLVENT : $\quad$ CDCl3

SW $\quad$ : $\quad 50.1130 \mathrm{ppm}$

TD : 8190

*** Processing Parameters ***

BCFW : 0.0000000

*** ID NMR Plot Parameters ***

SOLVENT :
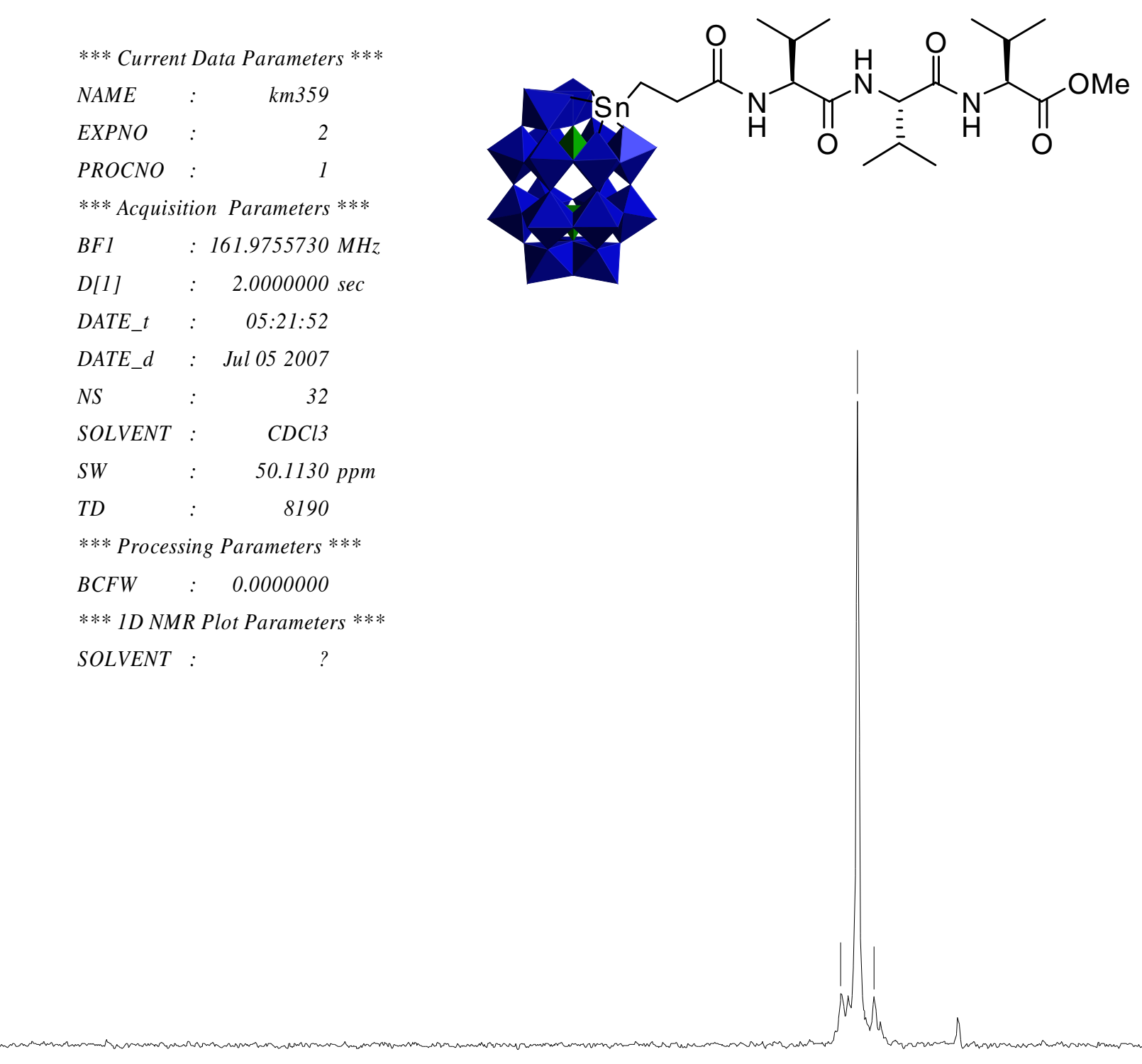


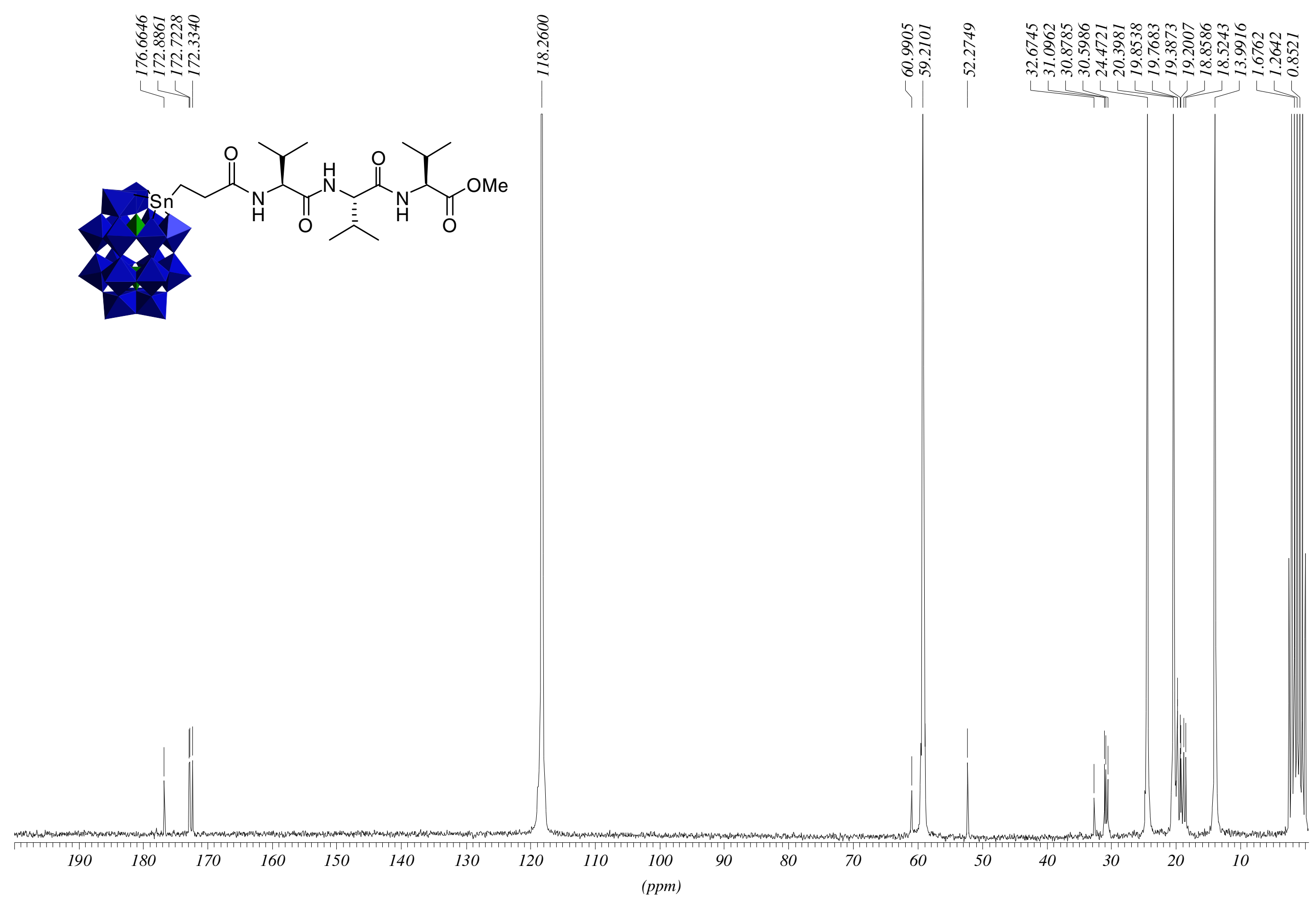




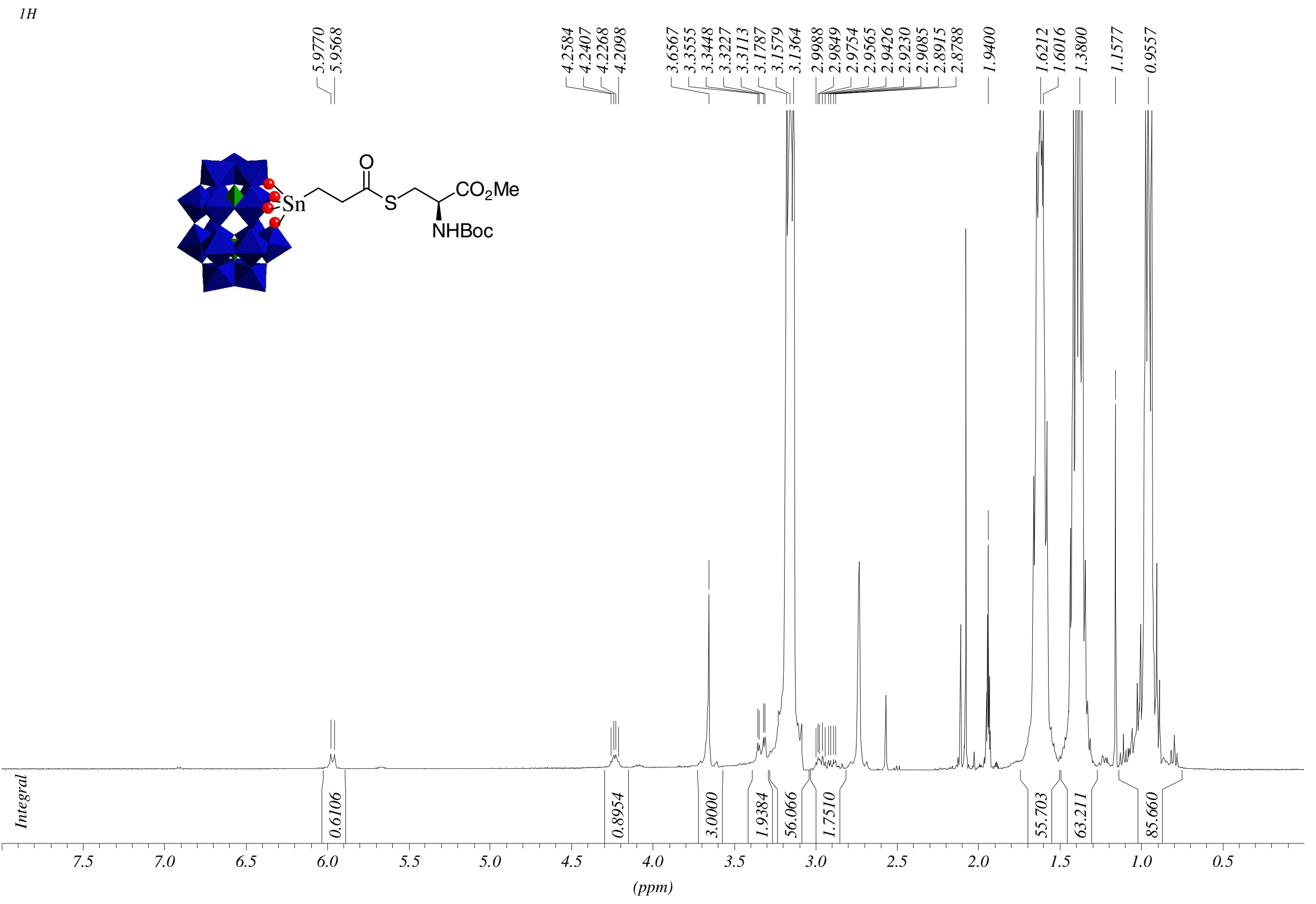




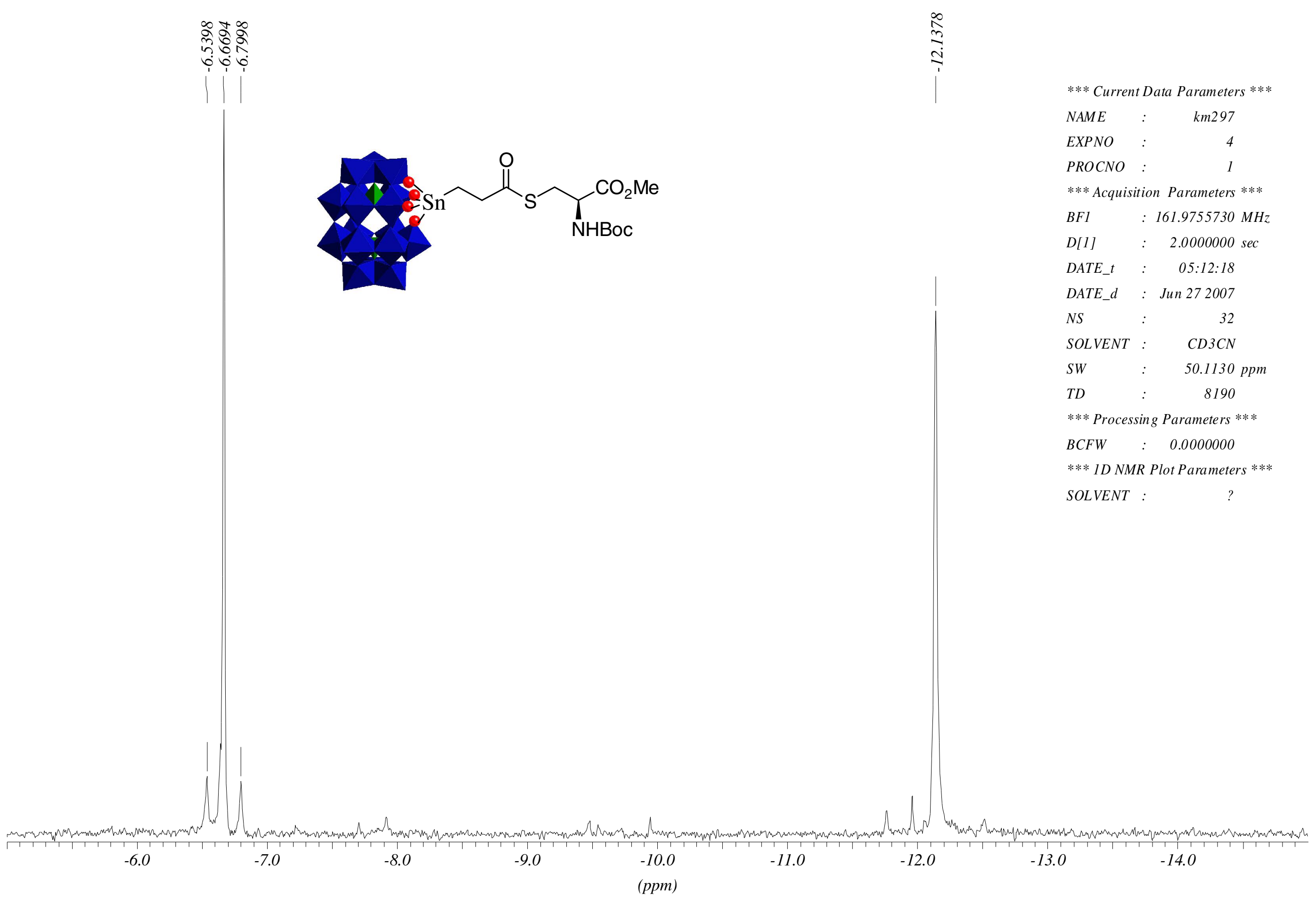




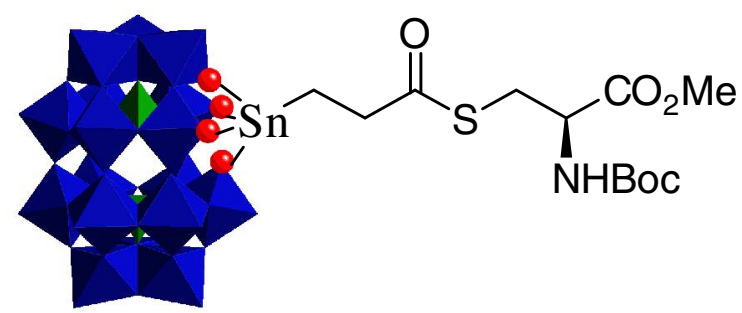




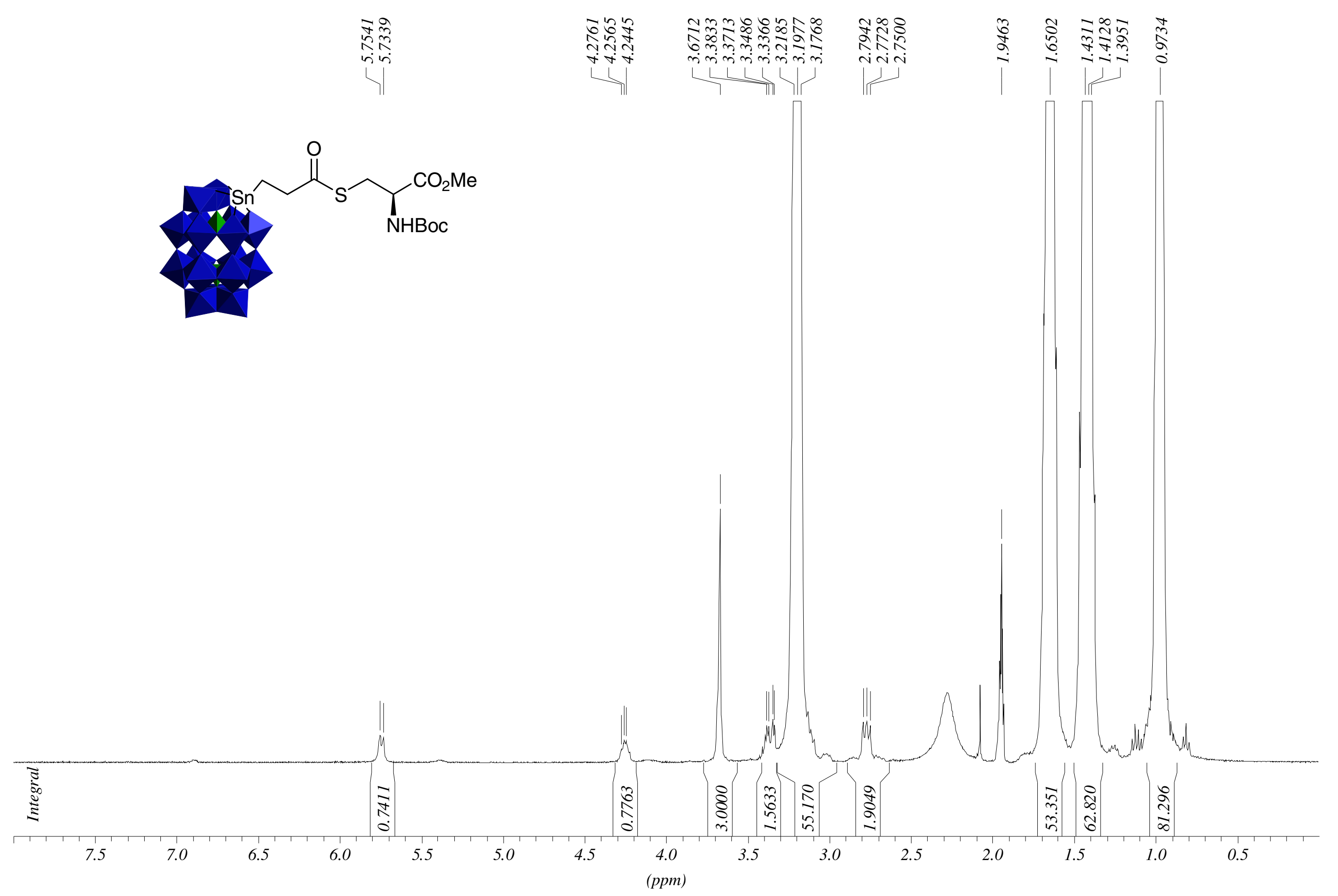




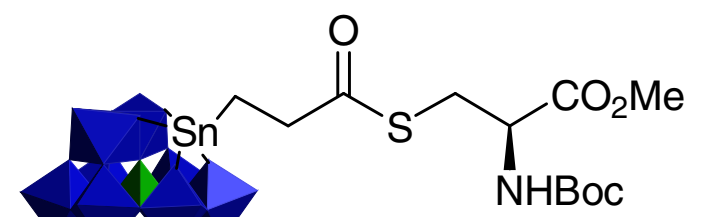

*** Current Data Parameters ***

NAME : $\quad k m 288$

EXPNO

4

PROCNO :

***Acquisition Parameters ***

BF1 : $161.9755730 \mathrm{MHz}$

$D[1] \quad: \quad 2.0000000 \mathrm{sec}$

DATE_t : 05:50:27

DATE_d : Apr 182007

NS : 32

SOLVENT : $\quad C D 3 C N$

SW : $50.1130 \mathrm{ppm}$

TD : 8190

*** Processing Parameters ***

BCFW : 0.0000000

*** 1 D NMR Plot Parameters *** SOLVENT

$?$

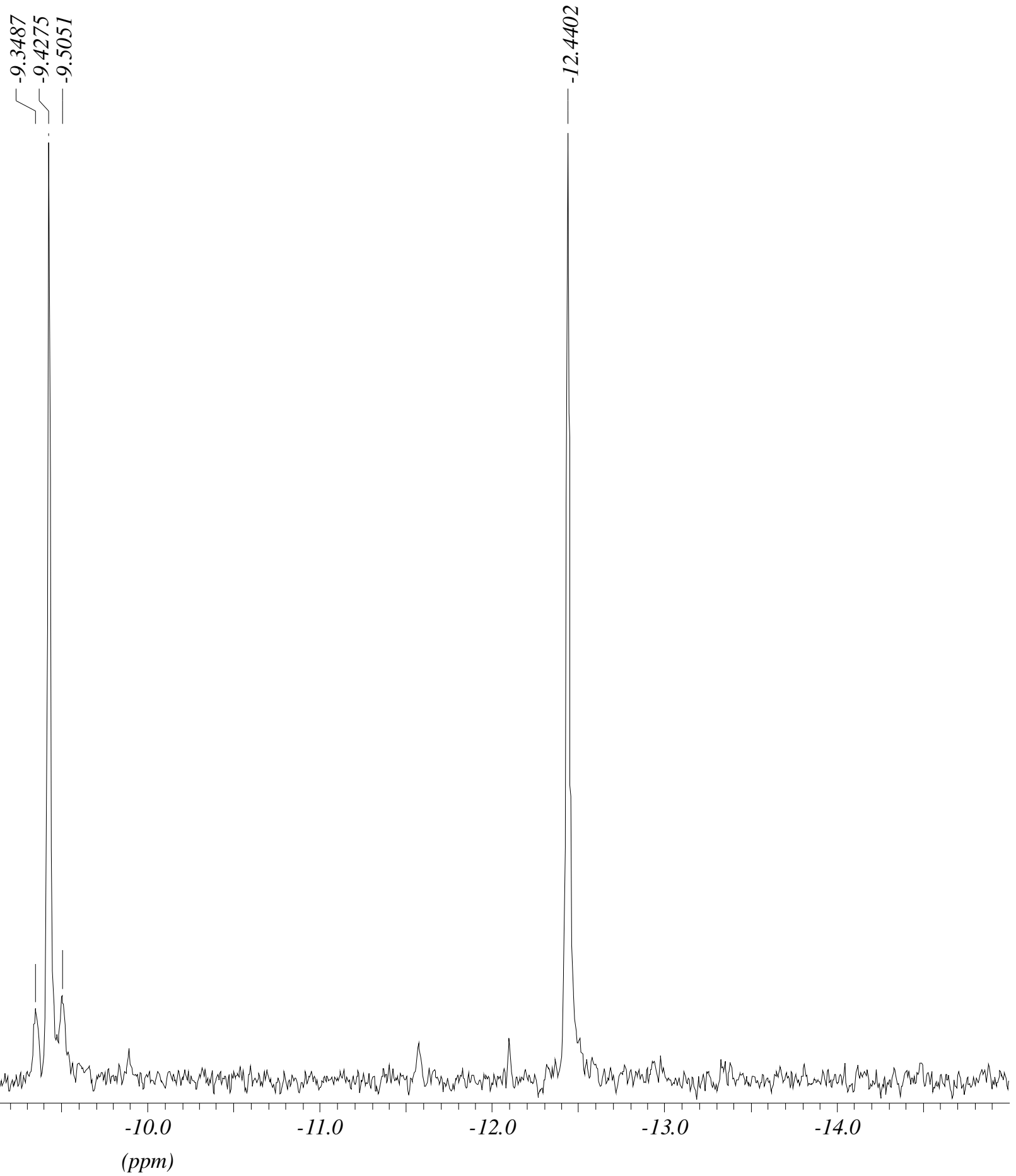




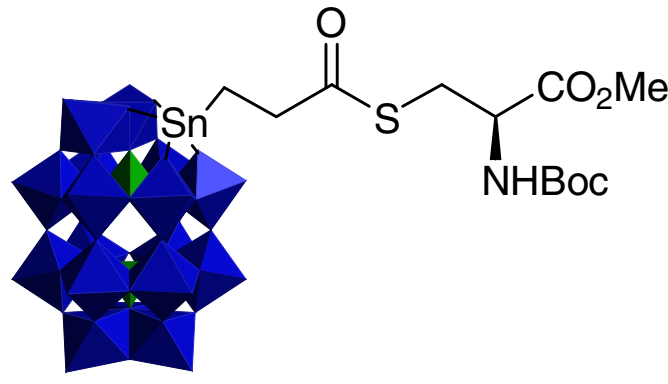

